\title{
Ex-post Evaluation des Strategieprogramms Take Off (2002-2013)
}


Diese Studie wurde im Auftrag des Bundesministeriums für Verkehr, Innovation und Technologie (bmvit) durchgeführt.

VERFASSER/INNEN DES BERICHTS:

Peter Kaufmann (PI)

Christiane Kerlen

Michael Bräuninger (I/O-Analyse)

Laurenz Wolf

INTERNES REVIEW:

Sonja Sheikh

LAYOUT:

Susanne Fröhlich

Die vorliegende Studie wurde nach allen Maßstäben der Sorgfalt erstellt.

Die KMU Forschung Austria übernimmt jedoch keine Haftung für Schäden oder Folgeschäden, die auf diese Studie oder auf mögliche fehlerhafte Angaben zurückgehen.

Dieses Werk ist urheberrechtlich geschützt. Jede Art von Nachdruck, Vervielfältigung, Verbreitung, Wiedergabe, Übersetzung oder Einspeicherung und Verwendung in Datenverarbeitungssystemen, und sei es auch nur auszugsweise, ist nur mit ausdrücklicher Zustimmung des Auftraggebers sowie der KMU Forschung Austria gestattet.

Mitglied bei:

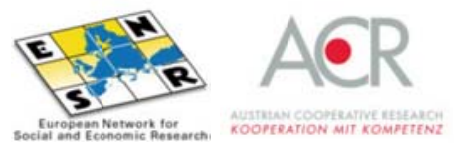




\section{Inhalt}

1. Hintergrund des Programms Take Off ............................................ 1

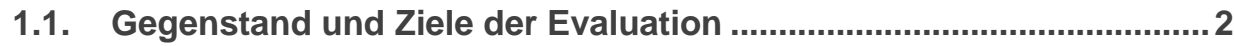

1.2. Evaluationsansatz und Methoden.......................................................

2. Konzeption des Programms …........................................................ 7

2.1. Politischer und institutioneller Kontext.............................................

2.2. Programmziele und deren Kongruenz - Ein Logic Model................. 11

2.3. Einbettung in das restliche FFG Portfolio.........................................16

3. Von Inputs zu Outputs: Aktivitäten und Leistungen des Programms ........................................................................................ 18

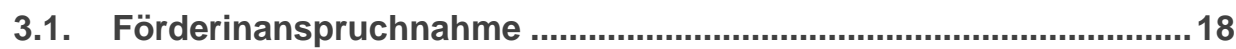

3.2. Sicht der Unternehmen und Forschungseinrichtungen ...................29

3.3. Aufwendungen und Begleitmaßnahmen ............................................40 40

4. Ergebnisse (Outcomes) und Wirkungen (Impacts)...................... 41

4.1. Unmittelbare Ergebnisse bei der Zielgruppe ...................................41

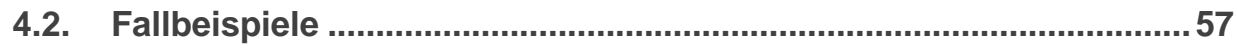

4.3. Gesamtwirtschaftliche Wirkung der Investitionen in Forschung und Entwicklung der Luftfahrtbranche...........................62

5. Schlussfolgerungen und Empfehlungen .................................... 70

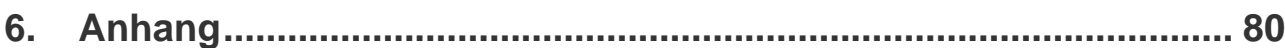

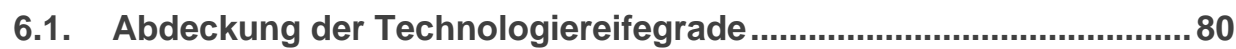

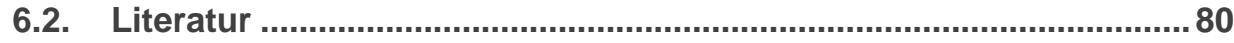

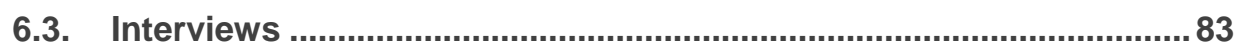




\section{Grafiken}

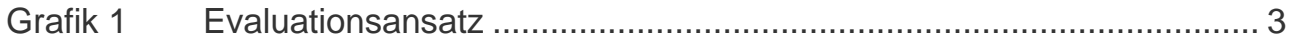

Grafik 2 Analyserahmen und Indikatoren für Take Off ................................... 6

Grafik 3 Entwicklung der Europäischen Luftfahrtstrategie ............................... 7

Grafik $4 \quad$ Logic Model des Programms Take Off............................................. 13

Grafik 5 Take Off Programmbeteiligungen ............................................... 19

Grafik 6 Anteil der Fördersumme bzw. Projektzahl nach Marktsegmenten..... 20

Grafik 7 Take Off Projektbeteiligungen nach Anzahl der Projektträger sowie nach Summe des Barwerts, 2002-13....................................... 21

Grafik 8 Fördernehmer nach Größenklassen und Summe Barwert der Förderung, 2002-13 …....................................................... 22

Grafik 9 Anteil der Projekteilnahmen nach Forschungseinrichtungen, KMU, und Großunternehmen, Ausschreibungen 2002-13................ 23

Grafik 10 Förderbudgets nach Ausschreibung und Organisationstyp der

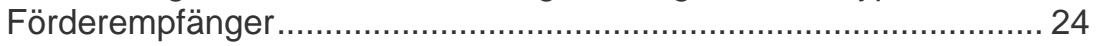

Grafik 11 Regionale Verteilung nach Projektbeteiligungen, 2002-13 ............... 25

Grafik 12 Anzahl von Teilnehmern und Anteil von Erstteilnehmern an

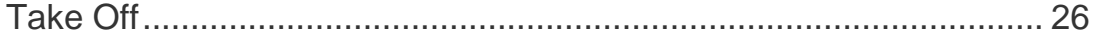

Grafik 13 Kumulierte Zahl der Projektbeteiligungen nach Marktsegmenten ..... 27

Grafik 14 Welche Projektziele wurden verfolgt bzw. erreicht ......................... 30

Grafik 15 Welche Projektziele wurden verfolgt bzw. erreicht ........................... 31

Grafik 16 Programmziele, die durch die Projektergebnisse unterstützt

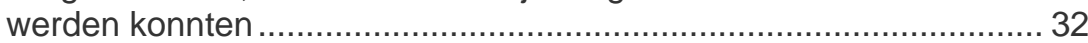

Grafik 17 Programmziele, die durch die Projektergebnisse unterstützt

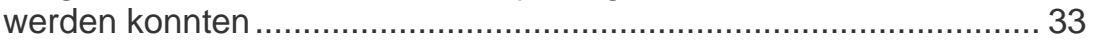

Grafik 18 Technologiereifegrade vor Projektbeginn, angestrebt und

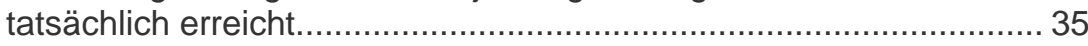

Grafik 19 Technologiereifegrade vor Projektbeginn, angestrebt und

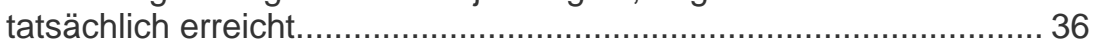

Grafik 20 Differenz zwischen erreichtem TRL und geplantem TRL.................. 37

Grafik 21 Nutzen des Projekts für Unternehmen ............................................ 41

Grafik 22 Nutzen des Projektes für Forschungseinrichtungen ......................... 42

Grafik 23 Differenz zwischen erreichtem TRL und geplantem TRL.................. 43

Grafik 24 Weiterverfolgung der Projekte nach Projektende............................... 44

Grafik 25 Durchführung des Projekts ohne Förderung ...................................... 45

Grafik 26 Weitere Nutzung der technologischen Ergebnisse der Forschungsprojekte .................................................................. 46

Grafik 27 Fortsetzung der Zusammenarbeit mit den Partnern des Kooperationsprojektes nach Ende des Projektes 48 
Grafik 28 Form der Fortsetzung der weiteren Zusammenarbeit ...................... 48

Grafik 29 Stärkere Integration in FuE- und Innovationskooperationen ............... 49

Grafik 30 Marktposition im internationalen Markt der Luftfahrt bzw. der Scientific Community ...................................................................... 50

Grafik 31 Beitrag von Take Off zur Verbesserung der eigenen Marktposition bzw. Position in der Scientific Community .................... 51

Grafik 32 Inanspruchnahme weiterer Förderangebote durch Take Off teilnehmende und nicht-teilnehmende Unternehmen der Luftfahrtzulieferindustrie ........................................................... 52

Grafik 33 Bedeutung von Luftfahrt als Geschäftsfeld......................................53

Grafik 34 Wirtschaftliche Verwertung der Projektergebnisse der Unternehmen 54

Grafik 35 Wirtschaftliche Verwertung der Projektergebnisse der FE.................56

Grafik 36 Förderungen entlang der Technologiereifegrade ............................. 80 


\section{Tabellen}

Tabelle 1 Umweltbezogene und sonstige Ziele der Strategiedokumente VISION 2020 und Flightpath 2050

Tabelle 2 Beteiligung Österreichischer Organisationen an FP6, Luft- und Raumfahrt

Tabelle 3 Rückflussquoten aus FP7 und FP6 sowie European Clean Sky ......... 9

Tabelle 4 Weitere Luftfahrt-Beteiligungen im FFG Portfolio nach Organisationstyp

Tabelle 5 Aktivitäten von Unternehmen zur Verbreitung des Projekts und der Projektergebnisse in der Fachöffentlichkeit ....

Tabelle 6 Aktivitäten der Forschungseinheiten zur Verbreitung des

Projekts und der Projektergebnisse in der Fachöffentlichkeit.

Tabelle 7 Bereits eingetretene ökonomische Effekte aufgrund der Projektergebnisse.

Tabelle 8 Voraussichtlich eintretende ökonomische Effekte aufgrund der Projektergebnisse.

Tabelle 9 Bereits eingetretene ökonomische Effekte aufgrund der Projektergebnisse 56

Tabelle 10 Input-, Verhaltens- und Outputadditionalität der vier Fallstudien ....... 58

Tabelle 11 Investitionen im Luftfahrzeugbau ........................................................ 64

Tabelle 12 Direkte, indirekte und induzierte Effekte der FuE-Ausgaben ............. 66

Tabelle 13 Direkte, indirekte und induzierte Effekte einer Produktneueinführung

Tabelle 14 Direkte, indirekte und induzierte Effekte einer Produktneueinführung in Deutschland 68

Tabelle 15 Multiplikatoren der FuE-Ausgaben in Österreich und in Deutschland. 


\section{Hintergrund des Programms Take Off}

Das Strategieprogramm Take Off wurde im Jahre 2002 aufgrund des folgenden Argumentationsstranges ins Leben gerufen: Erstens hatte sich in Österreich eine kritische Masse an Unternehmen gebildet, die steigende Umsätze in der Luftfahrtbranche erwirtschafteten, sodass eine Unterstützung aus strukturpolitischen Gründen überlegt wurde. Zweitens wurde dieser Branche ein weiteres Wachstum vorausgesagt, und gleichzeitig zeichneten sich mittelfristig einige technologische Umbrüche in dieser und technologisch verwandten Branchen ab (Energie- und Ressourceneinsparung in der Luftfahrt sowie E-Mobilität in der Automobilindustrie). Drittens waren diese Unternehmen in Österreich noch kaum vernetzt und liefen aufgrund dessen Gefahr ihr volles Potential in der auf Systemintegration / Konzentration ausgerichteten Luftfahrtindustrie, mit entsprechenden Konsequenzen für die Zulieferindustrie, nicht ausschöpfen zu können.

Von Ökonomen wird argumentiert, dass Marktversagen in der Luftfahrtindustrie im besonderen Maße zutrifft, da ein sehr wissensintensiver Markt, gekennzeichnet durch Langfristigkeit, Dominanz von wenigen multinationalen Konzernen, sowie die Integration von unterschiedlichen, vertikalen sowie Querschnittstechnologien für die Unternehmen sehr hohe Risiken birgt (Clement und Billovits 2007). Dies wird durch Risikoexperten noch präzisiert: Lange Entwicklungszeiten, die hohe Komplexität von Technologien und damit Projekten sowie der daraus resultierenden Finanzierungsund Investitionsbedarf resultiert in überdurchschnittlich hohe Risiken verschiedener Art (Forstinger et al. 2014).

Da somit konjunkturelle und insbesondere technologische Einflüsse eine Hebelwirkung auf einen immer größeren Teil der österreichischen Beschäftigten ausüben konnte, wurde mit dem Strategieprogramm Take Off eine weitere Diversifizierung in einen Wachstumsmarkt getätigt. Mit dem Programm wurde es auch leichter möglich, im Rahmen der EU Initiative ERA NET aktiv zu werden. Österreich war bis 31.12.2013 im Rahmen von ERA NET AirTN eines von 18 Partnerländern, wobei mit dem deutschen Koordinator DLR besonders eng zusammengearbeitet wurde. Derzeit ist AirTN eine CSA (Coordination and Support Action) unter italienischer Koordination mit 10 Partnerländern. Ziel ist die Konzentration auf gemeinsame Ausschreibungen, die Förderung gemeinsamer transnationaler innovativer Projekte sowie die weitere Vernetzung des AirTN Konsortiums.

Seit dem Jahr 2008 stellt die FTI-Luftfahrtstrategie den Rahmen für das Programm Take Off dar, in der die Trends in der Luftfahrt sowie die Potenziale für die österreichische Luftfahrtindustrie abgesteckt, und in erfreulicher Konkretheit die angestrebten Maßnahmen definiert wurden. 2014 erfolgte eine Neuauflage der FTI-Strategie. 


\subsection{Gegenstand und Ziele der Evaluation}

Gegenstand der Evaluation ist das österreichische Luftfahrtprogramm Take Off, mit dem im Zeitraum 2002-2013 Mittel in Höhe von € 61,2 Mio. (inkl. vier ERA-Net AirTN Projekte) vorwiegend für kooperative Forschungs- und Entwicklungsprojekte bereitgestellt wurden.

Gemäß dem Take Off Programmdokument waren neben den Programmwirkungen auch die Positionierung des Programms in der österreichischen Förderungslandschaft unter dem Aspekt der Optimierung des Förderungsportfolios zu thematisieren. Damit zielt die Evaluierung auf eine möglichst umfassende Wirkungserfassung und die Darstellung der Forschungsförderung im Themenbereich der Luftfahrt ab. Entlang der gesellschafts- und technologiepolitischen Schwerpunktsetzung von Take Off erfolgt eine Beurteilung dahingehend, ob die eingesetzten, operationalen Zielsetzungen mit Hilfe der hinzugezogenen Instrumente erreicht werden konnten (Designanalyse). Darüber hinaus wird der Frage nachgegangen, in welchem Ausmaß die gesetzten Programmziele auch tatsächlich erreicht werden konnten und welche direkten sowie indirekten Effekte sich auf die Forschungsförderung zurückführen lassen (Wirkungsanalyse). Mit der Evaluierung sollen zum einen Informationen über die Programmgenese und Programmwirkungen für die fachkundige Öffentlichkeit generiert werden, und zum andern sollen dem BMVIT und der FFG relevante Informationen für eine etwaige Weiterführung sowie zukünftige Gestaltung des Programms Take Off zur Verfügung gestellt werden. 


\subsection{Evaluationsansatz und Methoden}

Die im folgenden Bericht dokumentierten Aussagen hinsichtlich der Effektivität der Unterstützungsmaßnahmen und einem möglichen Optimierungsbedarf in der Programmausgestaltung fußen auf dem Einsatz geeigneter quantitativer und qualitativer Methoden. Der Evaluationsansatz teilt sich dabei auf die folgenden Arbeitsschritte und Methodenanwendungen auf:

- Analyse von Literatur, Monitoringdaten und Interviews (Auswertungen verfügbarer Studien, Fachliteratur, FFG-Projektdaten, Fachinterviews)

- Wirkungsmodell (auf den Ergebnissen aufbauende Erfassung und Darstellung von programmintendierter Wirkungsmechanismen)

- Befragung (von Unternehmen und Forschungseinheiten mittells OnlineFragebogen)

- Fallstudien (Detailbetrachtung von Wirkungsverläufen ausgewählter Forschungsprojekte bzw. Projektketten)

- I/O-Analyse (Quantifizierung erzielter Wirkungen auf gesamtwirtschaftlicher Ebene)

- Reflexionsworkshop (Präsentation und Diskussion der Ergebnisse und Handlungsempfehlungen)

Grafik 1 Evaluationsansatz

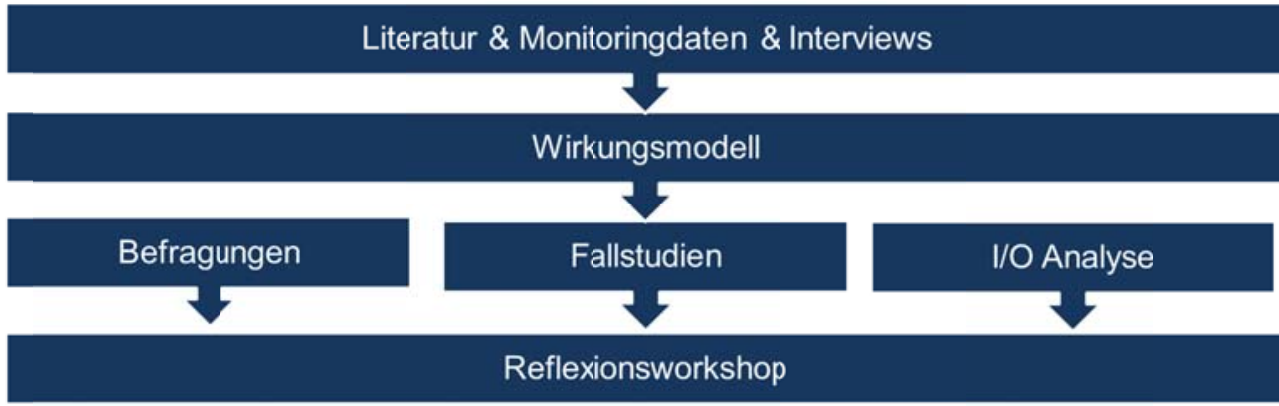

Quelle: KMU Forschung Austria

\section{Literatur \& Monitoringdaten \& Interviews}

Die Berücksichtigung und Auswertung vorhandener Literatur aus dem Themenfeld der Luftfahrt beinhaltet die bereits abgeschlossene Zwischen- und Endevaluierungen (TAKE OFF und LUFO), Programm- und Strategiedokumente, Projektberichte, sowie Präsentationen und Veranstaltungsprotokolle. Die daraus gewonnenen Erkenntnisse fokussierten die weitere Vorgehensweise, ermöglichten das Aufgreifen vielversprechender Wirkungsanalysen, das Vermeiden redundanter Analyseschritte und das Anknüpfen an relevante Zwischenergebnisse. 
Daneben bildete die Auswertung der Monitoringdaten den Ausgangspunkt für einen umfassenden Überblick der abgewickelten Forschungsprojekte und zur Erfassung der vorliegenden Fördernehmerstrukturen.

Schließlich wurden bereits im ersten Arbeitsschritt auch 10 Experteninterviews durchgeführt, welche die organisatorische Programmstruktur und Programmabwicklung erfassten. Dabei wurden auch Gespräche mit Verantwortlichen von vergleichbaren, ausländischen Institutionen und Unterstützungsprogrammen durchgeführt, um die Erkenntnisse dieser Evaluation in einen internationalen Kontext zu setzen.

\section{Wirkungsmodell}

Das Wirkungsmodell dient dazu, die Funktionsweise und die konzeptionellen Wirkzusammenhänge des Strategieprogramms Take Off in strukturierter und vereinfachter Form darzustellen. Mission, Programmziele, Inputs und Aktivitäten sowie die erzielten Leistungen und Ergebnisse auf den Ebenen Outputs, Outcomes und Impacts wurden dazu in einem Logic Chart dargestellt, und einer kritischen Analyse unterzogen.

\section{Online-Befragungen}

Auf die oben erwähnten Analyseschritte folgte eine Online-Befragung der involvierten Unternehmen und Forschungseinrichtungen (FE) zu den Effekten der Take Off Projekte. Diese wurde anhand zweier unterschiedlicher Fragebögen umgesetzt. Der Befragungszeitraum erstreckte sich von Ende Juli bis Mitte September 2014, wobei insgesamt drei Aufforderungen zur Teilnahme versendet wurden.

Adressaten der Befragung waren 104 Fördernehmer (69 Unternehmen und $35 \mathrm{FE}$ ) bzw. 206 Förderfälle, die aus der Bereinigung der 309 Projektteilnahmen mit bereits abgeschlossenen Projekten resultierten. Nicht einbezogen wurden Projekte und Projektteilnehmer aus dem Bereich Human Resources und den Begleitmaßnahmen, ausländische Projektteilnehmer, Projekte mit einem Fördervolumen von weniger als $€ 20.000$ sowie Fördernehmer in Liquidation. Die verbliebenen 206 Projektteilnahmen konzentrierten sich auf 81 Projekte. Darüber hinaus wurden die Fördernehmer aufgefordert, Projektketten innerhalb eines Fragebogens abzuhandeln. Im Laufe der Befragung gingen 68 vollständig ausgefüllte Fragebögen ein, die sich auf 46 Projekte und 86 Projektteilnahmen bezogen. Jeweils 34 der Fragebögen sind den Unternehmen bzw. den Forschungseinrichtungen zuzurechnen. Dies resultiert in einen Rücklauf von $32 \%$ bei den Unternehmen sowie $51 \%$ bei den FE. Während bei den FE von einem repräsentativen Rücklauf ausgegangen werden kann, ist dies bei den Unternehmen insofern eingeschränkt, als Unternehmen mit technologischen Projekten und Projektende Mitte 2014 überproportional größere Unternehmen beinhaltet (d.h. ohne Begleitmaßnahmen, und Unternehmen, die bereits seit längerem in der

Luftfahrt tätig sind). 
Zeitgleich zur Take Off Evaluierung wurde von Brimatech Services GmbH im Rahmen des bmvit Projektes Ö-LINKupdate eine Befragung der österreichischen Luftfahrt(zuliefer)industrie durchgeführt. Um den Aufwand für die Unternehmen zu minimieren, wurden die Befragungen zeitlich aufeinander abgestimmt und der Fragebogen der Evaluierung gekürzt. Damit wurde eine mehrmalige Erhebung derselben bzw. ähnlicher Daten vermieden; Brimatech erklärte sich bereit, der KMU Forschung Austria einige Ergebnisse aus dem Ö-LINKupdate zur Verfügung zu stellen. Zur Wahrung der Anonymität wurden ausschließlich anonymisierte und kumulierte Daten weitergegeben.

\section{Fallstudien}

Die Durchführung von Fallstudien diente in weiterer Folge der detaillierten Betrachtung einzelner Projekte bzw. Projektketten. Dabei erfolgte die Auswahl der zu betrachtenden Forschungsvorhaben in enger Abstimmung mit dem bmvit/FFG und zielte auch auf die im Rahmen der Technologieplattform Offset initiierten Projekte ab. Insgesamt wurden vier Fallstudien erstellt, wobei sich diese hinsichtlich der Projektcharakteristik klar unterschieden (Themenfelder, Zahl und Art der Projektteilnehmer, Projektdauer und Umfang, technologisches Ausgangsniveau).

Aufgrund des hohen Detailgehaltes der Fallstudien, und der daraus resultierenden Sensitivität der (Unternehmens-)Information sind die Fallstudien in diesen Bericht in gekürzter Form inkludiert.

\section{Input-Output-Analyse}

Die I/O-Analyse als Methode der empirischen Wirtschaftsforschung ermöglicht es, Zusammenhänge unterschiedlicher Wirtschaftssektoren abzubilden und nicht mittelbare Programmwirkungen zu erfassen. Dadurch lässt sich die Wirkungsbreite der Take Off Förderungen von der Projekt- bzw. Programmebene auf die volkswirtschaftliche Ebene heben und Aussagen zu gesamtwirtschaftlichen Effekten abschätzen. Mit Hilfe von Input-Output-Tabellen der Statistik Austria, die unter der Annahme konstanter, linearer Lieferbeziehungen zwischen den Wirtschaftssektoren erstellt werden, lassen sich Auswirkungen von Änderungen der Endnachfrage ableiten und schließlich auch quantifizieren. Die Quantifizierung der Programmeffekte ergibt sich dabei aus der Summe der direkten (bei der Beteiligung anfallenden Wertschöpfung) und den indirekten Effekten (Vorleistungen entlang der Wertschöpfungskette). Zur leichteren Einordnung der Ergebnisse werden die Resultate mit den Ergebnissen der I/O-Analyse der deutschen Luftfahrtforschung verglichen, die mit derselben Methodik (und denselben Personen) ermittelt wurden. Daher ist ein Vergleich dieser Werte zulässig. 


\section{Indikatoren}

Für alle im Rahmen der Evaluation betrachteten Analyseebenen wurden geeignete Indikatoren entwickelt. Diese sind in der folgenden Grafik im Überblick wiedergegeben.

\section{Grafik 2 Analyserahmen und Indikatoren für Take Off}

\begin{tabular}{|c|c|c|c|c|}
\hline \multicolumn{5}{|c|}{ Programmdesign } \\
\hline Input & Aktivitäten & Output & Outcome & Impact \\
\hline $\begin{array}{l}\text { - Finanzielle Projektmittel } \\
\text { (öffentliche) } \\
\text { - Finanzielle Projektmittel } \\
\text { (aus den Unternehmen) } \\
\text { - Input-Additionalität: } \\
\text { durch öffentliche } \\
\text { Förderung ausgelöstes } \\
\text { privates FuE-Investment } \\
\text { - Mittel und Kompetenzen } \\
\text { für die Programn- } \\
\text { begleitung }\end{array}$ & $\begin{array}{l}\text { - Nutzung der FFG } \\
\text { Instrumente durch } \\
\text { Fördernehmer } \\
\text { - Organisation von } \\
\text { Vernetzungs- } \\
\text { veranstaltungen } \\
\text { - Teilnahme an Strategie- } \\
\text { und Abstimmungs- } \\
\text { prozessen } \\
\text { - Kooperation mit anderen } \\
\text { Forschungsprogrammen }\end{array}$ & $\begin{array}{l}\text { - Bedeutung einzelner } \\
\text { Projektziele und } \\
\text { Zielerreichungsgrad } \\
\text { (Technologsche Ziele, } \\
\text { Ziele in Bezug auf } \\
\text { Forschung, } \\
\text { betriebswirschaftliche } \\
\text { Ziele, Kooperationsziele) } \\
\text { - Weiterentwicklung von } \\
\text { Technologien (TRL) } \\
\text { - Verbreitung/ Transfer der } \\
\text { Projektergebnisse } \\
\text { - Anmeldung gewerblicher } \\
\text { Schutzrechte } \\
\text { - Qualifizierung (Trainings, } \\
\text { Dissertationen, Studien- } \\
\text { abschlussarbeiten) } \\
\text { - Abdeckung der } \\
\text { Marktsegmente MS1-8 } \\
\text { - Struktur und Umfang der } \\
\text { Vernetzung neue } \\
\text { Kooperationen zwischen } \\
\text { Unternehmen und/oder } \\
\text { Forschungseinheiten }\end{array}$ & 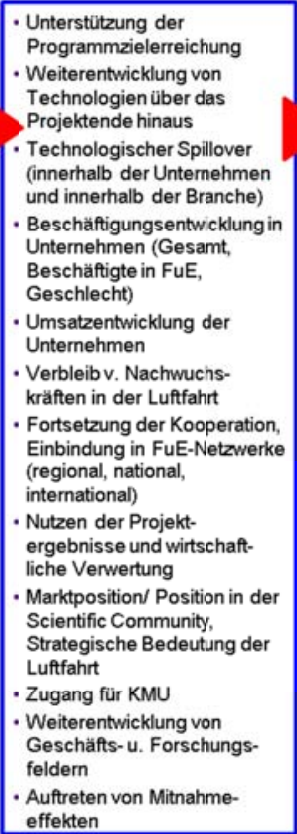 & $\begin{array}{l}\text { - Wertschöpfung und } \\
\text { Beschäftigung in der } \\
\text { osterreichischen Lufffahrt } \\
\text { und Lufffahrtforschung } \\
\text { Verbleib qualifizierter } \\
\text { Fachkräfte in Österreich } \\
\text { - Stärkere internationale } \\
\text { Sichtbarkeit } \\
\text { - Steigerung der } \\
\text { Innovationsfähigkeit der } \\
\text { Branche } \\
\text { - Technologischer Spillover } \\
\text { in andere Branchen }\end{array}$ \\
\hline Kontext & & & In 2020 \& EC Flightpat & \\
\hline
\end{tabular}

Quelle: KMU Forschung Austria und Partner auf Basis von Programmdokumenten und Interviews

\section{Reflexionsworkshop}

Die Evaluationsergebnisse wurden schließlich im Zuge eines Reflexionsworkshops präsentiert und daraus abgeleitete Handlungsempfehlungen vorgeschlagen. Teilnehmer des Workshops waren Mag. Evelinde Grassegger und Dipl.-Ing ${ }^{\text {in }}$. (FH) Ingrid Kernstock vom BMVIT, Dipl.-Ing ${ }^{\text {in }}$. (FH) Vera Eichberger und Daniel Jokovic MSc von der FFG sowie Mag. Peter Kaufmann, Dr. Christiane Kerlen, Dr. Sonja Sheikh und Laurenz Wolf vom Evaluationsteam. 


\section{Konzeption des Programms}

\subsection{Politischer und institutioneller Kontext}

\section{Europäische Luftfahrtstrategie und -förderung}

Die heutige europäische Strategie zur Entwicklung des Luftverkehrs und der Luftfahrtindustrie geht auf eine im Jahr 2000 in Lissabon unterschriebene Erklärung führender europäischer Persönlichkeiten des Luftfahrtsektors („Group of Personalities") zurück. Diese wurde vom damaligen EU-Forschungskommissar Phillippe Busquin beauftragt, eine Vision für die europäische Luftfahrt im Jahr 2020 zu entwickeln („European Aeronautics: A Vision for 2020“). Damit wurde ein Prozess zur Entwicklung einer gemeinsamen Forschungsagenda für den Luftfahrtsektor initiiert und es formte sich der europäische Luftfahrtforschungsbeirat ACARE (Advisory Council for Aeronautics Research in Europe), in dem wichtige Stakeholder der Luftfahrt wie Luftfahrtindustrie, Flughafenbetreiber, Luftverkehrsgesellschaften, Behörden und die Wissenschaft zusammen arbeiten.

Vorrangige Ziele waren und sind, die Technologie- und Marktführerschaft der europäischen Luftfahrt zu erreichen sowie die gesellschaftlichen Anforderungen an einen modernen Luftverkehr zu erfüllen. In der VISION 2020 wurden u.a. ehrgeizige Ziele zur Entlastung der Umwelt gesetzt und die Empfehlung ausgesprochen, eine strategisch ausgerichtete Forschungsagenda zu formulieren, um diese erreichen zu können. In den Jahren 2002, 2004 und 2008 wurden mit der „Strategic Research Agenda 1", der „Strategic Research Agenda 2" und dem „Addendum“ Maßnahmen definiert, weiterentwickelt und konkretisiert.

\section{Grafik 3 Entwicklung der Europäischen Luftfahrtstrategie}

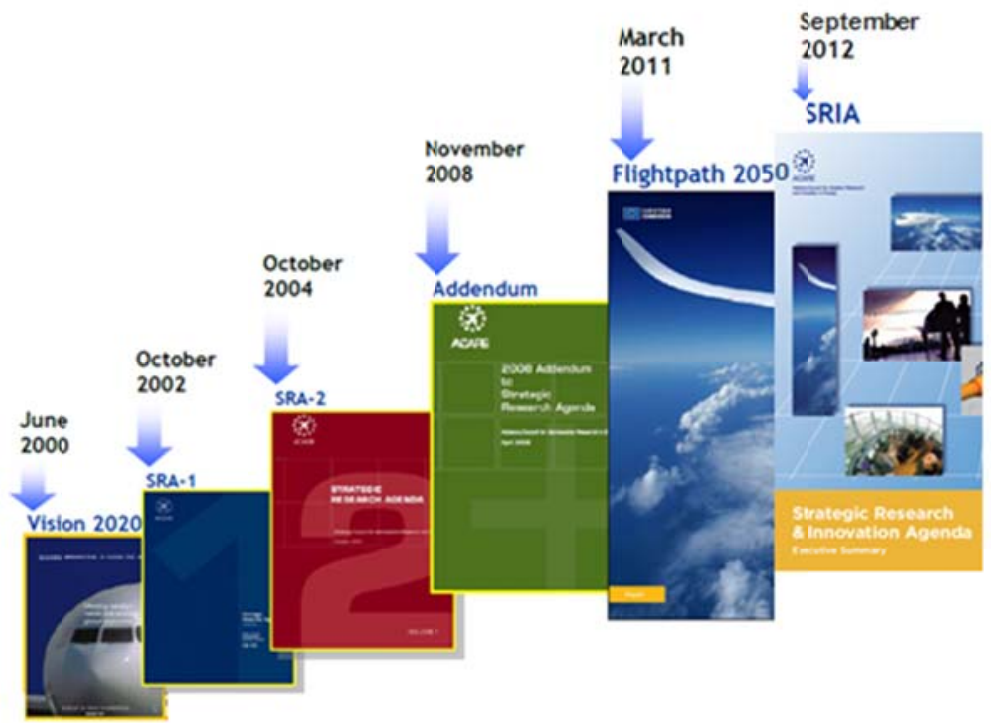

Quelle: in Anlehnung an Knörzer 2012 
Mit Flightpath 2050 wurde im Jahr 2011 die VISION 2020 aktualisiert. Die Europäische Kommission (2011) plädiert in „Flightpath 2050 - Europe's Vision for Aviation“ insbesondere dafür, der europäischen Luftfahrtindustrie zu einer weltweiten Führungsstellung zu verhelfen und die Wettbewerbsfähigkeit, Umweltfreundlichkeit und Sicherheit der Luftfahrt zu fördern, wobei die Bedürfnisse der Gesellschaft und der Bürger in den Mittelpunkt der Strategie zu stellen sind.

Im Herbst 2012 wurde auf der Internationalen Luftfahrtausstellung ILA in Berlin von ACARE die "Strategic Research and Innovation Agenda (SRIA)" vorgestellt, die die Umsetzung von Flightpath 2050 konkretisiert. Die folgende Tabelle fasst die wichtigsten Zielgrößen der beiden Strategiedokumente zusammen.

Tabelle 1 Umweltbezogene und sonstige Ziele der Strategiedokumente VISION 2020 (ACARE) und Flightpath 2050 (Europäischen Kommission) ${ }^{1}$

\begin{tabular}{lcc}
\hline & VISION 2020 & \multicolumn{1}{c}{ FLIGHTPATH 2050 } \\
\hline $\mathrm{CO}_{2 .}$ & $-50 \%$ & $-75 \%$ \\
\hline $\mathrm{NOx}$ & $-80 \%$ & $-90 \%$ \\
\hline Lärm & $-50 \%$ & $-65 \%$ \\
\hline Sonstige Ziele & $\begin{array}{l}99 \% \text { der Flüge starten und landen mit weniger als 15 Min. } \\
\text { Verspătung } \\
\text { Fünffache Reduzierung der durchschnittlichen Unfallrate } \\
\text { bei global operierenden, kommerziellen Flugzeugbetreibern }\end{array}$ & $\begin{array}{l}\text { Reisezeit von Haustür zu Haustür innerhalb Eurcpas ist für } \\
90 \% \text { der Reisenden in 4h möglich } \\
\text { Verbesserung der Sicherheit: u. a. weniger als 1 Unfall bei } \\
10 \text { Millionen Flügen }\end{array}$ \\
\hline
\end{tabular}

Quelle: BMWi 2014, S. 11

Die Umsetzung der europäischen Luftfahrtstrategie erfolgt im Rahmen der Technologie- und Forschungsprogramme auf europäischer Ebene und ist in den Luftfahrtstrategien einzelner Mitgliedstaaten verankert, so auch in der österreichischen FTI-Luftfahrtstrategie.

Das 6. Forschungsrahmenprogramm (Laufzeit Juni 2002 bis 2006) hatte ein Budget von 17,5 Mrd. Euro, von denen rund 1 Mrd. Euro (5,7 \%) auf die thematische Priorität Luft- und Raumfahrt entfielen. Die Prioritäten der Forschungs- und Entwicklungsaktivitäten im Luftfahrtbereich lagen in diesem Zeitraum bei der Verminderung der Umweltauswirkungen in Form von Abgas- und Lärmemissionen der Triebwerke und bei der Steigerung von Kapazität und Betriebssicherheit des Luftverkehrsmanagementsystems, um den europäischen Luftraum zu vereinheitlichen.

Auch wenn die Zahl der Beteiligungen österreichischer Organisationen mit 36 noch relativ gering ausfiel, stellte es eine Steigerung gegenüber der Beteiligung am 4. und 5. Forschungsrahmenprogramm dar (4 bzw. 22) (FFG 2007).

\footnotetext{
${ }^{1}$ Referenzpunkt ist jeweils ein typisches Neuflugzeug im Jahr 2000.
} 
Tabelle 2 Beteiligung Österreichischer Organisationen an FP6, Luft- und Raumfahrt

\begin{tabular}{|c|c|c|c|c|c|c|c|}
\hline \multirow{2}{*}{$\begin{array}{l}\text { Statistischer } \\
\text { Öberblick }\end{array}$} & \multicolumn{3}{|c|}{ Gesamt } & \multicolumn{3}{|c|}{ Österreich } & \multirow{2}{*}{$\begin{array}{c}\text { Anteile } \\
\text { bewilligt } \\
\text { Österreich } \\
\text { an bewilligt } \\
\text { Gesamt }\end{array}$} \\
\hline & evaluiert & bewilligt & $B Q$ & evaluiert & bewilligt & BQ & \\
\hline Projekte & 603 & 192 & $32 \%$ & 86 & 30 & $35 \%$ & $15,6 \%$ \\
\hline Beteiligungen & 7.382 & 2.793 & $38 \%$ & 108 & 36 & $33 \%$ & $1,3 \%$ \\
\hline KoordinatorInnen & 603 & 192 & $32 \%$ & 7 & 2 & $29 \%$ & $1,0 \%$ \\
\hline
\end{tabular}

$\mathrm{BQ}$ : Bewilligungsquote

Quelle: FFG 2007, S. 20

Das 7. Forschungsrahmenprogramm der Europäischen Union belief sich auf 50,5 Mrd. Euro für die Gesamtlaufzeit 2007 bis 2013. Für den thematischen Bereich Verkehr (einschließlich Luftfahrt) standen 4.160 Mio. Euro (8,2 \%) zur Verfügung. Die Prioritäten bezogen sich im Bereich Luftfahrt auf die Themen umweltschonender Luftverkehr, Verbesserung der Effektivität in Bezug auf die Zeit, Sicherung der Kundenzufriedenheit und der Sicherheit, Verbesserung der Effektivität in Bezug auf die Kosten, Schutz von Flugzeugen und Flugreisenden sowie Zukunftsforschung. Für das gesamte Programm TRANSPORT weist die PROVISO Auswertung 2014 (mit Datenstand 11/2013) aus, dass 2,4 \% der Projektanträge österreichischen Partnerorganisationen zugesprochen wurden. Per Stand 09/2010 waren es noch 1,2 \% im Ländervergleich der EU. Diese positive Entwicklung ist jedoch für den Luftfahrtsektor nicht repräsentativ. Wie die untenstehende Auswertung der FFG veranschaulicht, liegt der Luftfahrtsektor eindeutig darunter. In Luftfahrt AAT ist eine relativ niedrige Beteiligungs- und Rückflussquote, wenn auch mit steigender Tendenz, festzumachen. In Clean Sky 1 ist die Rückflussquote zwar zufriedenstellend, die Teilnahmen in Projekten ist allerdings, nach der Beobachtung durch die FFG, rückläufig.

Tabelle 3 Rückflussquoten aus FP7 und FP6 sowie European Clean Sky

\begin{tabular}{|l|r|r|r|c|}
\hline Programm & $\begin{array}{c}\text { Beantragt }(\boldsymbol{(}) \\
\text { gesamt }\end{array}$ & $\begin{array}{c}\text { Beantragt } \\
(\boldsymbol{(}) \text { durch AT }\end{array}$ & $\%$ & $\begin{array}{c}\text { Rückflussquote (\%) } \\
\text { naclh AT }\end{array}$ \\
\hline Luftfahrt AAT & 1.092 .336 .010 & 12.529 .865 & 1,15 & 57,4 \\
\hline Clean Sky & 200.953 .159 & 5.225 .473 & 2,60 & 130,0 \\
\hline Gesamt Luftfahrt FP7 & 1.293 .289 .169 & 17.755 .338 & 1,37 & 68,6 \\
\hline Gesamt Luftfahrt FP6 & 950.332 .839 & 10.522 .956 & 1,11 & 50,3 \\
\hline
\end{tabular}

Quelle: FFG; Luftfahrt AAT entspricht den Ausschreibungen der FP im Bereich Luftfahrt; Cleans Sky entspricht den Ausschreibungen, die über CS Joint Undertaking vergeben wurden (siehe unten). 
Horizon 2020, das neue Rahmenprogramm für Forschung und Innovation seit 2014, führt alle forschungs- und innovationsrelevanten Förderprogramme der Europäischen Kommission zusammen. Zurzeit sind Mittel in Höhe von $77 \mathrm{Mrd}$. Euro für das von 2014-2020 laufende Programm vorgesehen, davon rund 6,4 Mrd. Euro für den Bereich „Intelligenter, umweltfreundlicher und integrierter Verkehr“ (8,3 \%).

Innerhalb der Forschungsrahmenprogramme gibt es weitere, für die Luftfahrt bedeutsame Initiativen. So stellten im 7. Forschungsrahmenprogramm die so genannten Joint Technology Initiatives (JTI) ein neues, als Public-Private Partnership zwischen der EU-Kommission und der Industrie organisiertes Instrument dar. Eine von sieben Joint Technology Initiatives ist Clean Sky, die neue Technologien im Luftfahrzeugbau behandelt. Clean Sky (2008 - 2013) verfügte über ein Gesamtbudget von bis zu 1,6 Milliarden Euro (zur Hälfte Industrieanteil) und war damit weltweit das größte, einzelne Forschungsvorhaben in der Luftfahrtforschung.

SESAR (Single European Sky ATM Research Programme) ist eine von der Europäischen Kommission und EUROCONTROL im Jahr 2007 gestartete Initiative mit dem Ziel, bis 2020 ein EU-weit einheitliches Flugverkehrsmanagementsystem aufzubauen. Das Budget beläuft sich auf insgesamt 2,1 Milliarden Euro.

Mit GALILEO wird ein europäisches Satellitennavigationssystem aufgebaut, dass für zivile Zwecke genutzt werden soll. Das Projekt wird von der Europäischen Union und die Europäische Weltraumorganisation (ESA) durchgeführt.

Österreich ist auch Mitglied des ERA-Net AirTN, das dazu beiträgt, transnationale Ausschreibungen nationaler Förderprogramme der Mitgliedsstaaten zu organisieren. Durch Take Off werden nationale Anteile der ERA-Net AirTN Projekte gefördert.

\section{Österreichische Luftfahrtstrategie und -förderung}

Ausgehend von dem in Kapitel 2 skizzierten Rational wurde im Jahr 2002 das Förderprogramm Take Off, nach Durchführung einer ex-ante Evaluierung, gestartet. Im Zuge des Auslaufens der ersten Förderperiode wurde mit der FTI-Luftfahrtstrategie 2008 erstmals ein umfassender, strategischer Rahmen für die sich stark entwickelnde Zulieferindustrie des Luftfahrtsektors in Österreich dargelegt. Mit Adaptierungen wurde Take Off in die FTI-Luftfahrtstrategie 2008 des bmvit eingebettet und stellte daraufhin einen wesentlichen Pfeiler der Umsetzung dar. 
Die Programmperiode ab dem Jahr 2007 brachte folgende Neuerungen: eine Erhöhung der Ausschreibungsbudgets in den Jahren 2008/9 (danach ging das Volumen wieder deutlich zurück), eine Ausweitung der Zielgruppen, die Stärkung von Wertschöpfungsketten in Marktsegmenten, die Initiierung von Leitprojekten, Aufnahme der Instrumente Stimulierung (mittlerweile Sondierung) und kooperative Projekte der industriellen Forschung, die finanzielle Unterstützung von Ausbildungsprojekten für qualifizierte ForscherInnen und TechnikerInnen, sowie die Intensivierung kooperativer anspruchsvoller Forschungsprojekte mit ausländischen Partnern via ERA-Net AirTN.

\subsection{Programmziele und deren Kongruenz - Ein Logic Model}

Gemäß Selbstbeschreibung des bmvit bzw. der FFG wurden die ursprünglichen Schwerpunktsetzungen von Take Off nach der Erarbeitung der österreichischen Luftfahrtstrategie erweitert, und bilden seither folgendes Rational (siehe Programmdokument und diverse Jahresberichte, Kapitel 1): „Oberstes Anliegen ist dabei die Maximierung der Doppeldividende zur Erreichung gesellschaftspolitischer Bedürfnisse (wie Mobilitätsbedarf und Klimaschutz) einerseits, sowie die Setzung eines technologiepolitischen Schwerpunkts im Bereich Luftfahrttechnologien andererseits. Durch hohe Forschungs-, Technologie- und Kooperationskompetenz und durch Vernetzung und Know-how-Aufbau im industriellen, universitären und außeruniversitären Bereich soll die Wettbewerbsfähigkeit der österreichischen Luftverkehrswirtschaft und Luftfahrtzulieferindustrie weiter verbessert werden. Take Off unterstützt den Aufbau strategischer nationaler, europäischer und internationaler Partnerschaften sowie die Erschließung neuer Märkte. Damit sollen langfristig die Umsätze des österreichischen Luftfahrtsektors gesteigert und High-Tech-Produktionen in Österreich abgesichert werden, die wiederum sichere und hoch qualifizierte Arbeitsplätze generieren." Die folgenden vier übergeordneten Zielsetzungen wurden dabei mit Subzielen versehen:

1) Sicherung und Ausbau der Wettbewerbsfähigkeit der österreichischen Luftfahrtindustrie und -forschung durch:

- Förderung der Forschungs-, Technologie- und Kooperationskompetenz zwischen Wirtschaft und Wissenschaft

- Zeitgerechte Entwicklung und Weiterentwicklung neuer Technologien mit Luftfahrtrelevanz

- Auf- und Ausbau von Themenführerschaften (und damit Marktpositionierungsmöglichkeiten) in ausgewählten strategischen Technologiefeldern und thematische Fokussierung

- Entwicklung technisch und ökonomisch hervorragender Produkte und Dienstleistungen

- Steigerung des KMU-Anteils an den FuE-Aktivitäten in Take Off 
2) Unterstützung eines effizienten, sicheren, klimaschutz- und komfortorientierten Lufttransportsystems durch:

- Setzung entsprechender thematischer Forschungsschwerpunkte

- Berücksichtigung der Bedürfnisse der österreichischen Luftverkehrswirtschaft in FuE Themen

- Anknüpfung des Luftverkehrs an andere Verkehrsträger bzgl. Logistikfragen

3) Ausbildung qualifizierter ForscherInnen und TechnikerInnen und Intensivierung kooperativer anspruchsvoller Forschungsprojekte:

- Hohes Qualifizierungsniveau der Beschäftigten in Unternehmen

4) Verbesserung der europaweiten und internationalen Sichtbarkeit, sowie stärkere Vernetzung der österreichischen FuE-Community:

- Erfolgreiche Integration in/Teilnahme an den Leitprojekten SESAR und Clean Sky bzw. weiteren Demonstratorprojekten

- Erfolgreiche Integration der österreichischen Luftfahrtindustrie in die europäische Luftfahrtforschung (60\% Rückflussquote im 7. EU-Rahmenprogramm, Teilnahme am ERA-NET AirTN inklusive gemeinsamer Ausschreibungen

- Überleitung von Forschungsergebnissen in die Praxis

- Impulsmaßnahmen zum Aufbau von Kooperationsmöglichkeiten mit Amerika und Asien 


\section{Grafik $4 \quad$ Logic Model des Programms Take Off}

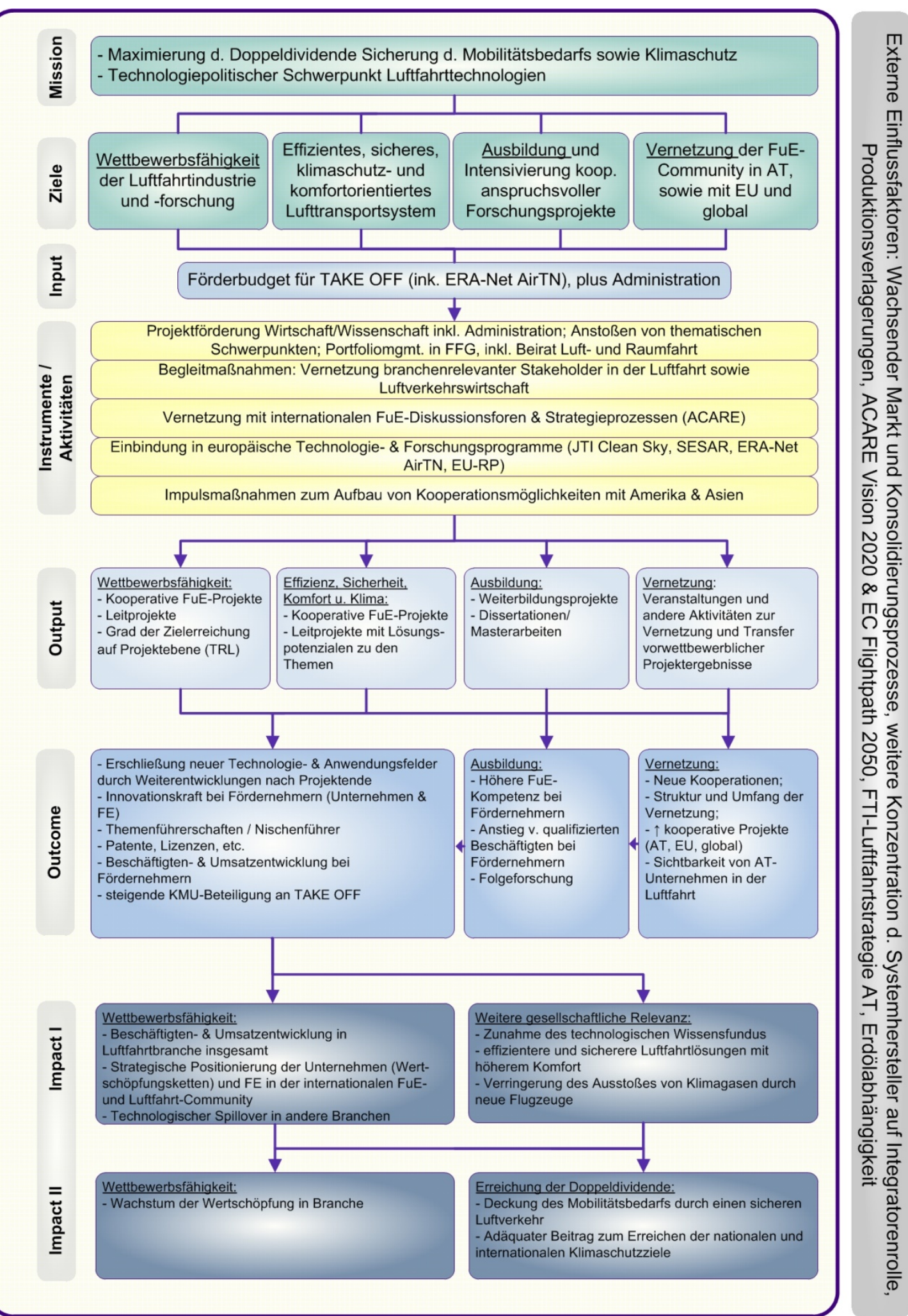

Quelle: KMU Forschung Austria und Partner, basierend auf Dokumenten und Interviews. 
Die Mission des Förderprogramms Take Off ist in die Luftfahrtstrategie der Bundesregierung eingebettet, die wiederum in die europäische Strategie eingebettet ist, und erscheint aufgrund dessen adäquat.

Die Ziele zur Operationalisierung der Mission decken die relevanten Systemelemente ab. Hier kann man anführen, dass diese Ziele noch expliziter formuliert werden könnten. Damit ist angesprochen, dass „effizient, sicher, klimaschutz- und komfortorientiert" zwar insgesamt sinnvolle Teilziele darstellen, jedoch nicht notwendigerweise zusammengehören und deshalb auch nicht zusammen diskutiert, und damit möglicherweise abgewertet werden sollten. Hier ist auf die separate Behandlung der Themen auf der Ebene der Outcomes und Impacts zu achten. Ob das Thema Komfort, das nur wenige Unternehmen in Österreich vordringlich anspricht, eine separate Ausweisung als Zielgröße rechtfertigt, sei ebenso zur Diskussion gestellt. Weiters könnten alle Ziele durch eine weitere Konkretisierung gewinnen, d.h. qualitativ oder quantitativ präzisiert werden. Zum Beispiel sind als übergeordnete Vorgaben für Effizienz, die Klimaschutz- sowie Sicherheitsziele die Festlegungen in VISION 2020 und Flightpath 2050 zu nennen.

Weiters ist die Zielsetzung der Ausbildung dahingehend kritisch zu hinterfragen, als mit Take Off in einigen Ausschreibungen die Weiterbildung von ForscherInnen und TechnikerInnen betrieben wurde. Als ein Vorgriff auf die später präsentierten Monitoringdaten lässt sich hier festhalten, dass die Finanzierung der Weiterbildung durchaus den Unternehmen selbst überlassen werden kann. Etablierte Wissenschaftlerlnnen und TechnikerInnen bilden sich anhand der konkreten, durchgeführten Projekte weiter und holen sich ihre weiteren spezifischen Defizite durch Rekrutierungen oder Kooperationen. Die projektbegleitende Ausbildung von Nachwuchskräften ist hingegen eine vielversprechende Strategie, da dies eine praxisorientierte Finalisierung eines Ausbildungsabschnittes sicherstellt, und erfahrungsgemäß relativ oft in eine direkte Rekrutierung in beteiligte Unternehmen resultiert. Dies scheint für die spezifischen technologischen Anforderungen der Luftfahrt(zuliefer)industrie besonders geeignet und könnte in Zukunft ausgeweitet werden. Hier sind Formate auf unterschiedlichen formalen Qualifikationsniveaus denkbar, und wären am besten nach Konsultation mit den Unternehmen festzulegen.

Zur Verfolgung der erwähnten Ziele wurden im Zeitraum 2002 - 2013 Inputs mit einem Fördervolumen von $€ 61,2$ Mio. bereitgestellt.

Die Aktivitäten und konkreten Instrumente zur Umsetzung des Programms umfassen alle typischen Maßnahmen eines Förderprogramms, plus der Schaffung eines Beirats zur strategischen Begleitung sowie starke Vernetzungsaktivitäten, die für einen (anfangs noch sehr) disparaten Sektor mit relativ starker KMU-Beteiligung als geeignet erachtet wurde. Hier sei noch das aktive Portfoliomanagement angesprochen, d. h. die zielgerichtete Beratung von Projektwerbern vor dem Hintergrund der Abgrenzung der Programme zueinander, das innerhalb des doch weitgefächerten FFG Förderportfolios nötig ist. 
Die Vernetzung der Unternehmen und Forschungseinheiten zur Potentialentwicklung und Transfer vorwettbewerblicher Projektergebnisse wurde als ein wichtiges Thema angesehen und neben den Kooperationsprojekten mittels Begleitmaßnahmen zum Take Off Programm anhand von verschiedenen Vernetzungsformen umgesetzt.

Die Vernetzung mit internationalen Foren und Programmen nimmt in einem derart langfrist-orientierten und strategischen Markt einen nicht unwesentlichen Teil des Programmmanagements dar, das de facto von bmvit und FFG zusammen verfolgt wird. Hierbei hat das Vorhandensein eines eigenen Luftfahrtprogramms die Teilnahme an ERA-Net erleichtert; so wurden z.B. gemeinsame Ausschreibungen mit insb. Deutschland durchgeführt, und daraufhin die nationalen Teile mit Take Off unterstützt.

Das Hauptaugenmerk lag bislang auf dem europäischen Markt; das in der Strategie und dem Programmdokument angesprochene Engagement in den USA sowie Asien wurde bislang eher den Unternehmen überlassen. Wie die Ö-LINKupdate Studie (Brimatech 2015) erhob, erreichten die Unternehmen knapp ein Drittel ihrer Umsätze in Nordamerika, $3 \%$ in Südamerika und weitere $7 \%$ in Asien. Speziell die BRIC Staaten und der asiatische Markt scheinen ausbaufähig, und wurde aufgrund dessen auch wieder in die Neufassung der FTI-Luftfahrt Strategie 2020+ aufgenommen.

Unmittelbare Programm-Outputs, Outcomes/Ergebnisse sowie Wirkungen

Als Outputs sind die unmittelbaren Ergebnisse der Aktivitäten und Maßnahmen für Kunden oder Stakeholder zu verstehen. Die Outcomes bzw. Ergebnisse sind mittelbare Effekte auf das Verhalten von Kunden oder Stakeholder (indem Sie etwas mit den Outputs tun und daraus einen Nutzen oder Schaden ziehen); diese sind auf der Mikroebene angesiedelt und haben tendenziell eine mittelfristige Komponente. Impacts bzw. Wirkungen weisen mittelbare, breitere Effekte auf einen größeren Adressatenkreis bzw. die Gesellschaft aus, und sind als logische Folge von Outputs über Outcomes bis zu Impacts hergeleitet; diese sind auf der Meso- bis Makroebene angesiedelt und haben eine mittel- bis langfristige Komponente. Man unterscheidet oft zwischen Wirkungsebene I (meso) und II (makro). Während die Outputs oft noch relativ einfach vom programmbegleitenden Monitoring erhoben werden, wird dies hinsichtlich Outcomes bis hin zu Impacts meist schwieriger bzw. aufwändiger. In der vorliegenden Evaluierung wurde die Aufbereitung dieses Themenbereichs mit einem Methodenmix aus der Analyse der Monitoringdaten, von Befragungen, Fallstudien zur Verfolgung von Wirkungsmechanismen, sowie einer Input-Output Analyse nachgegangen, und spiegelt sich in der Struktur des Berichts wider. Die damit einher gehenden qualitativen und quantitativen Indikatoren sind der Grafik 2 zu entnehmen.

\section{Externe Einflussfaktoren}

Die wichtigsten Determinanten der globalen Luftverkehrsnachfrage (Bräuninger et al. 2010, sowie FTI-Luftfahrtstrategie Österreich 2014) sind die weltwirtschaftliche ökonomische Entwicklung eingebettet in die Internationalisierung der Wirtschaftsströme 
sowie die zunehmende Urbanisierung, die starke Konkurrenz im Flugverkehr und fluktuierende Ölpreise, bei gleichzeitig steigenden Anforderungen an die Umweltwirkungen der Luftfahrt. Dies führt zu steigenden Auftragseingängen bei den großen Herstellern (OEM's) bei einer gleichzeitigen Konsolidierung der Zulieferketten. Die Herausforderung für die Zulieferindustrie besteht darin, Technologie- und Innovationsführerschaft in spezifischen Segmenten zu erreichen bzw. zu erhalten, um ihre Position in der Zulieferkette abzusichern.

Diese Rahmenbedingungen deuten auf eine potenzielle, mittelfristige Volatilität in einem grundsätzlich stark wachsenden Markt hin, die jedoch in den diversen Luftfahrtstrategien gut abgebildet sind. Speziell die Anforderungen an die Reduzierung von Umweltwirkungen der Luftfahrt impliziert eine Forschungsstrategie mit möglicherweise hohen Unsicherheiten und Risiken. Die vornehmlich als KMU strukturierte Unternehmerschaft in Österreich sieht sich in diesem langfristig orientierten Markt unterschiedlichen Herausforderungen gegenüber, die durch die Unterstützung durch die öffentliche Hand teilweise abgefedert werden kann. Hier sind sowohl Argumente des Marktversagens (Reduzierung von Unsicherheit/Risiko, Ausgleich von Informationsdefiziten, Unteilbarkeit von Forschungsprojekten als Herausforderung für insb. $\mathrm{KMU}$, etc.) also auch des Systemversagens (Interaktion zwischen Wissenschaft und Unternehmen, wissenschaftliche Infrastruktur, Kompetenzen, etc.) als eine Begründung für die Intervention der öffentlichen Hand heranzuziehen.

\subsection{Einbettung in das restliche FFG Portfolio}

Gemäß der Empirie ist das Programm mit dem größten Naheverhältnis das Basisprogramm. Im Zeitraum 2002 bis 2013 stehen 142 Projekte mit einem Förderbarwert von $€ 61,2$ Mio. in Take Off weiteren 116 luftfahrtrelevanten Projekten (Zeitraum 1999-2013) mit einem Förderbarwert von € 41,4 Mio. im Basisprogramm gegenüber. Ein Unterschied liegt darin, dass im Basisprogramm vorwiegend Einzelprojekte (mit einem höheren Eigenanteil) unterstützt wurden, während Take Off einen klaren Schwerpunkt auf kooperative FuE Projekte legt.

In anderen Programmen der FFG wurden weitere 29 Projekte mit einem Förderbarwert von $€ 18,5$ Mio. für die Luftverkehrswirtschaft unterstützt. Ein fallweise beanspruchtes Programm ist hier z.B. die „Produktion der Zukunft", wobei hier die Schwerpunktsetzung gemäß der Programmträger bei Prozessen (PdZ) bzW. Produkt-Teilen (Take Off) liegen sollte. Aufgrund der Fallstudien und Interviews konnten wir feststellen, dass dies bei den Unternehmen nicht so einfach zu trennen ist, da oft ohne einen neuen Teil auch kein neuer Prozess entwickelt wird. Dadurch herrscht bei manchen Unternehmen der Eindruck vor, dass man beide Programme mit ähnlichen Themen adressieren kann; der hauptsächliche Unterschied liegt dann in den unterschiedlichen Erfolgswahrscheinlichkeiten, den Förderbarwerten sowie der spezifischen Unterstützung durch die ProgrammmanagerInnen. 
Manche Unternehmen der Luftfahrtbranche sind auch in Kompetenzzentren involviert. Hier kann es z.B. durchaus vorkommen, dass im Rahmen von Take Off vorangetriebene Entwicklungen dann im Rahmen des Kompetenzzentrums weitergetragen werden. In einem konkreten Fall konnten wir nachvollziehen, dass die Forschungsergebnisse aus Take Off in einem Kompetenzzentrum von zwei der ursprünglich beteiligten Unternehmen zu einem fertigen Produkt weiterentwickelt wurde und nun vertrieben wird.

Wir können damit festhalten, dass die Intention des Programmeigners, nämlich die spezifische Unterstützung der noch jungen „Branche“ Luftfahrtzulieferindustrie mit einem eigenständigen Programm durchaus ein aktives Portfoliomanagement benötigt, d. h. die zielgerichtete Beratung von Projektwerbern vor dem Hintergrund der Abgrenzung der Programme zueinander, da Interaktionen und Überlappungsbereiche mit anderen Programmen gegeben sind. 


\section{Von Inputs zu Outputs: Aktivitäten und Leis- tungen des Programms}

\subsection{Förderinanspruchnahme}

Zur Verfolgung der erwähnten Ziele wurden folgende Inputs im Zeitraum 2002 2013 bereitgestellt: Im Rahmen von 11 Ausschreibungen innerhalb von Take Off wurden 142 Projekte mit einem Fördervolumen von $€$ 61,2 Mio. gefördert. Das Gros der Fördermittel wurde dabei in den Jahren nach der Erstellung der FTILuftfahrtstrategie zur Verfügung gestellt. Nahezu drei Viertel der Förderbarwerte sind in der zweiten Hälfte des Beobachtungszeitraumes in Anspruch genommen worden, wenngleich sich das jährliche Ausmaß variabel darstellte. Mit rd. € 9 Mio. wurde die höchste Fördersumme im Jahr 2008 aufgrund der damaligen Luftfahrtstrategie bereitgestellt (2009: 7,04 Mio., 2010: 5 Mio., 2011: keine Ausschreibung, 2012: 6 Mio., 2013: 4 Mio., 2014: 8 Mio. (Verdoppelung auf Basis der neuen Luftfahrtstrategie).

Im Rahmen der Take Off Evaluierung wurden seitens der FFG Projekt- und Unternehmensdaten zur Verfügung gestellt, welche die folgende Beschreibung der Datenstruktur ermöglichen. Dabei wurde der Beobachtungszeitraum auf das Jahr 2014 ausgeweitet. Dementsprechend werden nachfolgend sämtliche Projekte betrachtet, die zu Beginn der Evaluierung (per Juni 2014) genehmigt waren.

Im Fokus stehen 411 Projektteilnahmen, die den Ausgangspunkt für die weitere Analyse bilden. Die Förderfälle lassen sich auch bezüglich der durchführenden Organisationstypen unterscheiden: davon sind 262 Projektbeteiligungen dem Unternehmensbereich zuzuordnen und 149 den Forschungseinrichtungen (FE: universitäre oder außeruniversitäre Forschungseinrichtungen, Kompetenzzentren, FHs, Fachverbände). Dass die geförderten Projekte überwiegend in Form von Forschungskooperationen durchgeführt wurden (Kooperationserfordernis ab der 4. Ausschreibung) zeigt sich bei Berücksichtigung der Fördernehmerzahl. Die 411 Förderfälle teilen sich auf 187 unterschiedliche Fördernehmer (132 Unternehmen und 55 Forschungseinrichtungen) und 151 Projekte auf. In 136 der 151 Projekte hat daher zumindest ein Unternehmen und in 94 Projekten hat zumindest eine Forschungseinrichtung teilgenommen. Die 15 Projekte ohne Unternehmensbeteiligung teilen sich auf Humanressourcenentwicklung, Studien, sowie Projekte durch Intermediäre auf. 


\section{Grafik 5 Take Off Programmbeteiligungen}

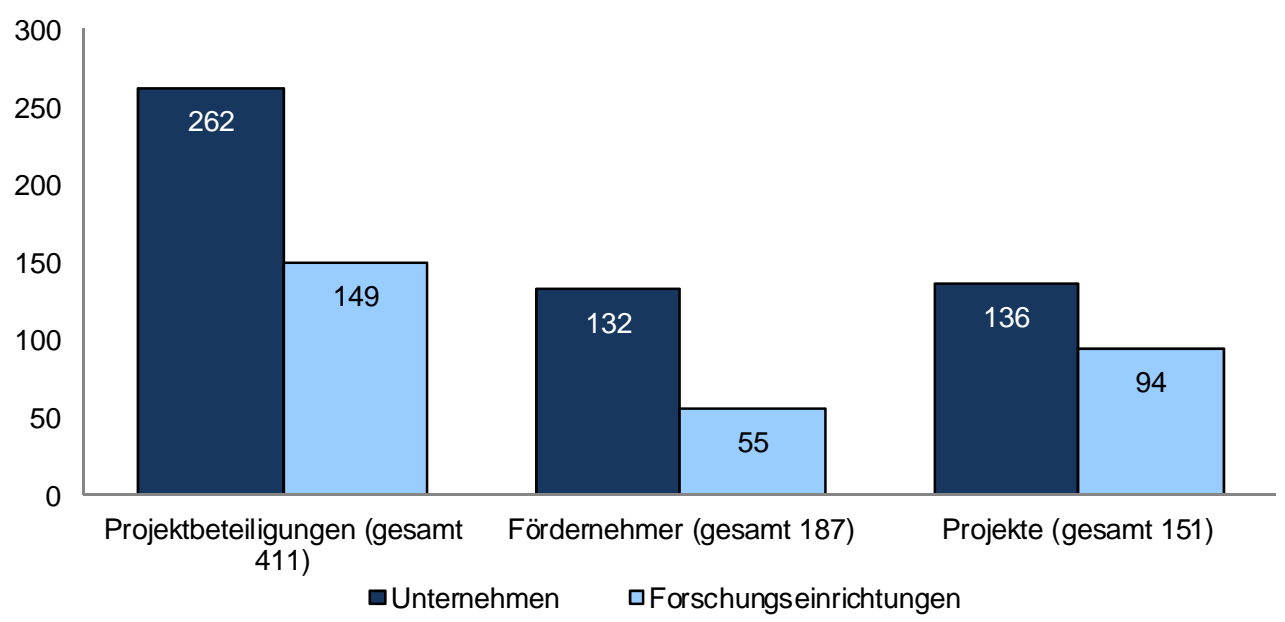

Quelle: FFG Projektdatenbank, $\mathrm{n}=411$ Projektbeteiligungen im Rahmen von 151 Projekten (ohne Begleitmaßnahmen, aber mit Aufwendungen)

Die Analyse der FFG Monitoringdaten ermöglicht des Weiteren die Zeichnung eines detaillierten Bildes von der Verwendung der bereitgestellten, öffentlichen Budgetmittel. Seit Inkrafttreten des Take Off Förderprogramms wurden im Rahmen von 11 Ausschreibungen 151 Forschungsprojekte plus (45) Begleitmaßnahmen durchgeführt. Diese umspannen den Zeitraum von 2002 bis 2013 und 11 thematische Anwendungsfelder (inkl. Humanressourcen, Querschnittsthemen und Begleitmaßnahmen). In der untenstehenden Grafik ist die Verteilung der eingesetzten Fördermittel auf diese Anwendungsfelder dargestellt. Darin nicht enthalten sind die Begleitmaßnahmen, die auf Grund der geringen Fördersummen sowie ihres lediglich unterstützenden Charakters ${ }^{2}$ nicht in die weitere Untersuchung eingeflossen sind. Die verbliebenen 151 Projekte bzw. die 411 damit verbundenen Projektbeteiligungen umfassen Gesamtförderungen in der Höhe von $€$ 65,1 Mio. Der größte Teil davon ist dem Themenbereich „komplexe Flugzeugstrukturen und Bauteile“ zuzurechnen (rd. $26 \%$ ), der sich aus 25 Forschungsprojekten bzw. 93 Projektteilnehmern zusammensetzt. Daraus ergibt sich eine durchschnittliche Fördersumme von rd. € 667.000 pro Projekt bzw. rd. € 179.000 pro Projektteilnehmer. Ebenfalls ressourcenintensiv (rd. $18 \%$ ) ist das Forschungsgebiet „Triebwerke“, in dem 52 Fördernehmer in 21 Projekten organisiert waren ( $r$ d. $€ 547.000$ pro Projekt bzw. rd. € 221.000 je Fördernehmer). Im Verhältnis zu der Zahl der durchgeführten Forschungsprojekte in den jeweiligen Themenbereichen ergibt sich die durchschnittlich höchste Fördersumme

2 Darunter fallen etwa Unterstützungsleistungen für die Durchführung von Vernetzungsveranstaltungen oder Präsentationen. 
im Bereich der intelligenten Fluggeräteinfrastruktur. Die durchschnittliche Förderung der acht Forschungsprojekte dieses Schwerpunktes beläuft sich auf rd. €766.000. Die geringsten Fördersummen pro Projekt bzw. Fördernehmer lassen sich im Bereich der Humanressourcen und Querschnittsthemen feststellen die jedoch rd. $29 \%$ der Projekte ausmachen. 44 Projekte wurden in diesen Bereichen abgewickelt, wobei diese ergänzend zu den technischen Fragestellungen der Take Off Forschungsprojekte zu verstehen sind. Zusätzlich zu den Förderungen im Rahmen von Take Off sind Unternehmen und FE mit Forschungsprojekten aus dem Bereich der Luftfahrt auch im Zuge der Basisprogramme gefördert worden. Auf Grund der unterschiedlichen Förderlogik der Programme greift letzteres vor allem FuE-Vorhaben auf, die bereits ein höheres TRL aufweisen bzw. Verwertungspotenziale bereits erkennbar sind.

Grafik 6 Anteil der Fördersumme bzw. Projektzahl nach Marktsegmenten

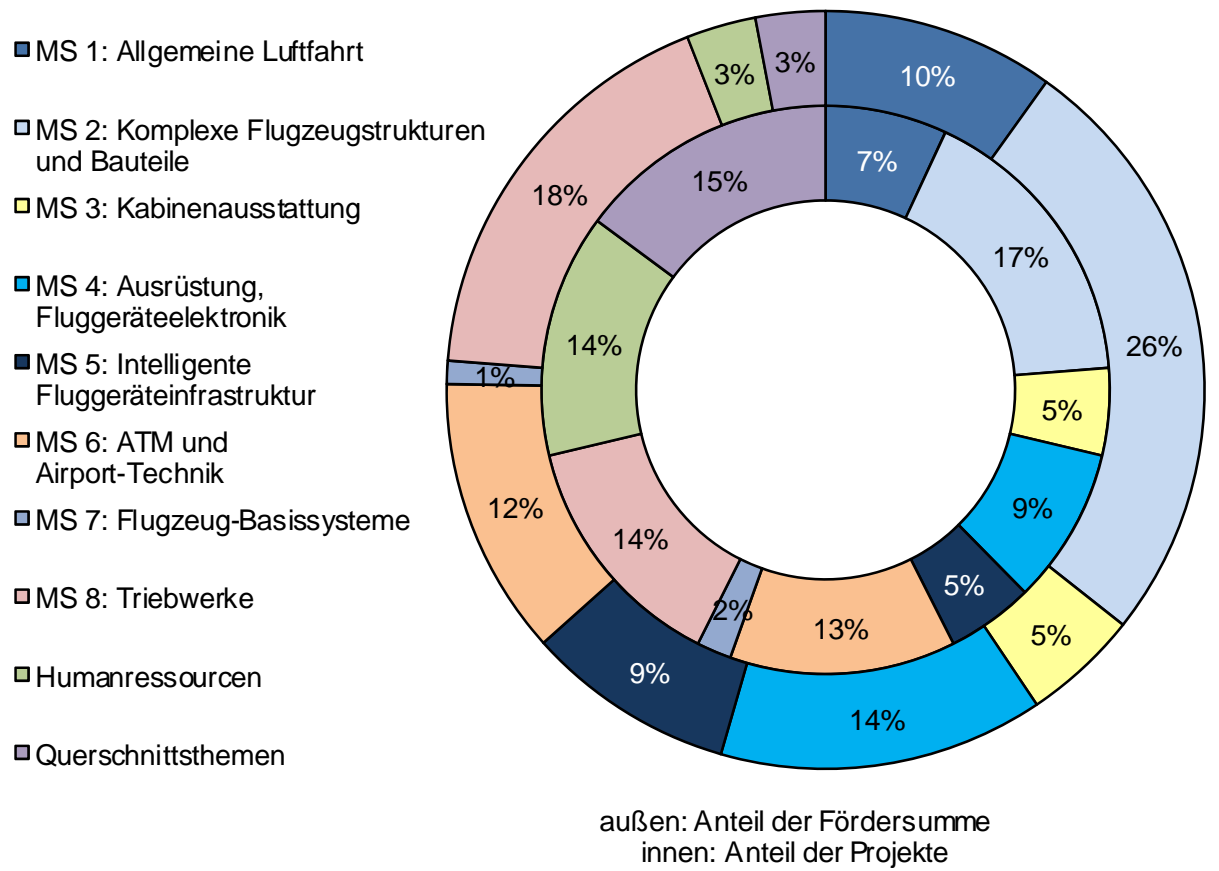

Quelle: FFG Projektdatenbank, n=411 Projektbeteiligungen im Rahmen von 151 Projekten (ohne Begleitmaßnahmen) 
Knapp zwei Drittel aller Projektbeteiligungen wurden von Unternehmen wahrgenommen. Noch etwas höher (rd. 68 \%) liegt der Anteil der Fördermittel, den diese erhalten haben. Außerhalb des Unternehmensbereiches sind rd. ein Drittel der Fördernehmer angesiedelt, wobei sich die Projektteilnahmen zum größten Teil auf Hochschulen (19\%) und Forschungseinrichtungen (rd. $16 \%)$ konzentriert. Ein geringer Anteil $(<1 \%)$ der Projekte wurde darüber hinaus unter Beteiligung von Fachverbänden oder Sonstigen durchgeführt. Die etwas höhere Beteiligung von Hochschulen an den untersuchten Forschungsprojekten drückt sich jedoch nicht gleichermaßen in einer höheren Fördersumme aus. Der Anteil der ausbezahlten Zuschüsse (rd. 15,5\%) liegt leicht unter jenem der Forschungseinrichtungen (15,7 \%).

Grafik 7 Take Off Projektbeteiligungen nach Anzahl der Projektträger sowie nach Summe des Barwerts, 2002-13

Anzahl der Projektteilnehmer

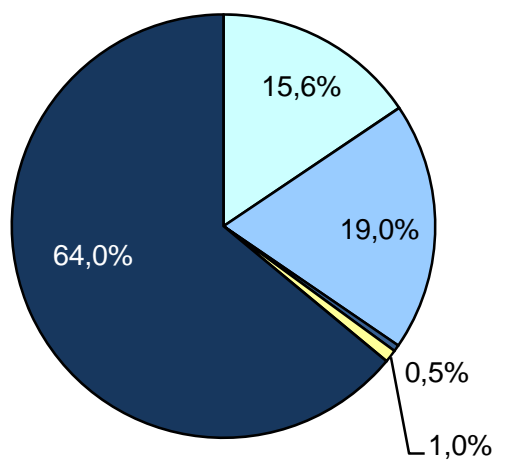

Summe der Barwerte

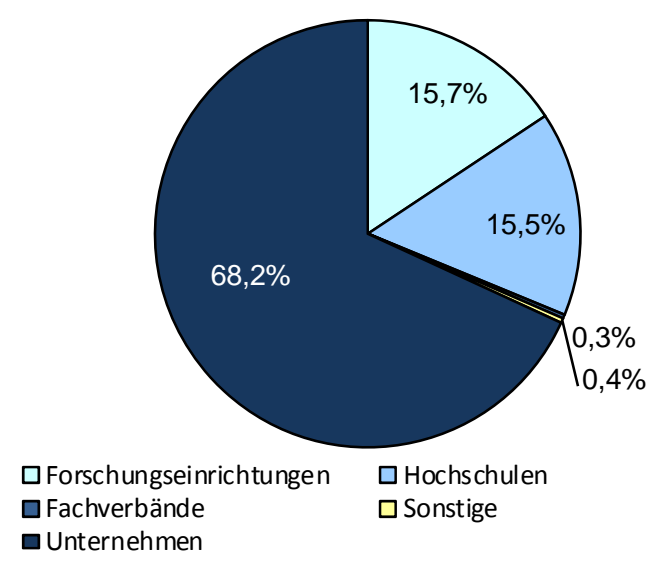

Quelle: FFG Projektdatenbank, $\mathrm{n}=411$ Projektbeteiligungen 
Darüber hinaus können die geförderten Unternehmen bezüglich ihrer Größe differenziert werden. Kleinunternehmen konnten rd. $20 \%$ der Projektteilnahmen auf sich vereinen und erhielten dabei rd. $12 \%$ des Fördervolumens. Dieses Verhältnis kehrt sich bei größeren Unternehmen um. Das gilt insbesondere für die Großunternehmen, die für knapp $27 \%$ aller Projektbeteiligungen rd. $38 \%$ der Fördermittel eingeworben haben. Unternehmen von mittlere Größe stellen einen Anteil von etwa $13 \%$ der Fördernehmer und haben zur Erfüllung der angestrebten Forschungsziele rd. $15 \%$ der bereitgestellten Fördersumme erhalten.

\section{Grafik 8 Fördernehmer nach Größenklassen und Summe Barwert der Förderung,} 2002-13

Anzahl der Projektteilnehmer

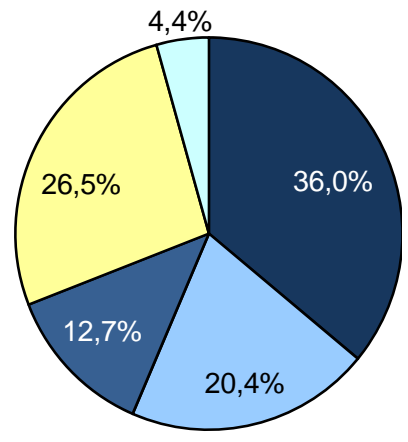

Summe der Barwerte

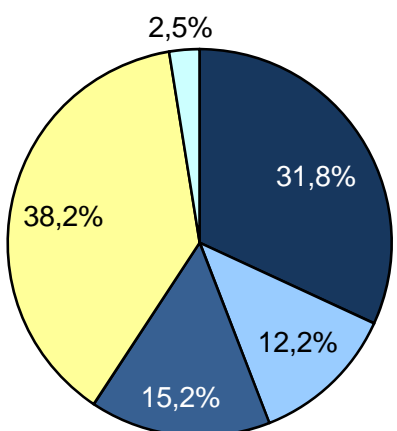

口FE, Hochschulen, Fachverbände, etc. 口Kleinunternehmen

Mittlere Unternehmen

口Großunternehmen

口kein KMU Status zuweisbar

Quelle: FFG Projektdatenbank, n=411 Projektbeteiligungen; KMU-Status nach EU Definition 
Betrachtet man die Projektteilnahmen der unterschiedlichen Organisationstypen im Zeitverlauf, so ist vorab zu erwähnen, dass es in den ersten vier Ausschreibungen noch kein Kooperationserfordernis im Rahmen von Projektanträgen gab. Die folgende Grafik veranschaulicht eine Verschiebung der Fördernehmerstruktur über die Jahre. Lag der Schwerpunkt der ersten drei Ausschreibungen aus Sicht der Unternehmen noch im Bereich der Großunternehmen (Anteil > $40 \%$ ), so rückten die KMU mit Beginn der 4. Ausschreibung verstärkt in den Mittelpunkt der Fördertätigkeiten. In den letzten beiden Ausschreibungsjahren 2012 und 2013 betrug der Anteil der GU nur mehr rd. ein Fünftel bzw. wurden etwa ein doppelt so hoher Anteil an KMU gefördert. Auch der Anteil der teilnehmenden Forschungseinrichtungen variierte im Zeitverlauf, wobei die Schwankungsbreite rd. $10 \%$ Prozentpunkte beträgt und um $32 \%$ bis $42 \%$ liegt. Ein deutlich niedrigerer Wert wurde im Zuge der ersten Ausschreibung erzielt.

Grafik 9 Anteil der Projekteilnahmen nach Forschungseinrichtungen, KMU, und Großunternehmen, Ausschreibungen 2002-13

10. Ausschreibung 2013

9. Ausschreibung 2012

8. Ausschreibung 2010

7. Ausschreibung 2009

6. Ausschreibung 2008

5. Ausschreibung 2008

4. Ausschreibung 2007

Offensivmittel 05/06

3. Ausschreibung 2004

2. Ausschreibung 2003

1. Ausschreibung 2002

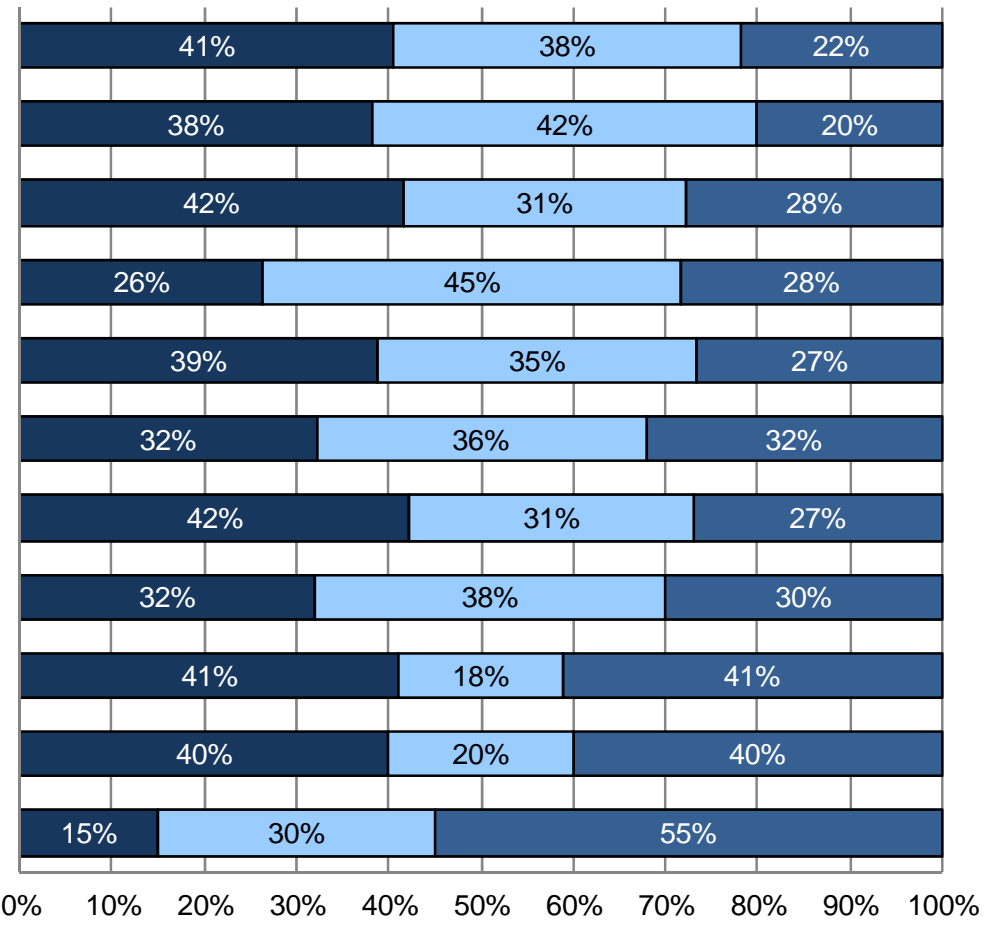

口FE, Hochschulen, Fachverbände, etc. पKMU घGroßunternehmen 
Noch deutlicher zeigt sich die stärker werdende Unterstützung der KMU und FE, zieht man die Verteilung der ausbezahlten Fördersumme heran. Die Dominanz der Großunternehmen in den ersten drei Ausschreibungsjahren reduzierte sich in weiterer Folge kontinuierlich und sank schließlich auf rd. ein Fünftel bis ein Viertel der zur Verfügung gestellten Gelder. Darüber hinaus pendelt sich die Auszahlung der eingesetzten Fördermittel zwischen Unternehmen und FE in den letzten drei Ausschreibungen etwa zu gleichen Teilen ein. Letztlich konnten die Forschungseinrichtungen sogar den größeren Anteil des Förderbudgets für sich lukrieren.

\section{Grafik 10 Förderbudgets nach Ausschreibung und Organisationstyp der Förder- empfänger}

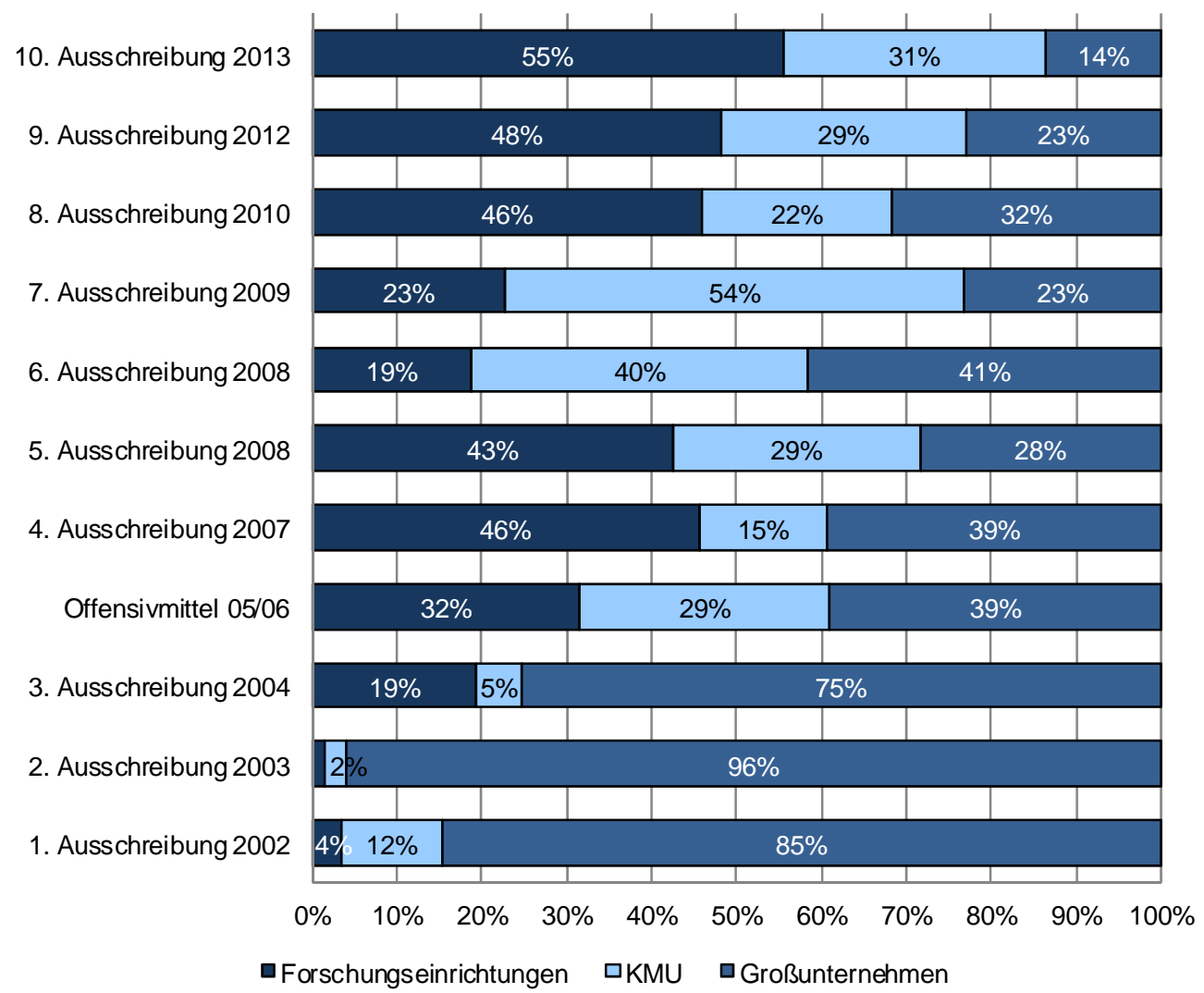

Quelle: FFG Projektdatenbank, n=411 Projektbeteiligungen; KMU-Status nach EU Definition 
Die regionale Verteilung der Projektbeteiligungen zeigt eine Konzentration der Fördernehmer in Oberösterreich, Steiermark, Wien und Niederösterreich. In diesen vier Bundesländern wurden $86 \%$ sämtlicher Take Off Beteiligungen abgewickelt, wobei rd. jedes vierte Projekt mit Beteiligung aus Oberösterreich durchgeführt wird. Regionale Zentren der österreichischen Luftfahrtindustrie lassen sich dementsprechend in Wien, Graz und Linz erkennen. Die Beteiligungen von Fördernehmern aus Tirol, Salzburg, Kärnten und dem Burgenland liegt im niedrigen einstelligen Bereich. Zusätzlich zu der Beteiligung österreichischer Unternehmen und Forschungseinrichtungen wurden fallweise auch deutsche und italienische Projektpartner hinzugezogen.

Grafik 11 Regionale Verteilung nach Projektbeteiligungen, 2002-13

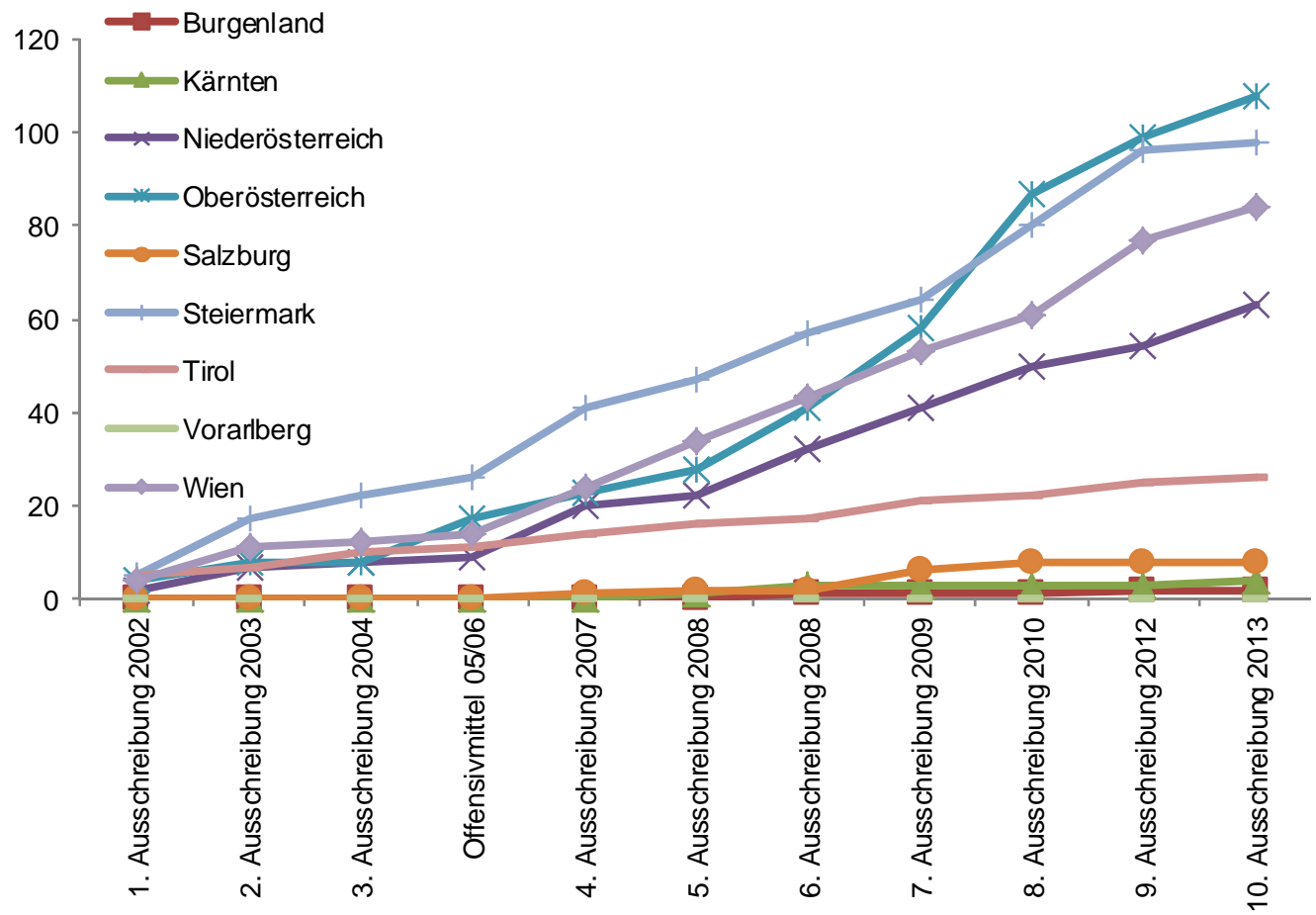

Quelle: FFG Projektdatenbank, $\mathrm{n}=411$ Projektbeteiligungen

Bei Betrachtung des Gründungsjahres der Unternehmen zeigt sich, dass die Branche der Luftfahrt von jüngeren Unternehmen geprägt ist. Mehr als 40 \% (76 Fördernehmer) der beteiligten Unternehmen und Forschungseinrichtungen wurden erst nach der Jahrtausendwende gegründet. Umgekehrt sind lediglich $13 \%$ (24) der geförderten Organisationen vor 1980 entstanden. Einem Fünftel der 187 beteiligten Unternehmen und Forschungseinrichtung konnte hingegen kein Gründungsjahr zugeordnet werden. 
Grafik 12 Anzahl von Teilnehmern und Anteil von Erstteilnehmern an Take Off

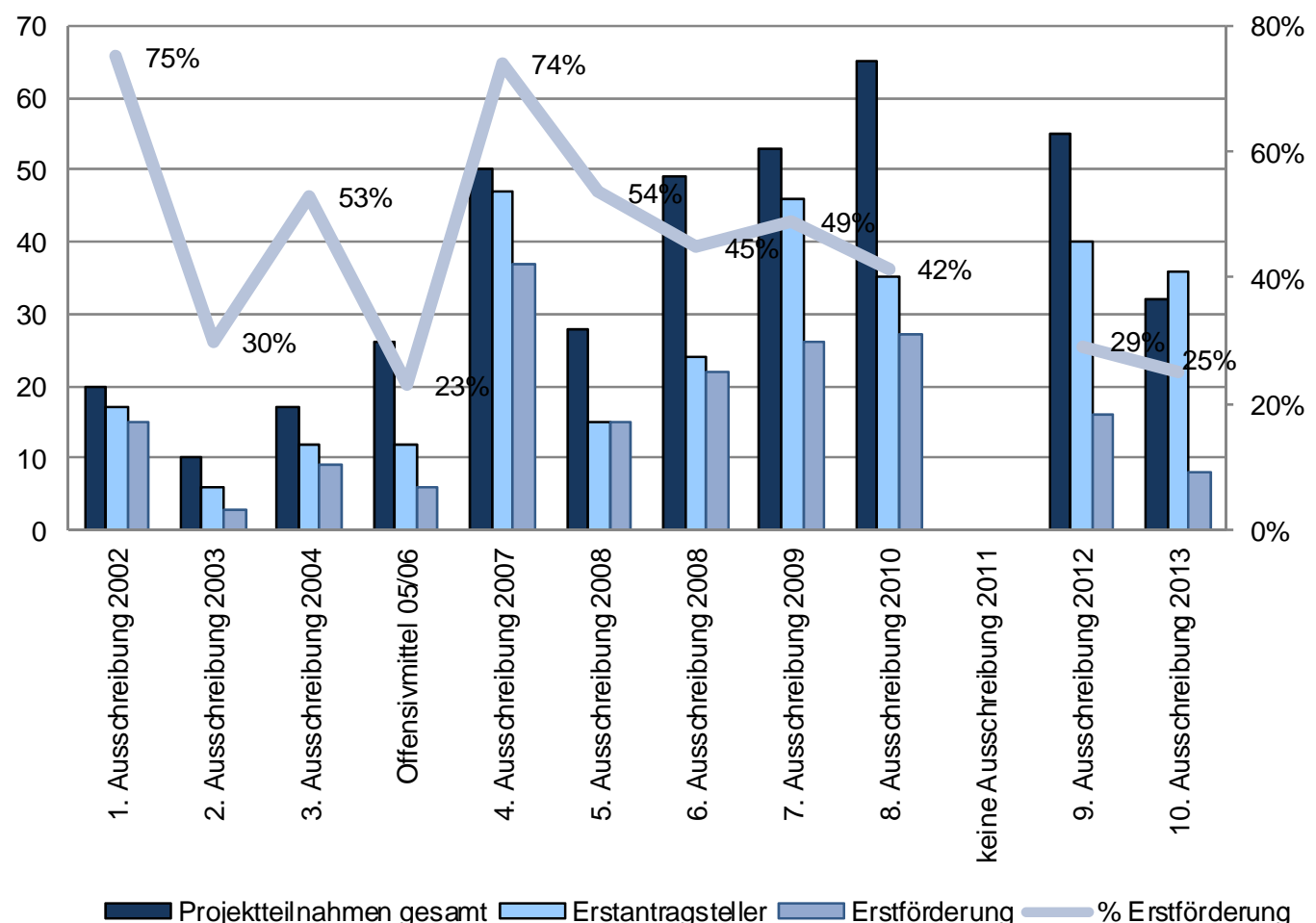

Quelle: FFG Projektdatenbank, n=411 Projektteilnahmen

In der obenstehenden Grafik ist die Zahl der geförderten Unternehmen und Forschungseinrichtungen gesamt, die Zahl der Erstantragsteller sowie der Erstgeförderten abgebildet. Unschwer lässt sich erkennen, dass im Zeitverlauf die Zahl der Projektteilnehmer tendenziell angestiegen ist, wobei der Höhepunkt in Folge der beiden Ausschreibungen im Jahr 2008 erreicht werden konnte. Auch in den Jahren 2009/10 konnten noch Projekte mit jeweils 50 bis 65 Projektteilnehmern gestartet werden. In weiterer Folge nahm die Zahl der geförderten Unternehmen und Forschungseinrichtungen aufgrund der wieder abnehmenden Budgets ab, und es ging eine Schere zwischen Erstantragstellern und Erstgeförderten auf. Der Grund liegt darin, dass sich immer mehr "neue“ Unternehmen (und FE) um die knapper werdenden TAKE OFF Mittel bewarben. Schließlich wurden im Zuge der letzten Ausschreibung 32 Fördernehmer unterstützt.

Der Anteil an erfolgreichen Erstantragsstellern schwankte anfangs stark. Dass die Zahl der Erstgeförderten im Jahr 2002 nicht $100 \%$ beträgt, ist auf die mehrfache Teilnahme einzelner Unternehmen und Forschungseinrichtungen zurückzuführen. Die relativ hohe Anzahl an Erstantragstellern der letzten Jahre legt nahe, dass es dem TAKE OFF Management gelang, zusätzliche Unternehmen anzusprechen. 
Grafik 13 Kumulierte Zahl der Projektbeteiligungen nach Marktsegmenten

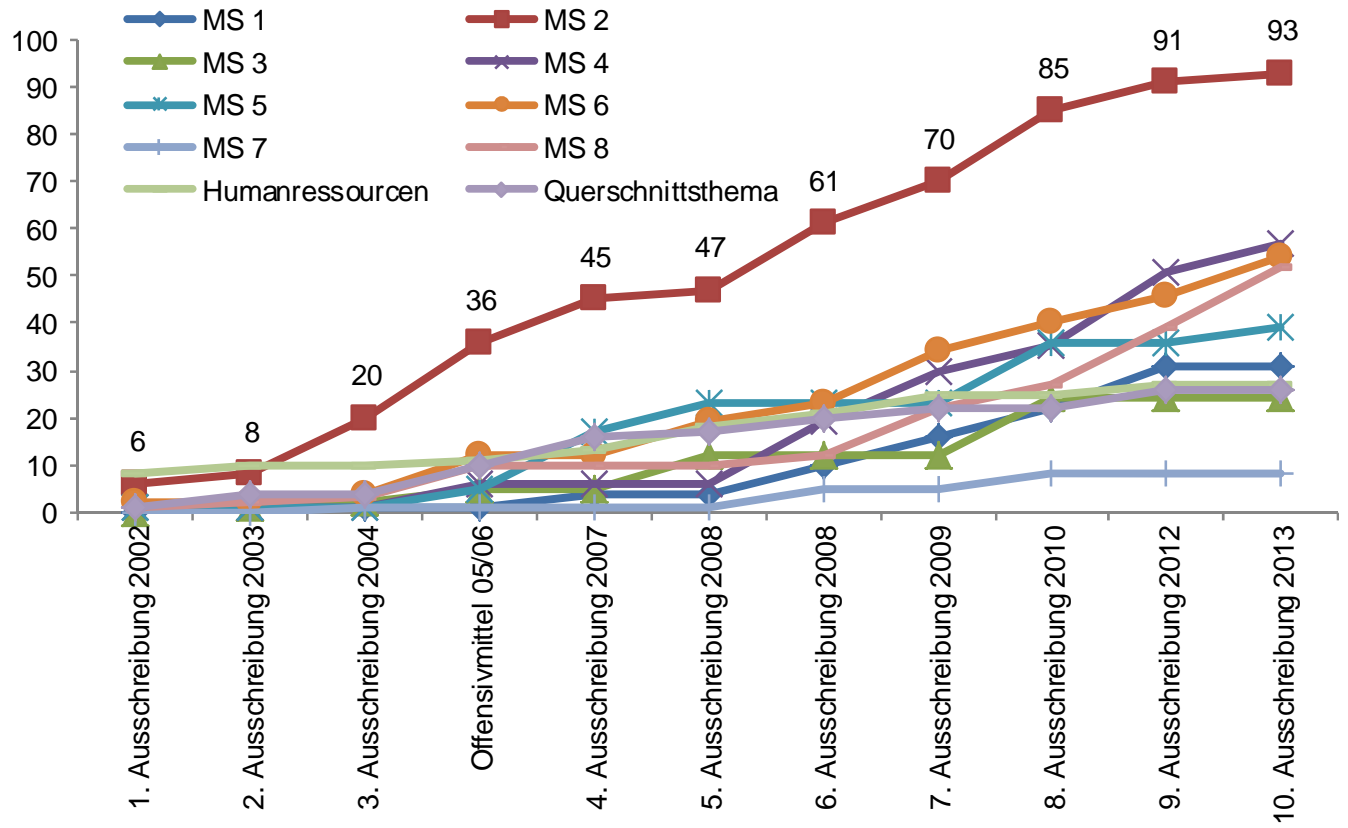

Quelle: FFG Projektdatenbank, n=411 Projektbeteiligungen

Die Zahl der Projektbeteiligungen hat seit dem Ausschreibungsjahr 2008 deutlich zugenommen und ist in obiger Grafik über die Jahre aggregiert dargestellt. Die häufigsten Beteiligungen sind im Marksegment der komplexen Flugzeugstrukturen und Bauteile (MS 2) erkennbar, in dem nahezu ein Viertel aller Projektbeteiligungen konzentriert ist. Aber auch in den Marktsegmenten Ausrüstung und Fluggeräteelektronik (MS 4), ATM und Airport-Technik (MS 6) sowie Triebwerke (MS 8) stieg das Interesse an Take Off Förderungen seit 2008 deutlicher an als in den verbliebenen Themenbereichen. Die Weiterbildung von Unternehmensmitarbeitern sowie Forschern als ein separates, förderwürdiges Thema wurde initial definiert, wurde jedoch nur bis 2009 ausgeschrieben. Dementsprechend bleibt die Zahl der HumanressourcenProjekte nach 2010 unverändert. 
Tabelle 4 Weitere Luftfahrt-Beteiligungen im FFG Portfolio nach Organisationstyp (in genehmigten Projekten 1999-2013)

\begin{tabular}{|l|r|r|r|r|r|r|}
\hline Bereich & $\begin{array}{c}\text { Unter- } \\
\text { nehmen }\end{array}$ & $\begin{array}{c}\text { Hoch- } \\
\text { schulen }\end{array}$ & $\begin{array}{c}\text { Forschungs- } \\
\text { einrichtungen }\end{array}$ & $\begin{array}{c}\text { Intermedi- } \\
\text { äre }\end{array}$ & Sonstige & Gesamt \\
\hline BP & 129 & 8 & 6 & & 1 & 144 \\
\hline SP & 55 & 10 & 12 & & 1 & 78 \\
\hline $\begin{array}{l}\text { Sonstige } \\
\text { TP }\end{array}$ & 29 & 10 & 13 & 1 & 3 & 56 \\
\hline Gesamt & $\mathbf{2 1 3}$ & $\mathbf{2 8}$ & $\mathbf{3 1}$ & $\mathbf{1}$ & $\mathbf{5}$ & $\mathbf{2 7 8}$ \\
\hline
\end{tabular}

Quelle: FFG Projektdatenbank

In der obenstehenden Tabelle sind die Beteiligungen der im Zuge von Take Off geförderten Unternehmen und FE an weiteren Förderprogrammen zusammengefasst. Insgesamt haben 213 Unternehmen und 65 Forschungseinrichtungen in weiteren Programmlinien der FFG partizipiert und zusätzlich Fördermittel in Höhe von rd. € 59,9 Mio. zur Bewältigung des Forschungsaufwandes lukriert. Die 144 Beteiligungen (in 116 Projekten) im Basisprogramm wurden in 129 Fällen von Unternehmen bzw. 15-mal von Forschungseinrichtungen durchgeführt. Im Rahmen der Strukturprogramme können den Take Off Fördernehmern weitere 78 Projektbeteiligungen zugerechnet werden. Dabei lässt sich eine anteilsmäßig höhere Beteiligung der Forschungseinrichtungen gegenüber dem Basisprogramm feststellen, der ebenso im Bereich der Thematischen Programme erkennbar ist. 


\subsection{Sicht der Unternehmen und Forschungseinrichtungen} Input-Additionalität

Die Durchführung der Förderprojekte hat dazu geführt, dass über die Gesamtsumme der Projekte (Förderung plus Eigenanteil) hinaus Mittel von den Unternehmen und Forschungseinrichtungen z.B. für Anschaffungen von Geräten oder Lizenzen, Einstellung von zusätzlichem Personal, etc. bereitgestellt wurden. Die befragten Forschungseinrichtungen berichten von der Bereitstellung von zusätzlich insgesamt 301.000 Euro und die Unternehmen von insgesamt 2.899.000 Euro. Dies entspricht $20 \%$ der ausgezahlten Fördersumme der Antwortenden, die zusätzlich investiert wurden. Die Differenzen der zusätzlichen aufgebrachten Mittel zwischen Forschungseinrichtungen und Unternehmen können als Hinweis auf die unterschiedlichen Verwertungsfähigkeit bzw. -aktivität der Fördernehmer interpretiert werden. Rund $85 \%$ der zusätzlichen Investitionen sind in Projekte geflossen, die bereits wirtschaftlich verwertet werden und ökonomische Effekte hervorgebracht haben bzw. von denen dies in den kommenden Jahren erwartet wird.

\section{Zielerreichung der Projekte}

Das wichtigste Projektziel für die von Unternehmen durchgeführten Forschungsvorhaben war die Entwicklung neuer Technologien. Mehr als die Hälfte der befragten Teilnehmer gab an, dass dieses Projektziel sehr wichtig für die Unternehmen war. Jedoch gibt nur rd. ein Viertel der Antwortenden an, dass es in ebenso hohem Maße erreicht werden konnte, wie ursprünglich angestrebt. Insgesamt wurde dieses Ziel jedoch in hohem Maße erreicht: über $60 \%$ geben eine hohen Zielerreichung an (Werte 1 und 2 zusammen betrachtet). Diese Ergebnisse verdeutlichen, dass die Entwicklung neuer Technologien mit Risiken behaftet ist und nicht jedes Projekt in vollem Umfang so wie geplant durchgeführt werden kann. Weniger stark erreicht als ursprünglich angestrebt wurden auch die eher betriebswirtschaftlich orientierten Ziele, die eigene Marktposition zu verbessern und neue Märkte zu erschließen. Die Aktivitäten innerhalb von FuE-Projekten sind hierzu weniger geeignet als zum Beispiel zum Aufbau neuer FuE-Partnerschaften, einem Ziel, das im Verhältnis zum ursprünglichen Plan sogar übererfüllt wurde. 
Grafik 14 Welche Projektziele wurden verfolgt bzw. erreicht (Unternehmen)

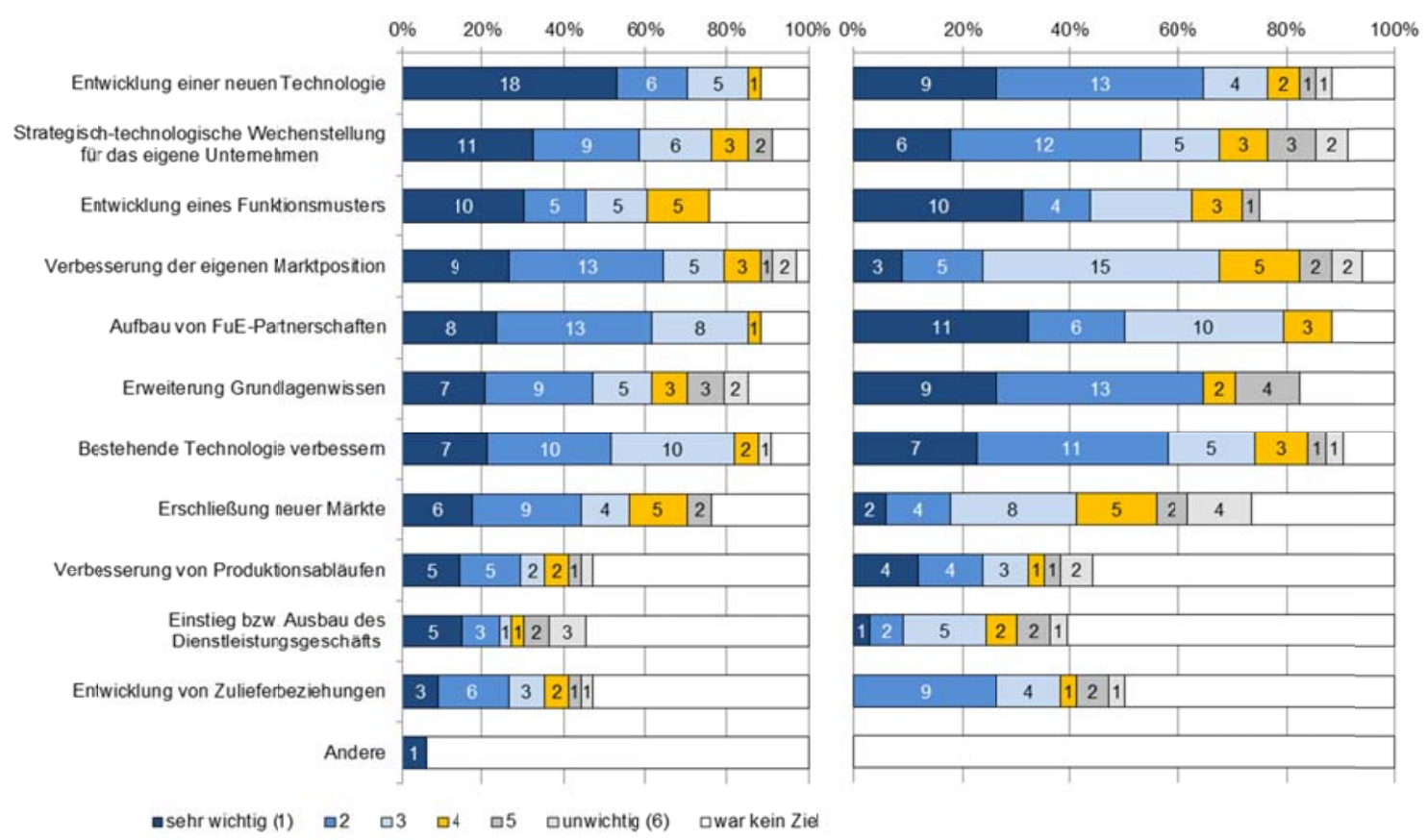

Quelle: Befragung von Unternehmen, $n=34$

Die Forschungseinrichtungen zeichnen in puncto Zielerreichung ein ähnliches Bild wie die Unternehmen. Eine leichte Abweichung der Zielerreichung findet sich auch hier bei den Technologieentwicklungszielen (Entwicklung einer neuen Technologie bzw. Komponenten und Entwicklung eines Funktionsmusters). Auch gelingt es innen weniger stark als gewünscht, neue Aufträge aus der Wirtschaft zu generieren. Besser als erwartet gelang jedoch die Schaffung von Möglichkeiten für Studienabschlussarbeiten sowie die Positionierung innerhalb der Scientific Community. Diese zwei Aspekte sind insofern bemerkenswert, als dass sie einen Effekt beschreiben, der innerhalb des Wissenschaftssystems erzielt wurde, während das Programmdesign stärker auf die Kooperation von Wirtschaft und Wissenschaft ausgelegt ist. 
Grafik 15 Welche Projektziele wurden verfolgt bzw. erreicht (FE)

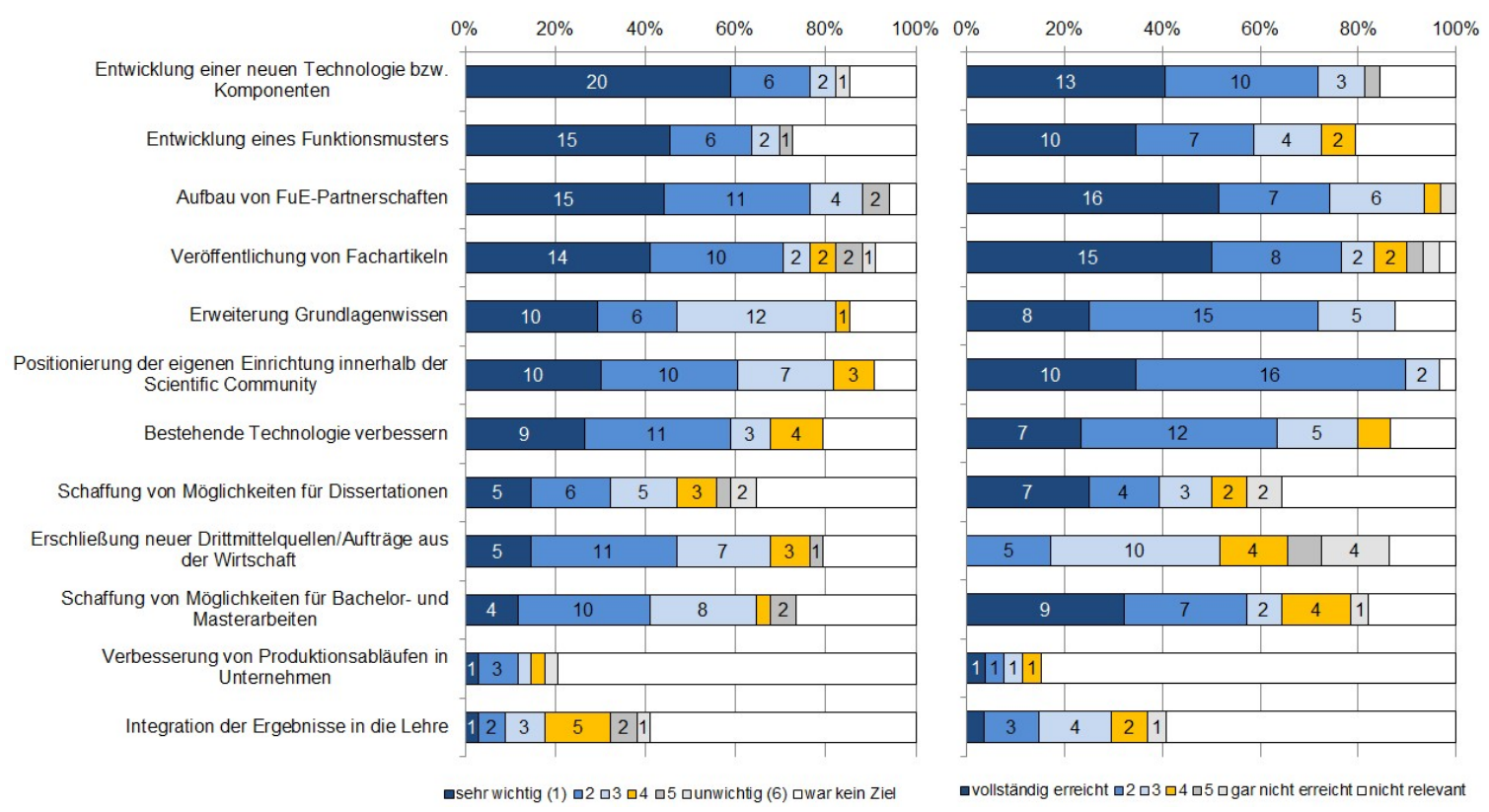

Quelle: Befragung von Forschungseinrichtungen, $n=34$

\section{Beiträge der Projekte zu den Programmzielen}

Die in Unternehmen durchgeführten Projekte leisten den stärksten Beitrag zu dem Programmziel, kooperative Forschungsprojekte zu intensivieren. Da die Projekte alle als kooperative Projekte durchgeführt werden, überrascht es nicht, dass die höchste Zahl der Antwortenden hier einen Beitrag bestätigen kann. Es folgen gleichauf die Ziele Vernetzung der FuE-Community, Sicherung der Wettbewerbsfähigkeit und Verbesserung der Sichtbarkeit über die Landesgrenzen hinaus. Die grundlegenden Ziele, die generell mit Forschungsförderungsprogrammen angestrebt werden, werden durch die geförderten Projekte damit gut unterstützt.

Weniger als ein Fünftel der Projekte sieht einen relativ hohen Beitrag des eigenen Projekts zum Programmziel ,Komfort im Lufttransportsystem verbessern‘. Dieses Ziel wird im Programmdokument zusammengefasst mit der Verbesserung der Effizienz, der Sicherheit und der Klimaschutzorientierung des Lufttransportsystems formuliert, und fällt insbesondere im Vergleich zu Sicherheit und Effizienz ab. Damit stellt sich die Frage, inwiefern eine Verbesserung des Komforts für sich genommen eine Rechtfertigung als eigens angeführtes Programmziel hat. An zweiter Stelle der umgekehrten Betrachtung folgt die Klimaschutzorientierung: Mehr als ein Drittel der in Unternehmen durchgeführten Projekte leisteten demnach keinen Beitrag zur Programmzielerreichung. Zusätzlich ist dies das einzige Programmziel, zu dem keiner der Antwortenden sehr starke Beiträge seines Projektes sieht. Aufgrund der Wichtigkeit des übergeordneten Programmziels, die Doppeldividende aus Sicherung des 
Mobilitätsbedarfs und Klimaschutz erreichen zu wollen, wäre es zu erwarten gewesen, dass mitunter Projekte stärkere Beiträge zu diesem Programmziel berichtet würden. Dies, zusammen mit einigen Aussagen aus den Interviews, legt die Interpretation nahe, dass der gesellschaftliche Nutzen zu Gunsten der wirtschaftlichen Ziele im Zeitverlauf etwas in den Hintergrund gedrängt wurde. Es sollte dabei nicht übersehen werden, dass Effizienzgewinne im Luftverkehr durch Gewichtsreduktion in der Regel auch dem Klimaschutz zu Gute kommen; sie stellen aber nur einen (kleineren) Teil der Antwort auf die Klimaschutzfrage durch den Luftverkehr dar.

Grafik 16 Programmziele, die durch die Projektergebnisse unterstützt werden konnten (Unternehmen)

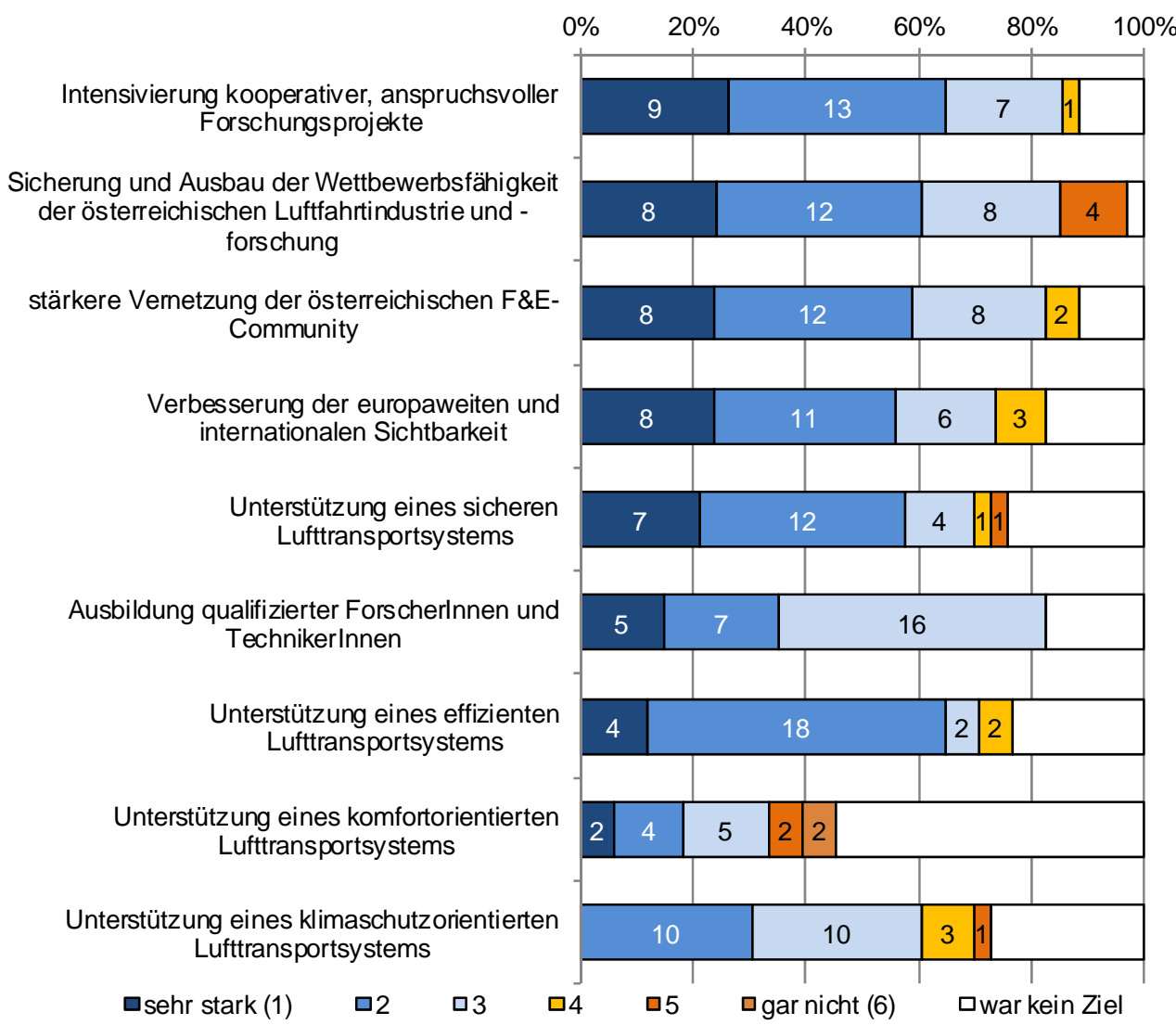

Quelle: Befragung von Unternehmen, $n=33$ 
Die Angaben der Forschungseinrichtungen zeichnen ein vergleichbares Bild. Auch hier wird von den wenigsten Projekten ein Beitrag zu Komfortorientierung und zum Klimaschutz geleistet (die Überlappung der Projekte ist relativ gering).

\section{Grafik 17 Programmziele, die durch die Projektergebnisse unterstützt werden konn-} ten (FE)

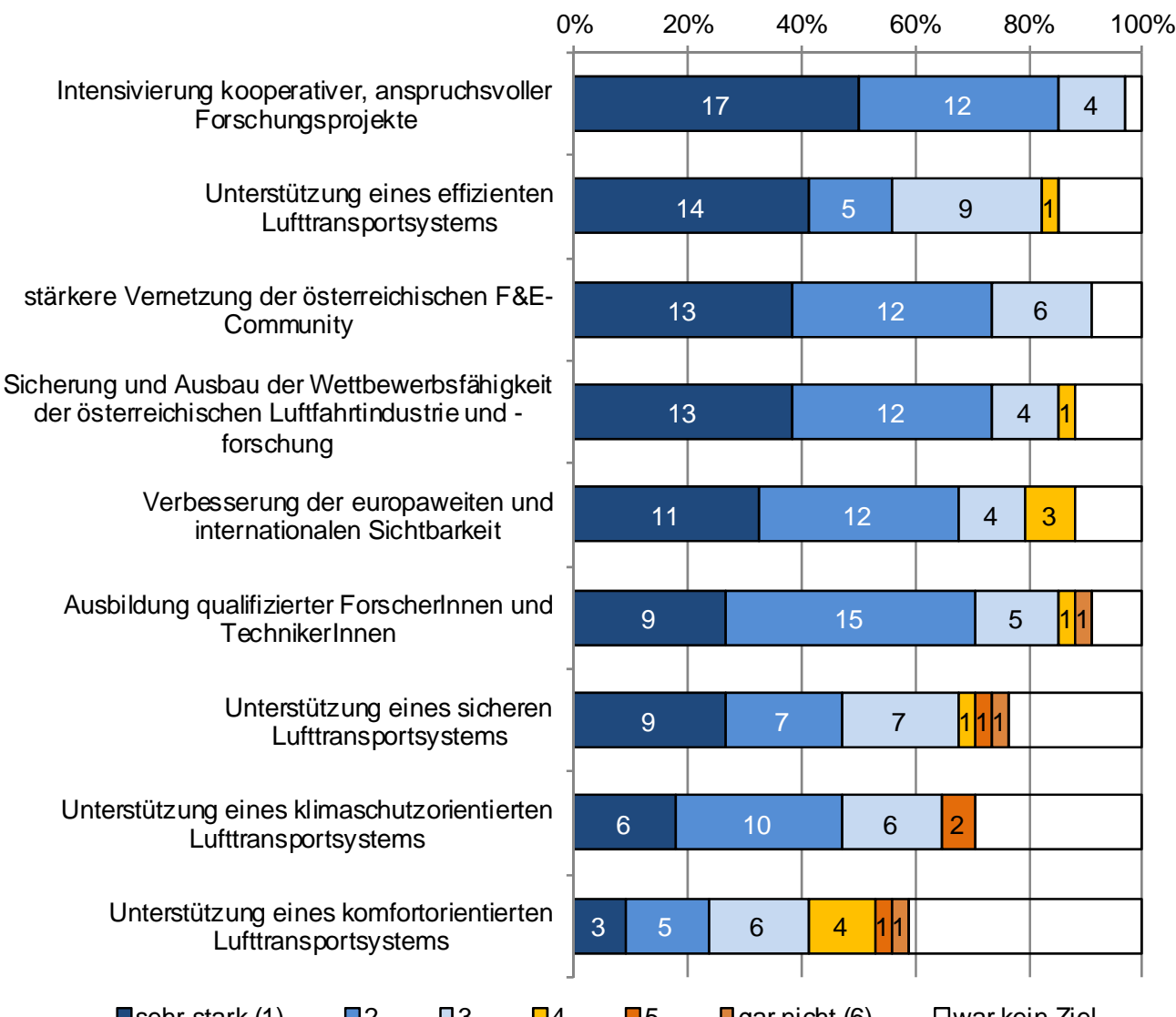

Quelle: Befragung von Forschungseinrichtungen, $n=34$ 


\section{Weiterentwicklung von Technologien}

In der schriftlichen Befragung wurde darum gebeten, die Technologiereifegrade (Technology Readiness Level, TRL) zu Beginn der geförderten Projekte, das angestrebte TRL sowie den zum Projektende erzielten Technologiereifegrad anzugeben. Dazu wurden im Fragebogen ausführliche Definitionen ${ }^{3}$ für die unterschiedlichen TRL zur Auswahl angeboten.

Die Technologiereife zu Beginn der geförderten Projekte liegt für die Unternehmen im Schnitt etwas höher als für die Forschungseinrichtungen. Dies ist ein erwartbares Resultat, da Unternehmen eher an der (Weiter-)Entwicklung von Technologien orientiert sind, während Forschungseinrichtungen sich eher der Forschung widmen. Die meisten der in den Unternehmen angestrebten Technologiereifegrade befinden sich auf TRL 4 (vgl. Grafik 18). Dies bedeutet, dass in der Regel die Weiterentwicklung über zwei Levels angestrebt wird. Dies entspricht einer Weiterentwicklung einer Technologie, wie sie in der Regel im Rahmen eines geförderten Projektes erreicht werden kann. Interessanterweise gibt es einige Projekte, die höhere TRL (7 bis 9) anstrebten. Dies ist vorerst etwas erstaunlich, da im Rahmen von Take Off Projekte mit einem TRL 2 bis $6^{4}$ gefördert werden sollen.

Hierbei sind zwei Aspekte zu beachten: Erstens haben Teilnehmer an einem Projekt die Klassifikation für ihre jeweiligen Projektteile unterschiedlich vorgenommen: Koordinatoren hatten das Gesamtprojekt beurteilt, während andere Projektteilnehmer ihre Projektteile im Fokus hatten. Letztere können, individuell beurteilt, durchaus bereits näher an der Anwendung liegen. Darüber hinaus gibt es in der Luftfahrt fallweise aufwändige Demonstratorentwicklungen (TRL 6-8), die eine öffentliche Förderung durchaus rechtfertigen können. Angeschrieben waren jeweils die für die Teilprojekte verantwortlichen Ansprechpartner in den Unternehmen bzw. Forschungseinrichtungen. Diese haben auch die Fragebögen beantwortet. Es bleibt jedoch nicht auszuschließen, dass in Einzelfällen trotz der genauen Definition im Fragebogen der Einschätzung der TRL ein unterschiedliches Verständnis zugrunde liegt. Insgesamt ist jedoch die Verwendung des Konzepts der Technologiereifegrade in der Luftfahrtbranche sehr verbreitet, so dass von einer relativ einheitlichen Verwendung der Begriffe ausgegangen werden kann. Das primäre Interesse dieser Klassifikation ist jedoch nicht, eine ganz korrekte Klassifikation von Gesamt-Projekten zu erstellen, sondern zu eruieren, ob weitere Schritte in der Entwicklung getätigt wurden.

${ }^{3}$ Für jedes Technology Readiness Level 1 bis 9 wurden die Definitionen für flugzeugspezifische Hardware (NASA/ ESA-Definition), die allgemeine Beschreibung (Definition des US-Department of Defence) sowie die OECD-Definition (Frascati Handbuch) nebeneinandergestellt, um eine Zuordnung auch für unterschiedliche Projekttypen möglich zu machen.

${ }^{4}$ bmvit Eigendarstellung (siehe Anhang). 


\section{Grafik 18 Technologiereifegrade vor Projektbeginn, angestrebt und tatsächlich} erreicht (Unternehmen)

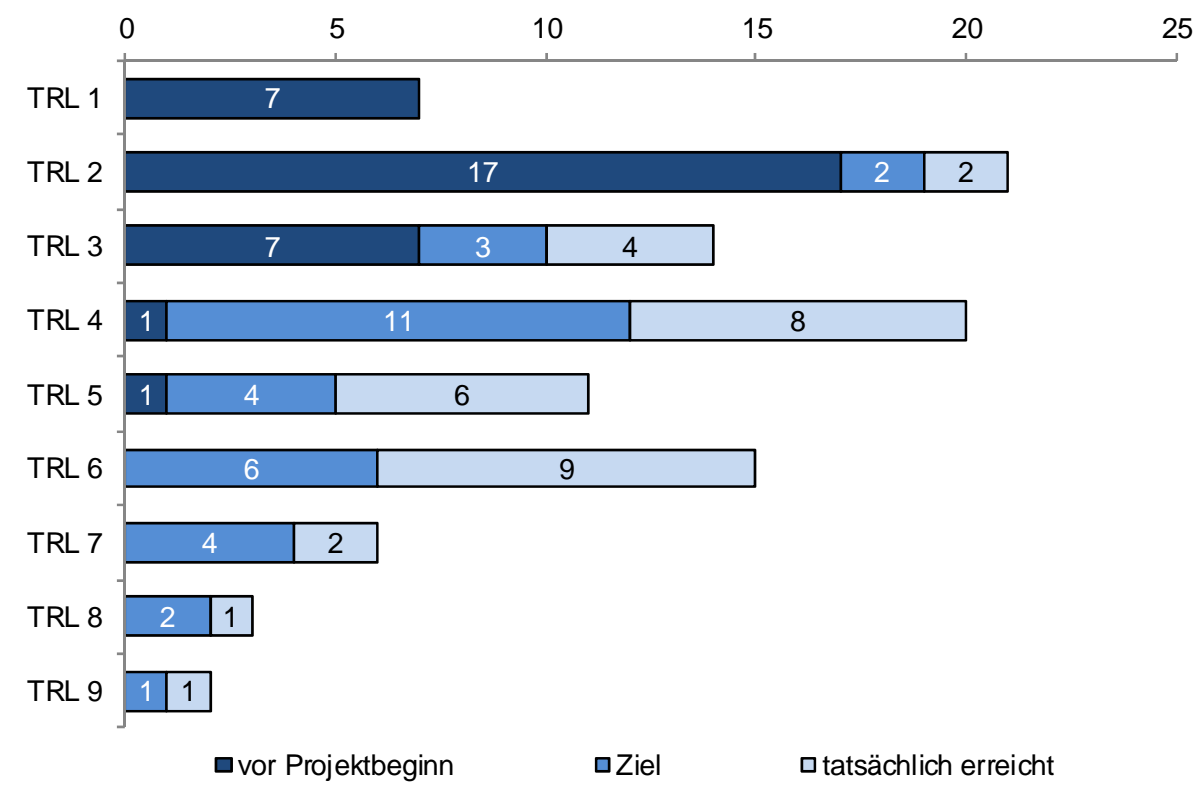

Quelle: Befragung von Unternehmen, $n=33$

Bei den Forschungseinrichtungen beginnt die Mehrzahl der Projekte auf den TRL 1 und TRL 2, wobei sich zeigte, dass die TRL 1 Bewertung wiederum auf individuelle Projektteile basiert. Gleichzeitig zeigt sich aber auch, dass die FuE-Tätigkeiten der FE in einem relativ höheren Ausmaß von einem höheren TRL ausgegangen sind. Verglichen mit den Unternehmen liegt das angestrebte TRL allerdings überwiegend im Bereich der TRL 3 bis 5. Das bedeutet, dass die Zielsetzung der FE mehrheitlich die im Take Off Programm anvisierten Technologiereifegrade (OECD: TRL 2 bis 6 ) anstrebt, während die Unternehmen häufiger auch in den Bereich der experimentellen Entwicklung (OECD: TRL 5 bis 8) vorstoßen wollten. 
Grafik 19 Technologiereifegrade vor Projektbeginn, angestrebt und tatsächlich erreicht (Forschungseinrichtungen)

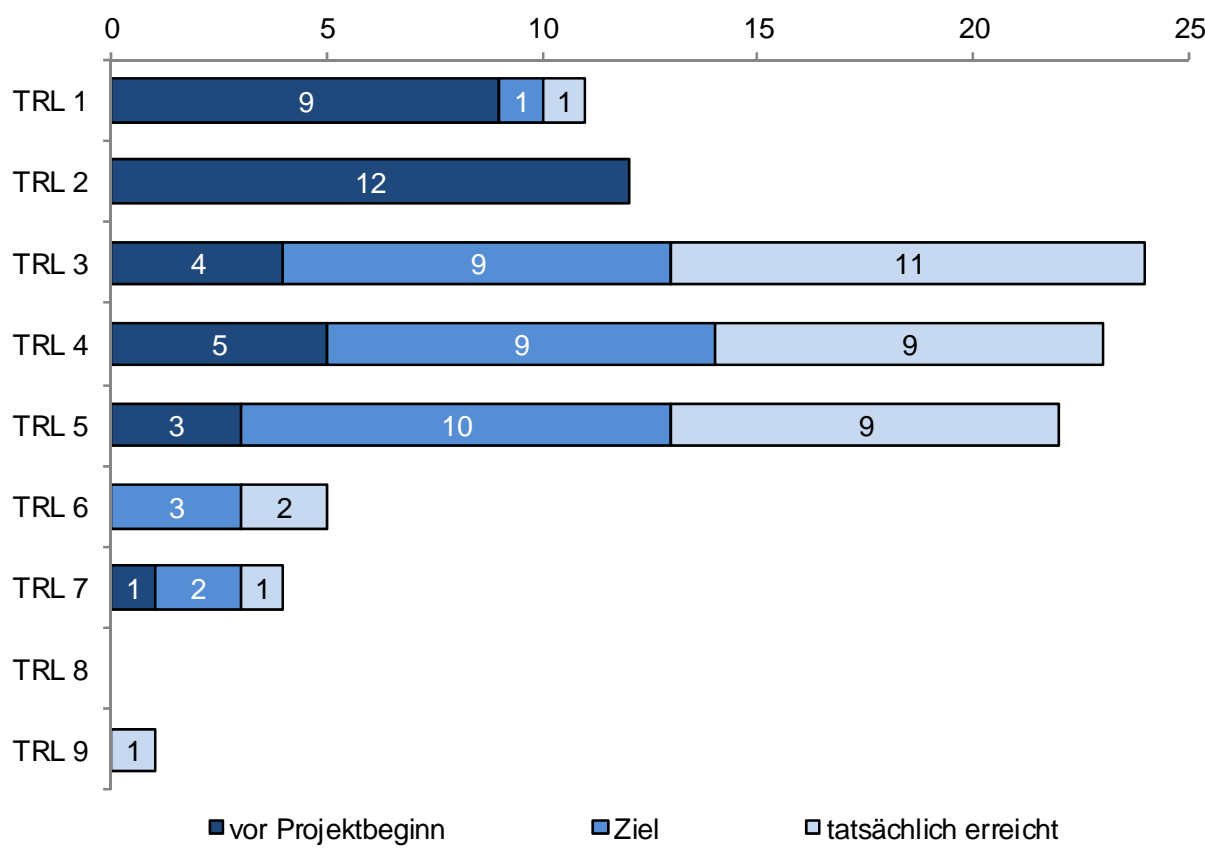

Quelle: Befragung von Forschungseinrichtungen, n=34; z.B. wurde für die Angabe für den tatsächlich erreichten TRL 9 innerhalb eines Projektteiles eine bestehende Technologie für einen anderen Anwendungsbereich für die Luftfahrt adaptiert. Dies sagt aber noch nichts über das TRL Niveau des Gesamtprojektes aus.

Die Betrachtung der Differenzdarstellung zeigt, wie viele der Projekte die angestrebten Technologiereifegrade in der Projektlaufzeit erreichen konnten. Dargestellt ist die Differenz zwischen den angestrebten und den tatsächlich zu Ende der Förderdauer erreichten Technologiereifegraden. Negative Werte geben an, um wie viele TRL das Projektziel verfehlt wurde. Eine Null bedeutet, dass das angestrebte TRL mit dem Projektende erreicht wurde und positive Werte zeigen, dass höhere TRL mit dem Projekt erzielt werden konnten als ursprünglich geplant. Eine Forschungseinrichtung und neun Unternehmen geben an, dass sie die angestrebten Ziele in Bezug auf die Weiterentwicklung der Technologie nicht erreichen konnten. Dabei haben die meisten Antwortenden ihr angestrebtes Ziel um ein TRL verfehlt. Die überwiegende Mehrheit aller Antwortenden (67\%) geben an, innerhalb der Projektlaufzeit das angestrebte TRL erreichen konnten und immerhin $12 \%$ haben ihre ursprünglich gesetzten Ziele um ein bis drei Technologiereifegrade übertroffen. Letztere Angaben stammen alle von Unternehmen. 


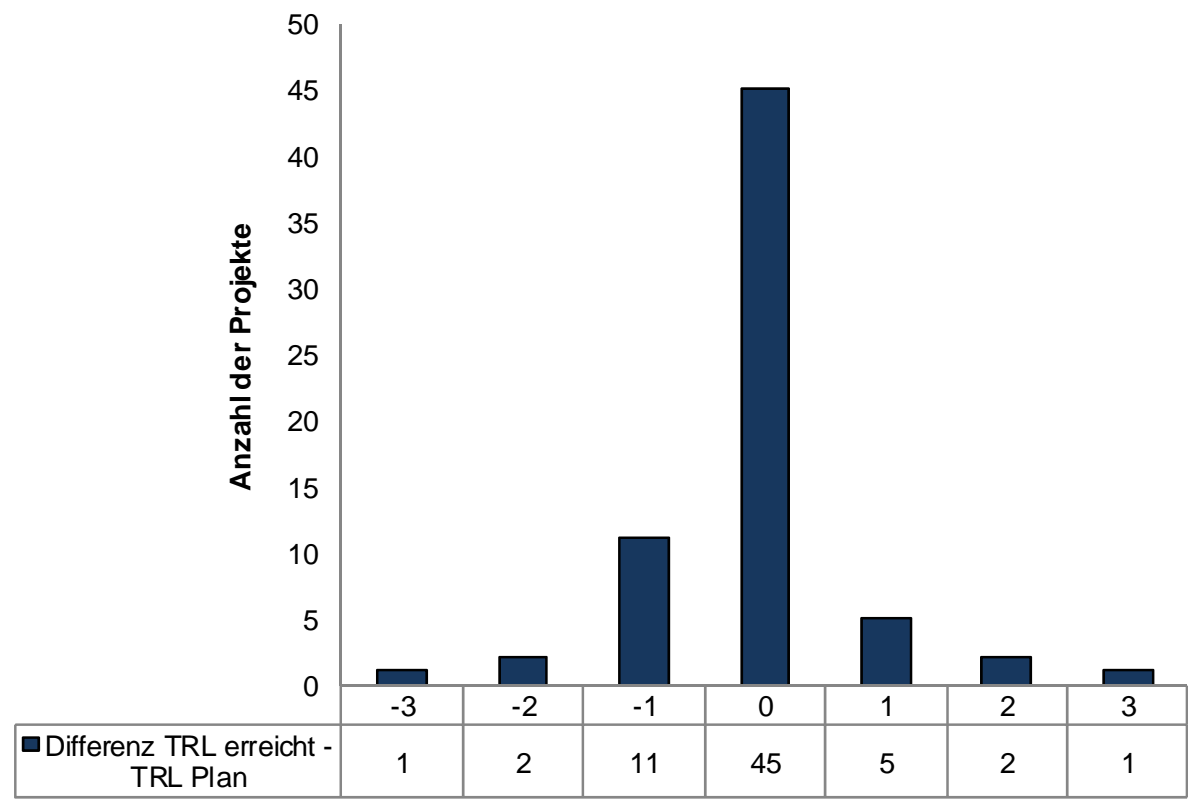

Quelle: Befragung von Unternehmen und Forschungseinheiten, $n=67$

\section{Verbreitung der Projektergebnisse in der Fachöffentlichkeit}

Nur ein Drittel (34 Prozent) der antwortenden Unternehmen (12) gibt an, überhaupt Aktivitäten zur Verbreitung des Projekts oder seiner Ergebnisse in der Fachöffentlichkeit unternommen zu haben. Für ein öffentliches Förderprogramm im vorwettbewerblichen Bereich, das vom Transfer der Ergebnisse profitieren soll, ist dieser Wert zu niedrig. Zum Vergleich: Bei der Evaluation des deutschen Luftfahrtforschungsprogramms hat sich gezeigt, dass für LuFo III rund $57 \%$ der befragten Verbundvorhaben in Unternehmen angaben, Fachaufsätze und/oder wissenschaftliche Publikationen hervorgebracht zu haben. Für LuFo IV lag dieser Wert auch nur noch bei $37 \%$ (vgl. Groß/Kerlen 2013, S. 47). Jedes dritte Unternehmen hat demnach an einer der von Take Off veranstalteten Vernetzungsveranstaltungen teilgenommen, einem relativ leicht wahrzunehmenden Angebot, um den Transfer zu fördern und die Vernetzung zu stärken. Ein Unternehmen weist in der schriftlichen Befragung explizit auf den Nutzen dieser Transferveranstaltungen hin, um weitere Projekte zu initiieren. 
Tabelle 5 Aktivitäten von Unternehmen zur Verbreitung des Projekts und der Projektergebnisse in der Fachöffentlichkeit

\begin{tabular}{|l|c|c|c|}
\hline & $\begin{array}{c}\text { Anzahl der } \\
\text { Projekte mit } \\
\text { Aktivitäten }\end{array}$ & $\begin{array}{c}\text { Summe der } \\
\text { Aktivitäten }\end{array}$ & $\begin{array}{c}\text { Mittelwert für } \\
\text { alle Projekte }\end{array}$ \\
\hline $\begin{array}{l}\text { Fachaufsätze/wissenschaftliche Pub- } \\
\text { likationen }\end{array}$ & 12 & 28 & 0,8 \\
\hline $\begin{array}{l}\text { Publikationen zu den Projektergeb- } \\
\text { nissen in (praxisorientierten) Bran- } \\
\text { chenzeitschriften }\end{array}$ & 7 & 10 & 0,3 \\
\hline $\begin{array}{l}\text { Vorträge auf nationalen und internati- } \\
\text { onalen Fachtagungen oder Work- } \\
\text { shops (ohne Publikation) }\end{array}$ & 11 & 26 & 0,8 \\
\hline Messeauftritte & 5 & 11 & 0,3 \\
\hline $\begin{array}{l}\text { Teilnahme an von Take Off veranstal- } \\
\text { teten Transfer-Workshops }\end{array}$ & 10 & 1 & 0,4 \\
\hline $\begin{array}{l}\text { Weiteres } \\
\text { Interne Berichterstattung über } \\
\text { den Projektstatus (abteilungs- } \\
\text { übergreifend) }\end{array}$ & 1 & 14 & \\
\hline
\end{tabular}

Quelle: Befragung von Unternehmen, n=34; Da es seit dem Jahr 2008 für die Take Off TransferWorkshops eine Anwesenheitspflicht zumindest eines Partners pro Projekt gibt, scheinen die angegebenen 10 vertretenen Projektteilnehmer als (zu) niedrig. Hier könnten andere Personen innerhalb eines Unternehmens teilgenommen haben.

Bei den Forschungseinrichtungen liegt der Wert mit zwei Dritteln (21), die das Projekt oder die Projektergebnisse in der Fachöffentlichkeit verbreitet haben, zwar höher als bei den Unternehmen. Hier wäre jedoch zu erwarten, dass jede Forschungseinrichtung Aktivitäten zur Verbreitung seiner Arbeit in der Fachöffentlichkeit unternimmt, da Publizieren eines der Hauptziele wissenschaftlicher Tätigkeit ist. Hier könnte es mitunter Regelungen zwischen den Unternehmen und den Forschungseinrichtungen gegeben haben, die es den Forschungseinrichtungen untersagen, über die Projektergebnisse zu berichten, da mögliche wirtschaftlichen Nachteile bei den kooperierenden Unternehmen befürchtet werden. 
Tabelle 6 Aktivitäten der Forschungseinheiten zur Verbreitung des Projekts und der Projektergebnisse in der Fachöffentlichkeit

\begin{tabular}{|l|c|c|c|}
\hline & $\begin{array}{c}\text { Anzahl der } \\
\text { Projekte mit } \\
\text { Aktivitäten }\end{array}$ & $\begin{array}{c}\text { Summe der } \\
\text { Aktivitäten }\end{array}$ & $\begin{array}{c}\text { Mittelwert für } \\
\text { alle Projekte }\end{array}$ \\
\hline $\begin{array}{l}\text { Fachaufsätze/ wissenschaftliche } \\
\text { Publikationen }\end{array}$ & 21 & 58 & 1,7 \\
\hline $\begin{array}{l}\text { Publikationen zu den Projektergeb- } \\
\text { nissen in (praxisorientierten) Bran- } \\
\text { chenzeitschriften }\end{array}$ & 6 & 16 & 0,5 \\
\hline $\begin{array}{l}\text { Vorträge auf nationalen und internati- } \\
\text { onalen Fachtagungen oder Work- } \\
\text { shops (ohne Publikation) }\end{array}$ & 25 & 51 & 1,5 \\
\hline Messeauftritte & 21 & 10 & 0,3 \\
\hline $\begin{array}{l}\text { Teilnahme an von Take Off veranstal- } \\
\text { teten Transfer-Workshops }\end{array}$ & 5 & 4 & 1,1 \\
\hline $\begin{array}{l}\text { Weiteres } \\
\text { Bilaterale Workshops mit mögli- } \\
\text { chen Anwendern }\end{array}$ & & 2 & 1 \\
\hline $\begin{array}{l}\text { Projektvorstellungen bei potenti- } \\
\text { ellen Partnern/Kunden }\end{array}$ & $\begin{array}{l}\text { Radiobeitrag bei Ö1 - Digital } \\
\text { Leben }\end{array}$ & 1 & \\
\hline \begin{tabular}{l} 
Teilnahme Summer School \\
\hline
\end{tabular}
\end{tabular}

Quelle: Befragung von Forschungseinrichtungen, $n=34$

Aus einer gesamtwirtschaftlichen Perspektive betrachtet ist der Vorteil von Aktivitäten zur Verbreitung der Projektergebnisse darin zu sehen, dass diese Wissenstransfers ermöglichen und damit Voraussetzung für einen brancheninternen, aber auch einen branchenübergreifenden Wissens- und Technologie Spillover sind. Dies gilt für Vorträge/Messeauftritte ebenso wie für Publikationen. Fachpublikationen sind jedoch gegenüber einer nicht-schriftlichen Form der Verbreitung insofern vorzuziehen, als dass sie auch für weitere Nutzerinnen und Nutzer jenseits der bei einer Veranstaltung anwesenden verfügbar sind. Vor diesem Hintergrund ist der Anteil der Publikationen, der aus den im Rahmen von Take Off geförderten Projekten hervorgeht, als relativ gering zu beurteilen.

$19 \%$ der befragten Forschungseinrichtungen und $6 \%$ der Unternehmen haben in Folge des Take Off-Projektes gewerbliche Schutzrechte eingereicht. Insbesondere die Rate in Unternehmen ist im Vergleich mit anderen Luftfahrtforschungsprogrammen als gering einzustufen. Insgesamt sind 10 Schutzrechte von Forschungseinrichtungen angemeldet worden, weitere vier von Unternehmen und zwei von anderen Einrichtungen. Erstaunlich ist zusätzlich, dass Forschungseinrichtungen mehr Schutzrechte angemeldet haben als Unternehmen, da bei ersteren die Erteilung und Anwendung von Patenten aufgrund der nicht-wirtschaftlichen Ausrichtung in der Regel eine geringere Rolle spielt. 


\section{Qualifizierung}

Insgesamt 18 Dissertationen wurden in den Unternehmen (10) und Forschungseinrichtungen (8), die an der Befragung teilgenommen haben, angefertigt. Dies bedeutet, dass in knapp jeder vierten Organisation $(3,7)$ eine Promotion durchgeführt wurde. 35 Studienabschlussarbeiten (Diplomarbeiten, Masterarbeiten) wurden im Laufe der Projekte in den Forschungseinrichtungen (26) und Unternehmen (9) angefertigt. Damit wurde in knapp jeder zweiten durch Take Off geförderten Organisation $(1,9)$ eine Studienabschlussarbeit angefertigt. Dies ist ein erfreulicher Nebeneffekt von Take Off, da damit praxisorientierte Studienabschlüsse erfolgen, und die Absolventen damit gut für den Eintritt in den Arbeitsmarkt vorbereitet sind.

\subsection{Aufwendungen und Begleitmaßnahmen}

Als Aufwendungen zur Unterstützung der Marktentwicklung wurden im Rahmen von Take Off eine Reihe von Studien erstellt, die sich z.B. mit der Positionierung der Luftfahrtunternehmen in den Wertschöpfungsketten, der Innovations- und Kooperationspotenziale der österreichischen Luftfahrtindustrie, oder dem Risikomanagement der Luftfahrtunternehmen widmen. ${ }^{5}$ Diese Themen scheinen für die Entwicklung einer dynamischen, und in Österreich relativ jungen, Branche sehr relevant.

Darüber hinaus sind unter Begleitmaßnahmen vornehmlich die Vernetzungsaktivitäten durch das Programmmanagement subsumiert, die auf die Etablierung von tragfähigen Kooperationen zwischen Unternehmen und Forschungseinheiten abzielten. Davon wurde auch vermehrt Gebrauch gemacht, z.B. mit unterschiedlichen Formaten der Vernetzung erfolgreich experimentiert. Die ab einem späteren Zeitpunkt eingeführte vertragliche Verpflichtung von Fördernehmern sich an Vernetzungsaktivitäten beteiligen zu müssen, wird angesichts der Entwicklungsphase des österreichischen Marktes als sehr sinnvoll eingeschätzt.

Gemäß Programmdokument sollten für Begleitmaßnahmen maximal $10 \%$ des Budgets verwendet werden, das aufgrund des starken Informationsbedarfs für die noch junge Branche sowie des hohen Vernetzungsbedarfs der Unternehmen und Forschungseinrichtungen durchaus gerechtfertigt sein kann. Der realisierte Umfang der Begleitmaßnahmen liegt bei rd. € 440,000 im Zeitraum 2002 - 2013 und ist damit im Verhältnis zum Gesamtbudget (€ 65,7 Mio.) durchaus verhältnismäßig.

${ }^{5}$ http://www.bmvit.gv.at/innovation/luftfahrt/studien/index.html 


\section{Ergebnisse (Outcomes) und Wirkungen (Im- pacts)}

\subsection{Unmittelbare Ergebnisse bei der Zielgruppe}

\section{Nutzen der Programmteilnahme}

Die für die Zielgruppe angestrebten Ziele der Technologieentwicklung und der Erweiterung des technologischen Wissensfundus werden gut erreicht. Für die meisten Unternehmen liegt der Nutzen der Teilnahme an Take Off in der Entwicklung eines neuen Projekts (44\%), gefolgt von der Entwicklung eines neuen Verfahrens zur Herstellung der Produkte/Dienstleistungen. Damit sind die Voraussetzungen für eine potenzielle wirtschaftliche Verwertung der Projektergebnisse gut, da neue Produkte Marktpotenziale erschließen können.

\section{Grafik 21 Nutzen des Projekts für Unternehmen}

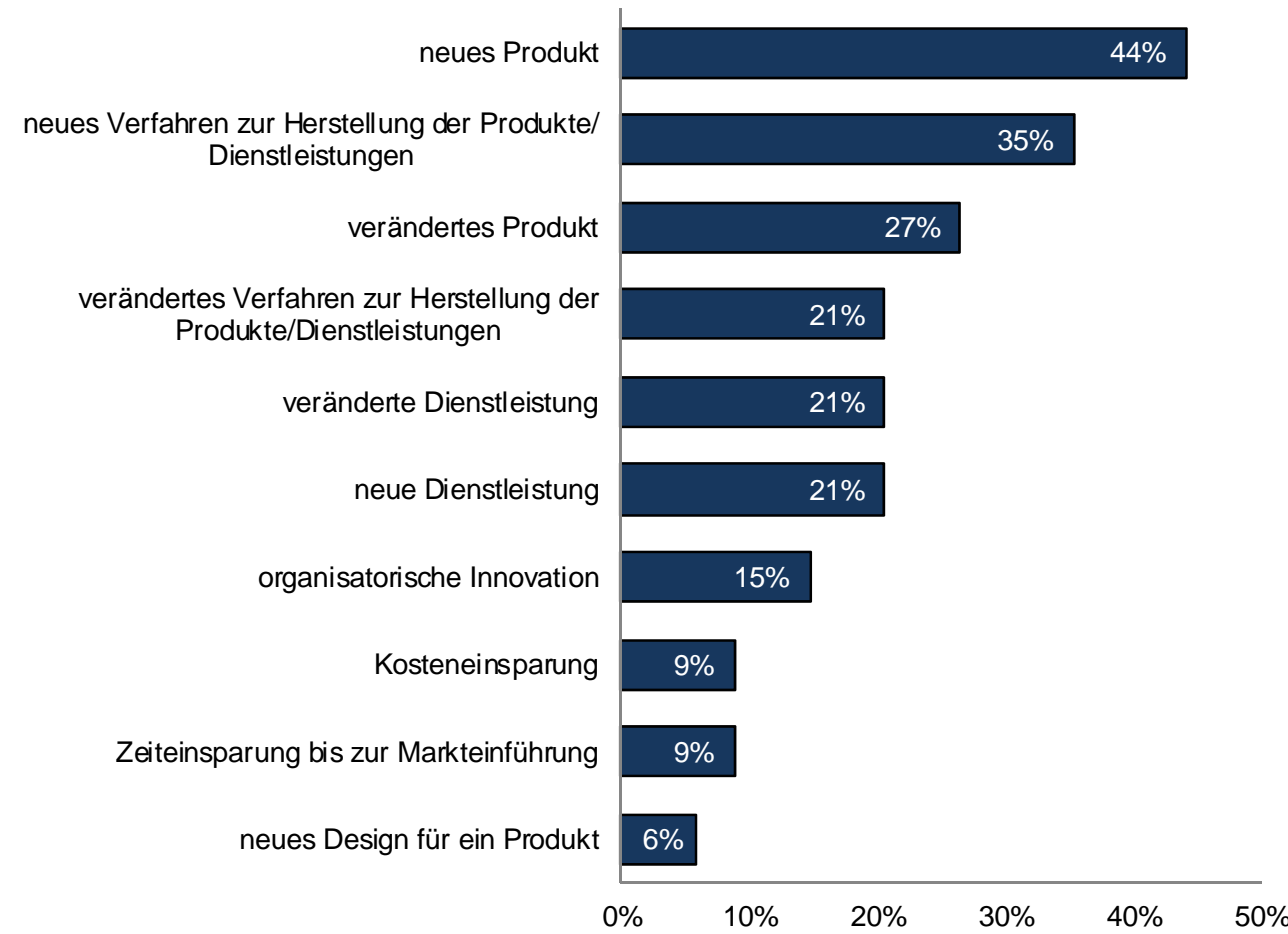

Quelle: Befragung von Unternehmen, $\mathrm{n}=33$; Reihung der Nutzen durch die Unternehmen 
Die Forschungseinrichtungen sehen mit Abstand den wichtigsten Nutzen der Teilnahme an Take Off in der Erweiterung ihrer wissenschaftlich-technologischen Kenntnisse. Knapp 80 \% der antwortenden FuE-Einrichtungen messen der Erweiterung der Wissensbasis den höchsten Nutzen bei. Weitere rund $60 \%$ der Antwortenden sehen in der Möglichkeit von Folgeprojekten in dem thematischen Fokus bzw. der Drittmittelakquisition als Finanzierungsstrategie einen weiteren hohen Nutzen von Take Off. Überwiegend mittlerer Nutzen steht in Zusammenhang mit der Publikation von akademischen Artikeln und Beiträgen zu Fachkongressen. Eher geringer bzw. gar kein Nutzen kann von den antwortenden Forschungseinrichtungen für die eigene Lehre und die Erteilung von Patenten und anderen Schutzrechten gewonnen werden.

\section{Grafik 22 Nutzen des Projektes für Forschungseinrichtungen}

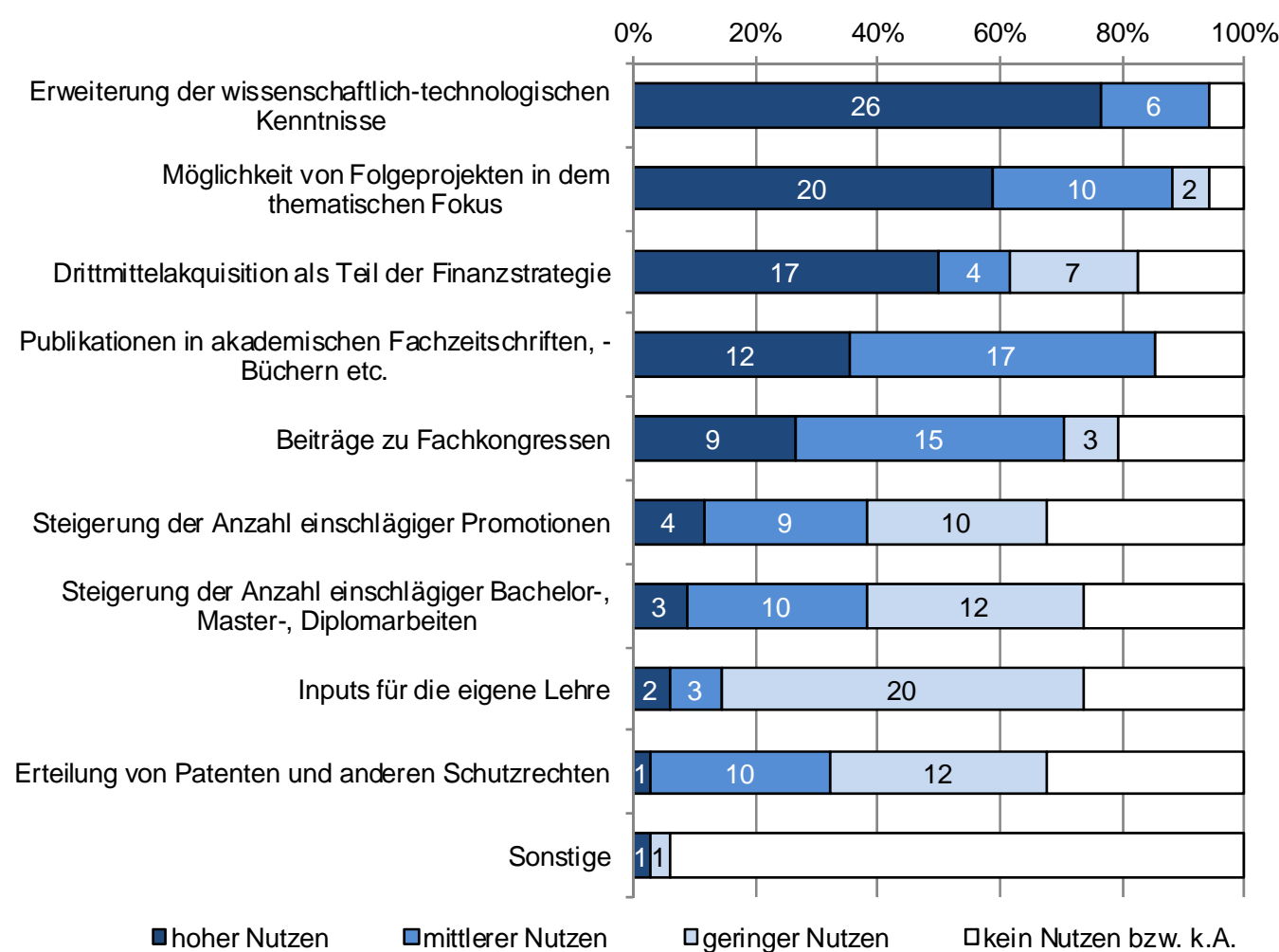

Quelle: Befragung von Forschungseinheiten, $n=33$, Mehrfachangaben zulässig 


\section{Weiterentwicklung der Technologien nach Projektende}

$57 \%$ aller Antwortenden aus Unternehmen geben an, dass ihre Projekte entweder in anderen Förderprojekten weiter verfolgt wurden, oder aber - weniger häufig - mit eigenen Mitteln (vgl. hierzu Grafik 24). 59 \% von diesen Projekten befinden sich heute auf einem höheren TRL als zum Zeitpunkt der Beendigung des Projekts. In Grafik 23 ist zum einen die Differenz zwischen den angestrebten und den tatsächlich zu Ende der Förderdauer erreichten Technologiereifegraden dargestellt (dunkelblau, siehe ebenfalls Grafik 20), zum anderen die Differenz zwischen dem zum Befragungszeitpunkt erreichten TRL und den angestrebten Technologiereifegraden (hellblau). Positive Werte zeigen hier, um wie viele TRL die Technologie nach Ende der Förderung bis zum Befragungszeitpunkt weiterentwickelt werden konnte. Negative Werte geben an, um wie viele TRL auch zum heutigen Zeitpunkt das Projektziel verfehlt wurde. Eine ganze Reihe dieser Projekte konnte dabei die Technologiereife über zwei oder sogar drei Stufen weiterentwickeln. Die Projekte, die heute ein höheres TRL aufweisen, sind zum größten Teil dem Bereich ATM und Flughafen zuzuweisen (11 der 23 Nennungen). Die Weiterentwicklung von vorhandenen Sicherheits- und Fluginformationssystemen weist daher eine höhere Dringlichkeit bzw. Marktfähigkeit auf, als die weiteren Themenbereiche von Take Off. Dies drückt sich unter anderem auch dadurch aus, dass alle ATM-Projekte der Unternehmen nach Projektende weitergeführt wurden. Damit hat sich die mit der Verabschiedung der Österreichischen Luftfahrtstrategie einhergehende stärkere Öffnung des Programms für die Luftverkehrswirtschaft als sinnvoll erwiesen.

\section{Grafik 23 Differenz zwischen erreichtem TRL (Projektende, heute) und geplantem TRL}

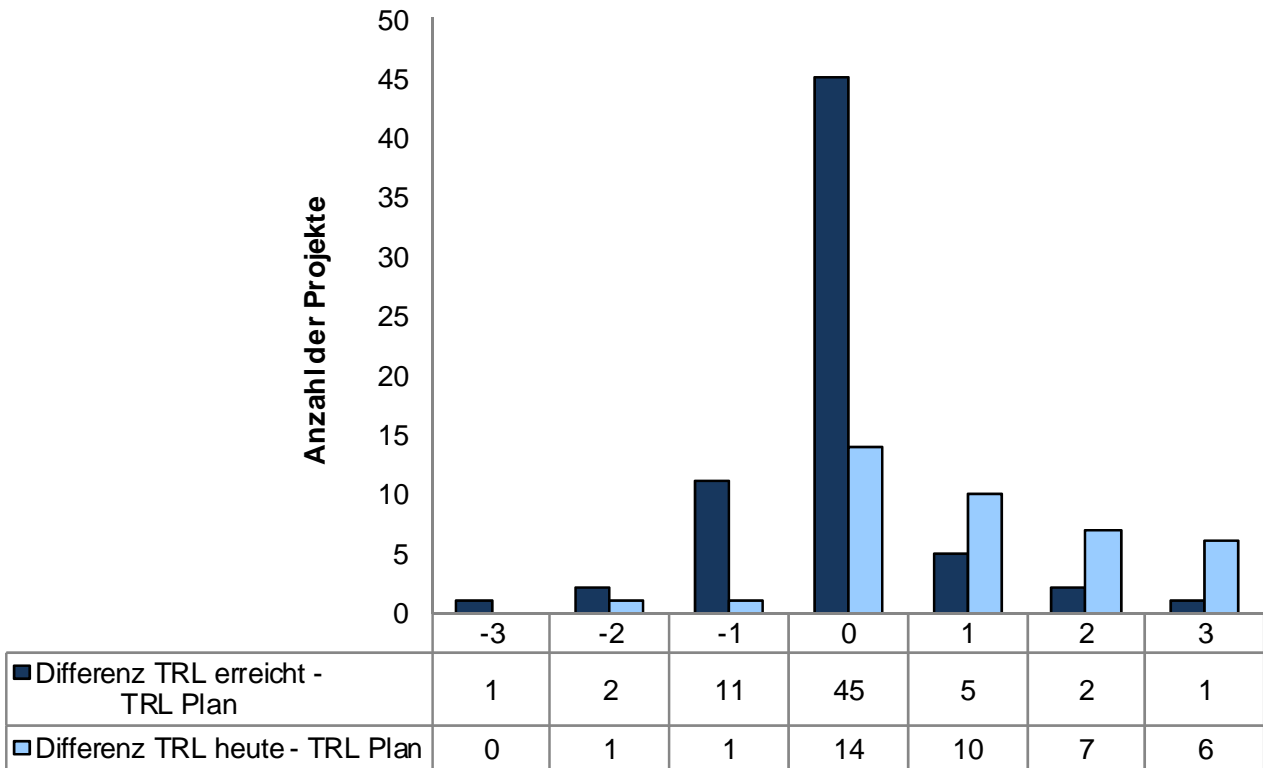

Quelle: Befragung von Unternehmen und Forschungseinheiten, $n=67$ 


\section{Grafik 24 Weiterverfolgung der Projekte nach Projektende}

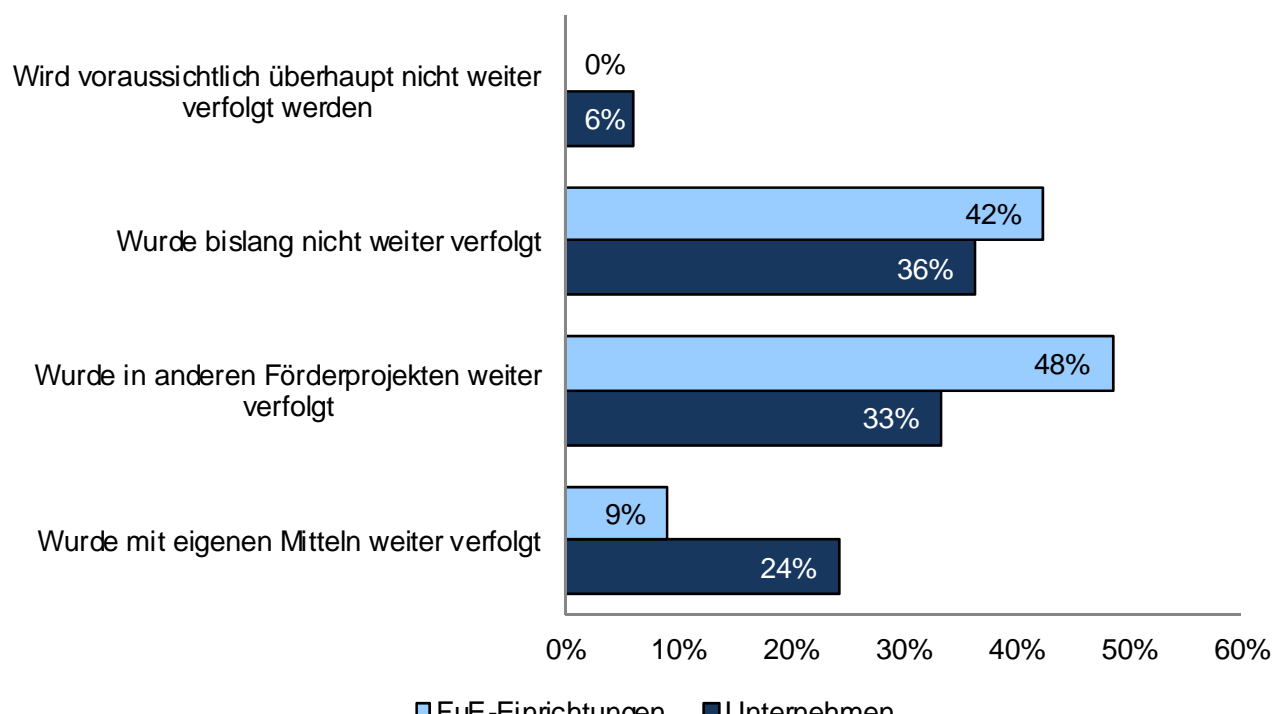

口FuE-Einrichtungen घUnternehmen

Quelle: Befragung von Unternehmen und Forschungseinheiten, n=66 (Grafik 24)

Die Tatsache, dass insgesamt 4 von 10 Unternehmensteilnehmern angeben, dass ihre Projekte gar nicht oder bislang nicht weiter verfolgt wurden, verdeutlicht das Risiko, das mit diesen geförderten Projekten verbunden ist. Die Ergebnisse dieser Projekte konnten bislang nicht derart weiterverwendet werden, dass eine absehbare Rendite erzielt werden kann. Projekte, die bislang nicht weitergeführt worden sind, können vor allem den technisch aufwendigen Bereichen zugeordnet werden. Darunter fallen etwa intelligente Fluggeräteinfrastruktur, Bodentest- und Prüfgeräte, Komplexe Flugzeugstrukturen und Bauteile, innovative Werkstoffe, Fertigungstechniken, und Triebwerke.

Dieser Schluss wird auch durch die Antworten auf die Frage nahegelegt, ob die Unternehmen das Projekt auch ohne Förderung durchführen hätten können. $86 \%$ der Projekte in Unternehmen hätten gar nicht oder nur in deutlich geringerem Ausmaß durchgeführt werden können (vgl. Grafik 25). Insbesondere die fehlende Finanzierung und das hohe Risiko werden als Gründe hierfür angeführt. Bei $71 \%$ der Take Off Unternehmen wäre es zu einer zeitlichen Verzögerung der Projektdurchführung gekommen. Eine Durchführung der Projekte ohne die gewünschten Kooperationspartner wäre für kaum einen der Projektteilnehmer die Folge gewesen, was den Wert der Kooperationsbeziehungen unterstreicht. Bei den Forschungseinrichtungen liegt der Anteil der Projekte, die gar nicht durchgeführt worden wären, naheliegender Weise noch höher. Hier stehen meist keine Mittel aus der Grundfinanzierung für Kooperationsprojekte mit Unternehmen zur Verfügung. 


\section{Grafik 25 Durchführung des Projekts ohne Förderung}

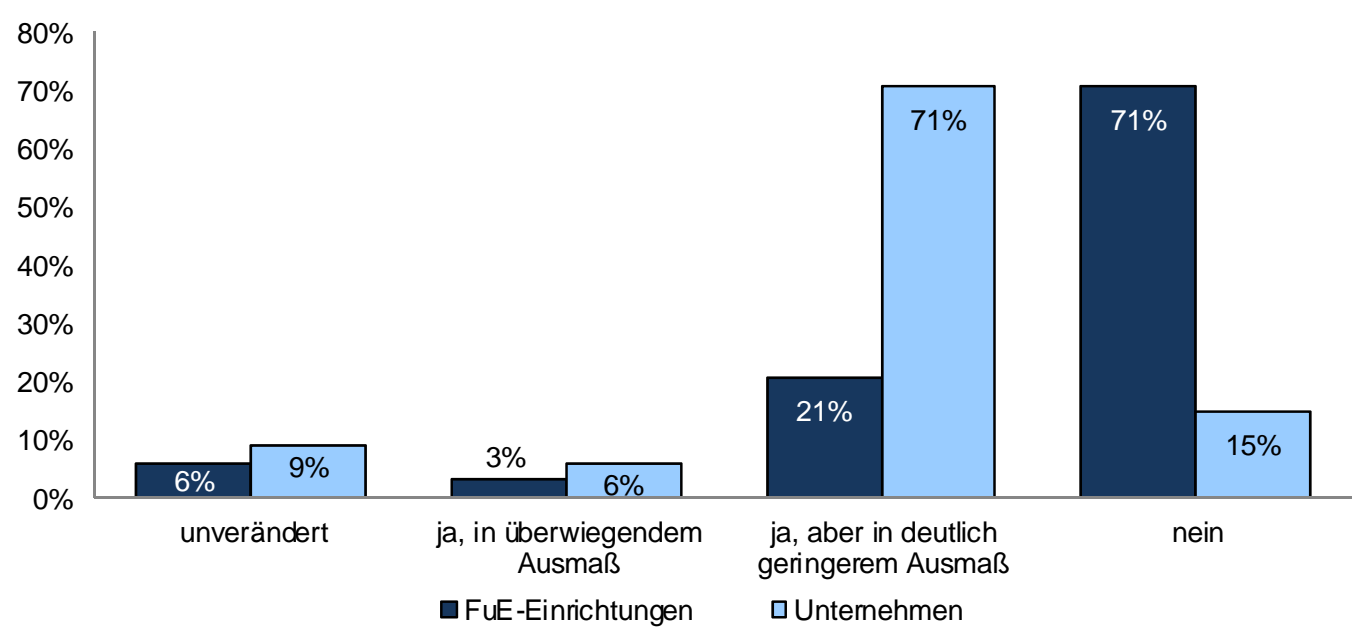

Quelle: Befragung von Unternehmen und Forschungseinheiten, $n=68$

Obige Grafik zeigt Hinweise auf das Vorliegen von Mitnahmeeffekten im beschränkten Ausmaß. Dieser Effekt sollte nicht überbewertet werden, da diese Projekte lediglich 5,8 \% (Unternehmen) bzw. 6,6 \% (Forschungseinrichtungen) der Fördersumme der Antwortenden ausmachen. Insbesondere bei FE kann man vereinzelt beobachten, dass aus Eigeninteresse mancher (ausfinanzierter) Forscher auch ohne Förderung teilgenommen hätte. Zum Vergleich: Im Zuge des FFG-Wirkungsmonitoring 2013 haben 19 \% der befragten Unternehmen angegeben, dass die Forschungsprojekte in unverändertem oder überwiegendem Ausmaß durchgeführt worden wären. Dieser etwas höhere Wert lässt sich auf die vergleichsweise geringere Marktentfernung der Basisprogramm-Projekte zurückführen.

Bei der Zwischenevaluierung von Take Off gaben nur 2,6 \% der Unternehmen - im Vergleich zu $9 \%$ - an, dass das Projekt ohne Änderungen auch ohne die Förderung durch Take Off hätte durchgeführt werden können; keine der Forschungseinrichtungen wählte zu diesem Zeitpunkt diese Antwortoption (Prognos 2009, S. 68). Bei LuFo IV liegen die Vergleichswerte bei 3 \% (Unternehmen) und 2 \% (Forschungseinrichtungen) (Groß/Kerlen 2013, S. 15). 


\section{Technologischer Spillover}

Der technologische Spillover der Forschungsergebnisse scheint relativ hoch, und dürfte großteils auf die kooperative Struktur der Projekte zurückzuführen sein. Gut die Hälfte der Unternehmen geben an, dass die technologischen Ergebnisse ihrer Projekte in anderen Unternehmen der Luftfahrtbranche (21\%) oder sogar in anderen Branchen (32\%) genutzt werden. Zu den Anwendungsfeldern zählen unter anderem die Windenergie, Meteorologie, Baustatik und die Werkstoffproduktion.

Bei den Forschungseinrichtungen geben insgesamt drei Viertel an, dass ihre Projektergebnisse auch in anderen Einrichtungen genutzt werden. $\mathrm{Zu}$ den außerhalb der Luftfahrtbranche liegenden Anwendungsfeldern gehören hierbei die Automobilindustrie, Schiffbau, stationäre Turbomaschinen, Rohrleitungen bzw. allgemeine Fertigungstechnik.

Ein damit kongruentes Ergebnis konnte im Rahmen der Fallstudien festgehalten werden. In einem Branchenprogramm wie Take Off, das zumindest für einzelne Projekte bewusst eine gewisse Durchlässigkeit hinsichtlich benachbarter Technologiefelder und Branchen zuließ, sind Technologietransfers in unterschiedliche Richtungen zwischen den Branchen und Technologiefeldern eher die Regel als die Ausnahme, und sind sehr zu begrüßen.

\section{Grafik 26 Weitere Nutzung der technologischen Ergebnisse der Forschungsprojekte}

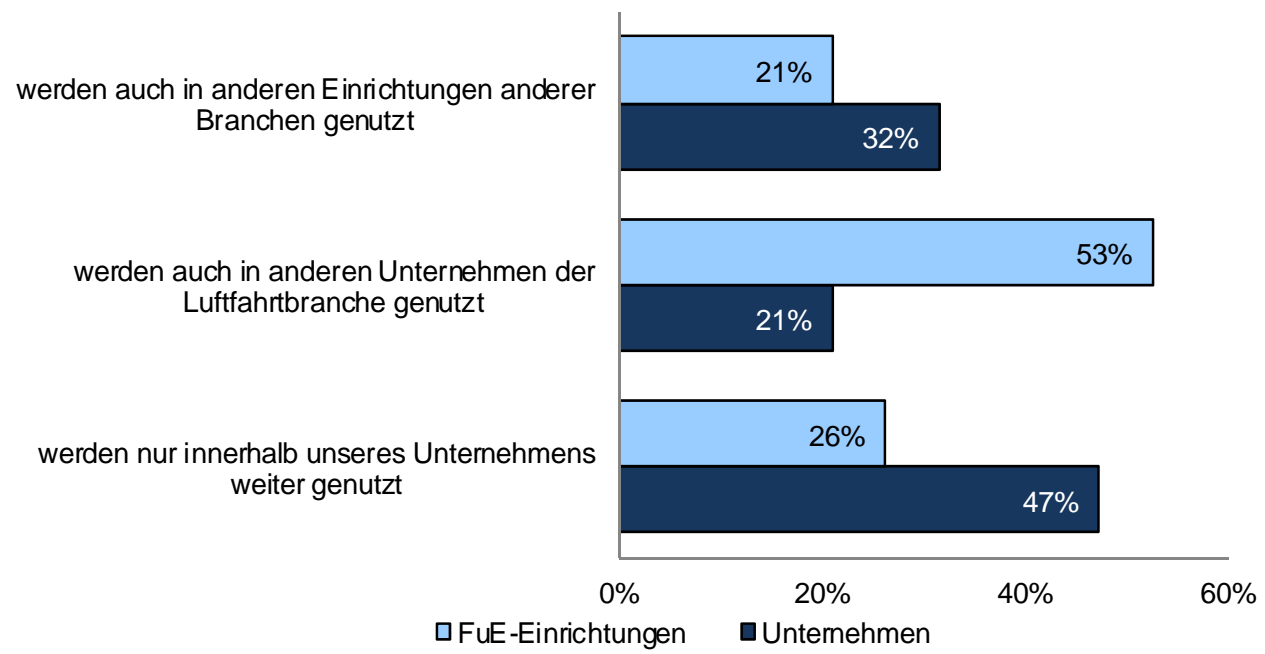

Quelle: Befragung von Unternehmen $(n=19)$ und Forschungseinheiten $(n=19)$ 


\section{Verbleib von Nachwuchskräften in der Luftfahrtbranche}

Von den Personen, denen in Unternehmen bzw. Forschungseinrichtungen im Rahmen von Take Off-geförderten Projekten die Möglichkeit eingeräumt wurde eine Dissertation anzufertigen, arbeitet die Hälfte noch in dem Unternehmen bzw. der Forschungseinrichtung, in der sie zum Zeitpunkt der Projektdurchführung tätig war. Knapp $40 \%$ sind in Unternehmen außerhalb der Luftfahrtbranche tätig. Wenn es bekannt war, wurden z.B. die Entwicklung hydraulischer Turbinen oder die stahlerzeugende Industrie genannt.

Von den Personen, die ihre Studienabschlussarbeit innerhalb von Take Off Projekten erstellten, arbeiten heute noch $54 \%$ in Unternehmen oder Forschungseinrichtungen der Luftfahrtbranche. Die übrigen sind u.a. in der Mikrosystemtechnik, der Softwareentwicklung, der Entwicklung hydraulischer Turbinen, der Prozesstechnik oder im Consulting tätig.

Jene Unternehmen, die ein Take Off Projekt durchführten und parallel auch an der Ö-LINKupdate-Studie 2009/2014 teilnahmen, konnten im Zeitraum 2009 bis 2013 ihre Beschäftigtenzahl um rd. $18 \%$ erhöhen (Spezialauswertung aus Brimatech 2015; Ö-Linkupdate-Studie $13 \%$ ). Diese Erhöhung ist überproportional in qualifizierte Stellen geflossen. Gemäß der ÖLink-Studie 2015 scheint diese Entwicklung jedoch allein von einem Großunternehmen getragen zu sein, was bereits den angesprochenen Konzentrationsprozess in der Branche nahelegt. In den Fallbeispielen dieser Evaluierung konnten jedenfalls bei Unternehmen der Branche durchaus positive Effekte dargestellt werden, die jedoch in einem komplexen Geflecht mit benachbarten Branchen (insb. Automobil) interagieren. Dieser Trend scheint derzeit nicht stark von der Konjunktur abzuhängen, sondern vielmehr von den durch die OEMs verursachten Konzentrationstendenzen. Damit wird weiterhin in der Branche ein Beschäftigungspotenzial für gut ausgebildete Absolventen gesehen.

\section{Weiterführung der Kooperation}

Eine nachhaltige Verbesserung der Kooperationsbeziehungen kann das Programm insbesondere bei den Forschungseinrichtungen bewirken (vgl. Abbildung 2). Knapp die Hälfte der Forschungseinrichtungen konnte neue, dauerhafte Kooperationsbeziehungen etablieren. Insgesamt sind die Ergebnisse zur Fortsetzung der Kooperationen als sehr positiv zu beurteilen. Dabei werden die meisten Kooperationsbeziehungen in Folgeprojekten durchgeführt (vgl. Abbildung 7). Nur jeweils $6 \%$ der Unternehmen und Forschungsinstitute planen gar keine weitere Zusammenarbeit. Das Ausschließen der weiteren Zusammenarbeit ist vorrangig auf den fehlenden Projekterfolg der jeweiligen Forschungsvorhaben und daraus resultierende, zum Teil finanzielle Einbußen zurückzuführen. 
Grafik 27 Fortsetzung der Zusammenarbeit mit den Partnern des Kooperationsprojektes nach Ende des Projektes

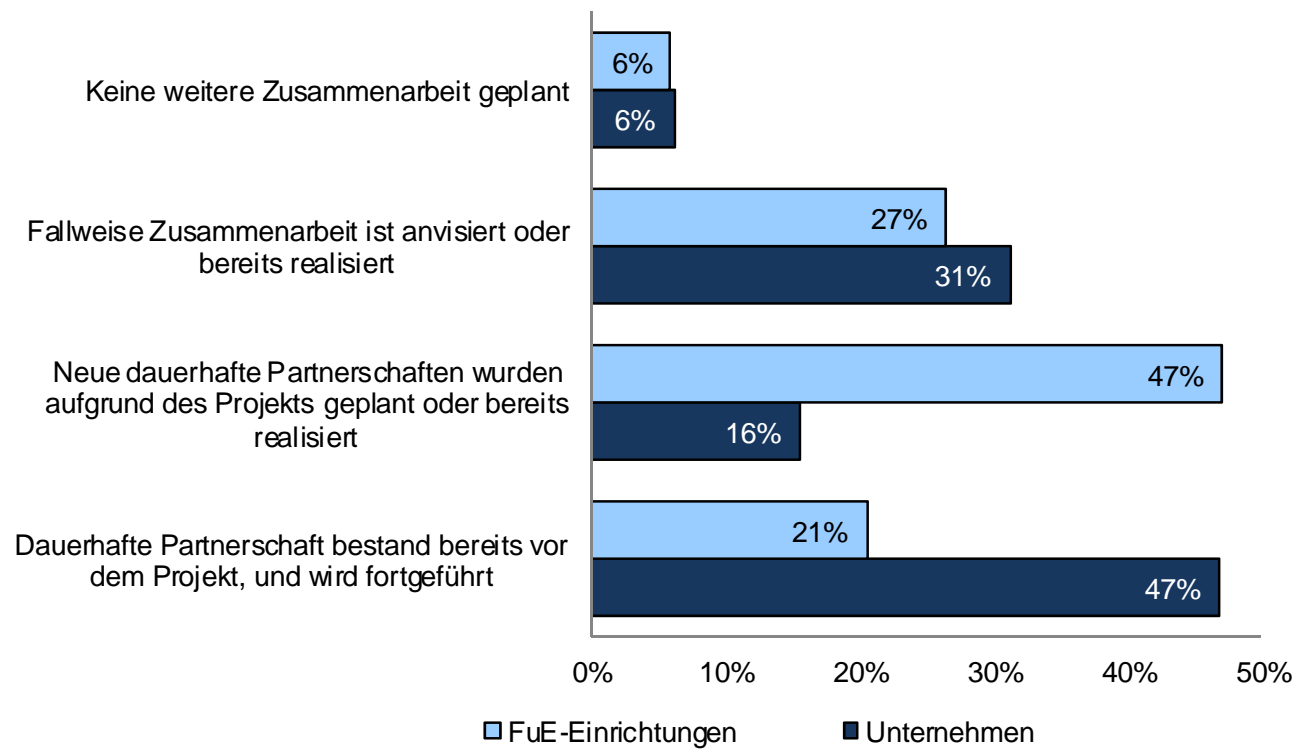

Quelle: Befragung von Unternehmen und Forschungseinheiten, $n=66$

\section{Grafik 28 Form der Fortsetzung der weiteren Zusammenarbeit}

ohne konkrete Projekte weitergeführt

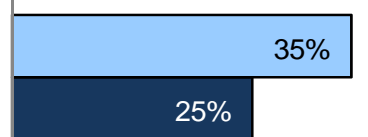

in gemeinsamen wissenschaftlichen Publikationen weitergeführt
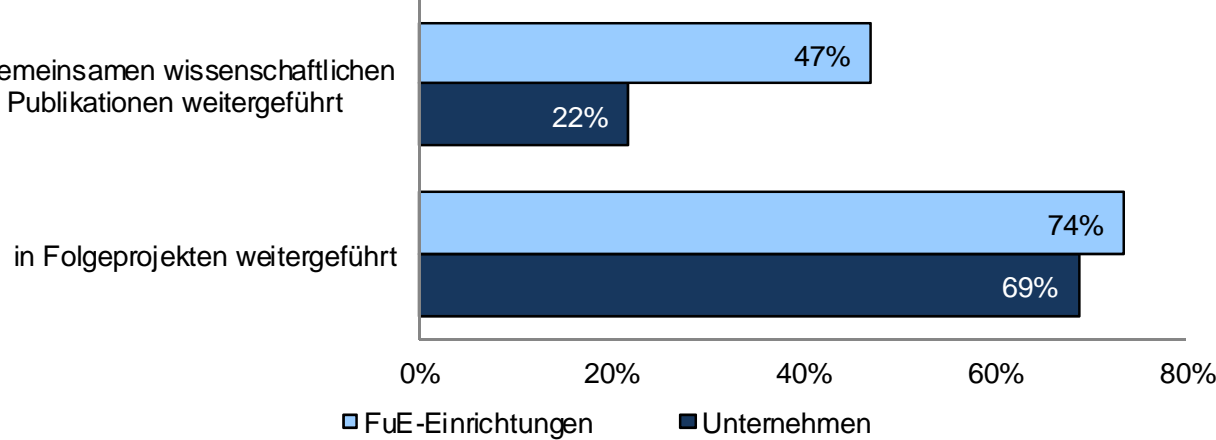

Quelle: Befragung von Unternehmen und Forschungseinheiten, $n=59$ 


\section{Grafik 29 Stärkere Integration in FuE- und Innovationskooperationen}

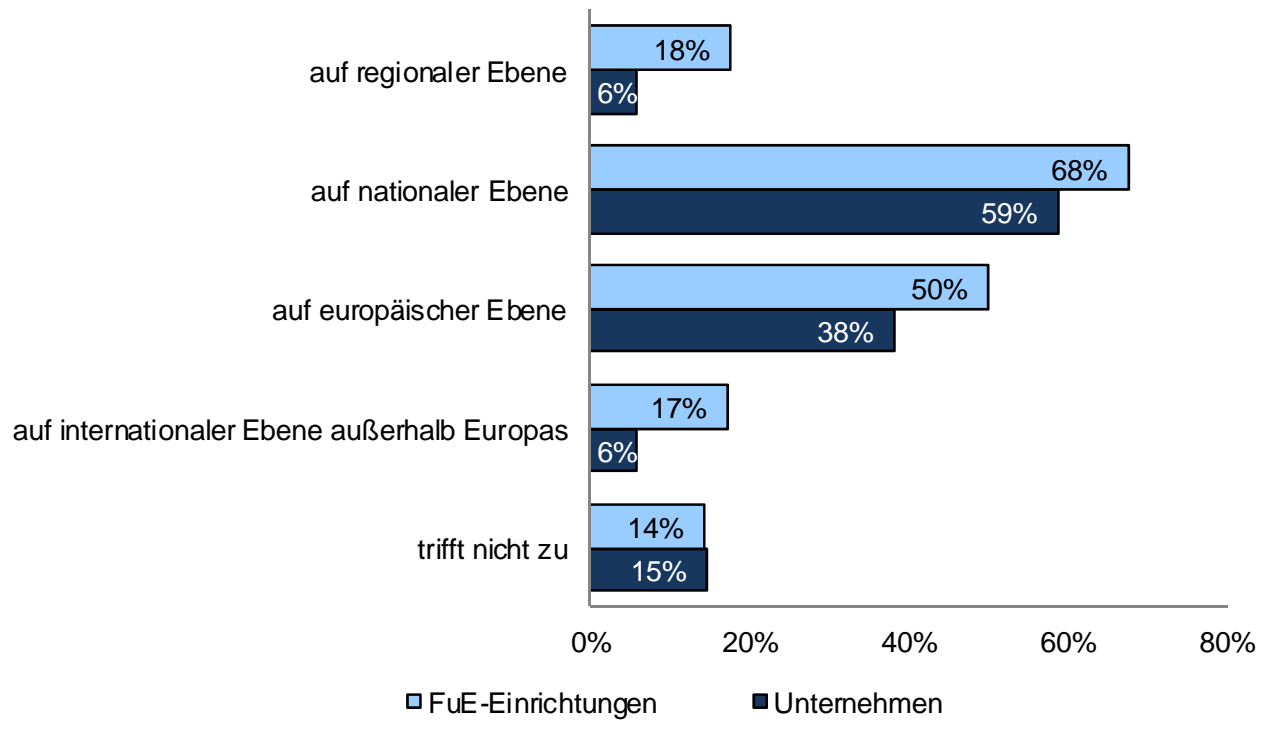

Quelle: Befragung von Unternehmen und Forschungseinheiten, $n=67$

Die Integrationswirkung des Programms bezieht sich in erster Linie auf Österreich, aber auch die Einbindung in europäische Innovationsnetzwerke wird deutlich gestärkt.

Bedeutung der Förderung für die Marktstellung der Unternehmen und die wissenschaftliche Position der Forschungseinrichtungen

Die gelingende nationale Vernetzung ist bedeutsam für die an Take Off teilnehmenden Unternehmen, weil sie ihre Marktposition mehrheitlich national definieren. Bei den österreichischen Unternehmen handelt es sich vorwiegend um KMU, die für sich nicht in Anspruch nehmen können, ein wichtiger Akteur in der internationalen Luftfahrtbranche zu sein. Die wenigen Großunternehmen sind jedoch stark mit KMU als Vorlieferanten verbunden. Die Forschungseinrichtungen sind in überwiegender Mehrheit als wichtige Akteure in die internationale Scientific Community eingebunden, was bei einer starken internationalen Vernetzung der Luftfahrtforschung auch zu erwarten ist. Ein kleinerer Anteil sieht sich darüber hinaus als Teil einer Gruppe von weltweit führenden Einrichtungen ihres Forschungsgebiets. Dieser umfasst sowohl außeruniversitäre als auch universitäre Einrichtungen. 
Grafik 30 Marktposition im internationalen Markt der Luftfahrt bzw. der Scientific Community

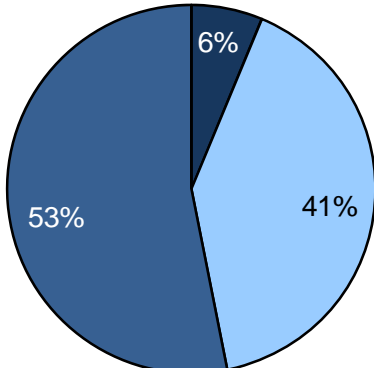

- Wir sind Marktführer

$\square$ Wir sind ein wichtiger Akteur

$\square$ Wir spielen eher im nat. Kontext eine Rolle

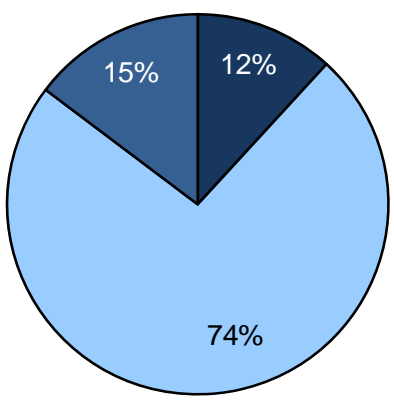

Wir gehören zu einer kleinen Gruppe von weltweit füh renden Einric 口Wir sind ein wichtiger Akteur der internationalen Scientific Commur 口Wir spielen eher im nationalen Kontext eine Rolle.

Quelle: Befragung von Unternehmen $(n=34)$ und Forschungseinheiten $(n=34)$

Der Beitrag von Take Off zur Verbesserung ihrer eigenen Position wird von den Forschungseinrichtungen höher bewertet als von den Unternehmen. Letztere geben zu knapp einem Viertel an, dass die Förderung durch Take Off gar keinen oder keinen nennenswerten Beitrag zur Verbesserung ihrer Marktposition geleistet hat. Hier finden sich auch Antworten von sehr großen Unternehmen, bei denen davon auszugehen ist, dass die Wirkung eines einzelnen Forschungsprojekts kaum der Stellung des Unternehmens zugerechnet werden kann. Da der Aufbau einer guten Marktposition in der Regel einen langen Zeitraum in Anspruch nehmen wird (lange Technologieentwicklungszeiten, starke internationale Vernetzung) ist hier positiv hervorzuheben, dass über die Hälfte der Unternehmen einen starken oder sehr starken (Werte 2 und 1 zusammen betrachtet) Einfluss von Take Off auf die Verbesserung ihrer Marktposition sieht. Bei den Forschungseinrichtungen hingegen sind zwei Drittel der Überzeugung, dass die Teilnahme an Take Off zu einer Verbesserung ihrer Position in der Scientific Community beigetragen hat. In diesem Punkt hat Take Off also in der Wissenschaft einen stärkeren Effekt erzielt als in der Wirtschaft. Es ist jedoch auch zu beachten, dass eine Veränderung der Position in der Wissenschaft schneller zu erzielen sein wird als in der Wirtschaft. 
Grafik 31 Beitrag von Take Off zur Verbesserung der eigenen Marktposition bzw. Position in der Scientific Community

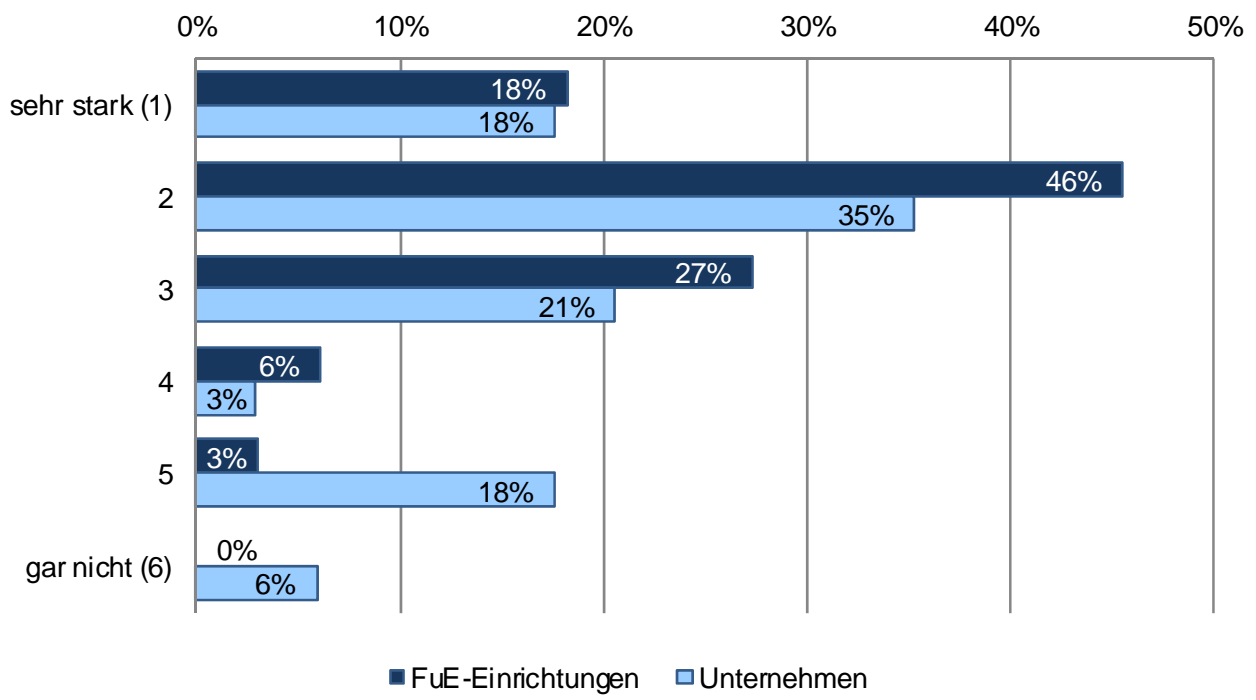

Quelle: Befragung von Unternehmen und Forschungseinheiten, $n=67$

Da für die meisten Unternehmen Luftfahrt als Geschäftsfeld von entscheidender Bedeutung ist und ihre Anstrengungen in der Luftfahrt wachsen werden, ist der Beitrag von Take Off hier als besonders relevant zu beurteilen. Dies gilt insbesondere weil Take Off das wichtigste Förderprogramm für die Unternehmen der Luftfahrtbranche ist. Darüber hinaus ist auch das FFG-Basisprogramm für Luftfahrtunternehmen ein wichtiges Förderprogramm, das allerdings überwiegend von mittleren und Großunternehmen der Luftfahrtzulieferindustrie in Anspruch genommen wird. Die meisten FFG Neukunden aus der Luftfahrtzulieferindustrie beanspruchen Take Off als Erstförderung (68\%; siehe Pseiner 2014). ${ }^{6}$ Anhand der Sonderauswertung der Ö-LINK-Studie lässt sich darüber hinaus auch erkennen, dass Unternehmen, die bisher nicht an Take Off teilgenommen haben auch relativ öfter kein substituierendes Förderprogramm in Anspruch nahmen. Mehr als die Hälfte (54\%) der relevanten Unternehmen haben keine Förderung bezogen.

${ }^{6}$ https://www.ffg.at/take-off/veranstaltung/oesterr-FTI-Luftfahrtstrategie (5.12.2014) 
Grafik 32 Inanspruchnahme weiterer Förderangebote durch Take Off teilnehmende und nicht-teilnehmende Unternehmen der Luftfahrtzulieferindustrie

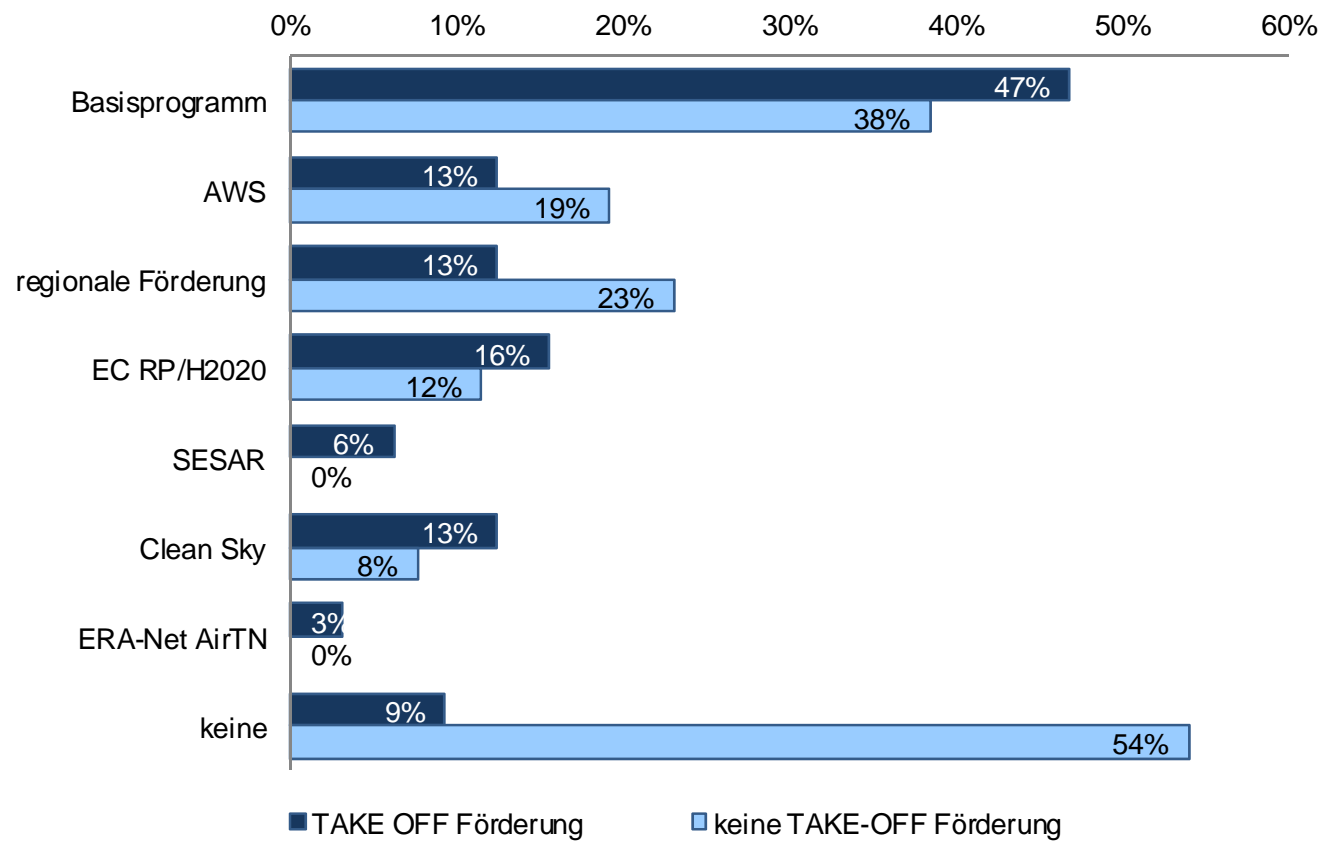

Quelle: Basiert auf den von Brimatech bereitgestellten Analyseergebnissen von anonymisierten, kumulierten Daten, die im Rahmen des Projektes Ö-LINKupdate 2015 bzw. Ö-LINK erhoben wurden und den Zeitraum 2009 bis 2013 abdecken, n=91 Unternehmen

Unternehmen mit Take Off Förderungen weisen, verglichen mit den Resultaten des Ö-LINK-Updates, dem Geschäftsfeld der Luftfahrt eine höhere Bedeutung zu als nicht-geförderte Unternehmen. Fast die Hälfte der Fördernehmer bezeichnet die Luftfahrt als entscheidendes (= primäres) Geschäftsfeld der Unternehmenstätigkeiten gegenüber $26 \%$ der Unternehmen die keine Förderung erhalten haben. Trotz dieses Unterschieds bei der Priorisierung des Geschäftsfeldes Luftfahrt strebt die Branche in ihrer Gesamtheit eine Ausweitung der Anstrengung an. Mehr als drei Viertel der befragten Unternehmen betrachten das Luftfahrtsegment als ein wachsendes Geschäftsfeld. 


\section{Grafik 33 Bedeutung von Luftfahrt als Geschäftsfeld}

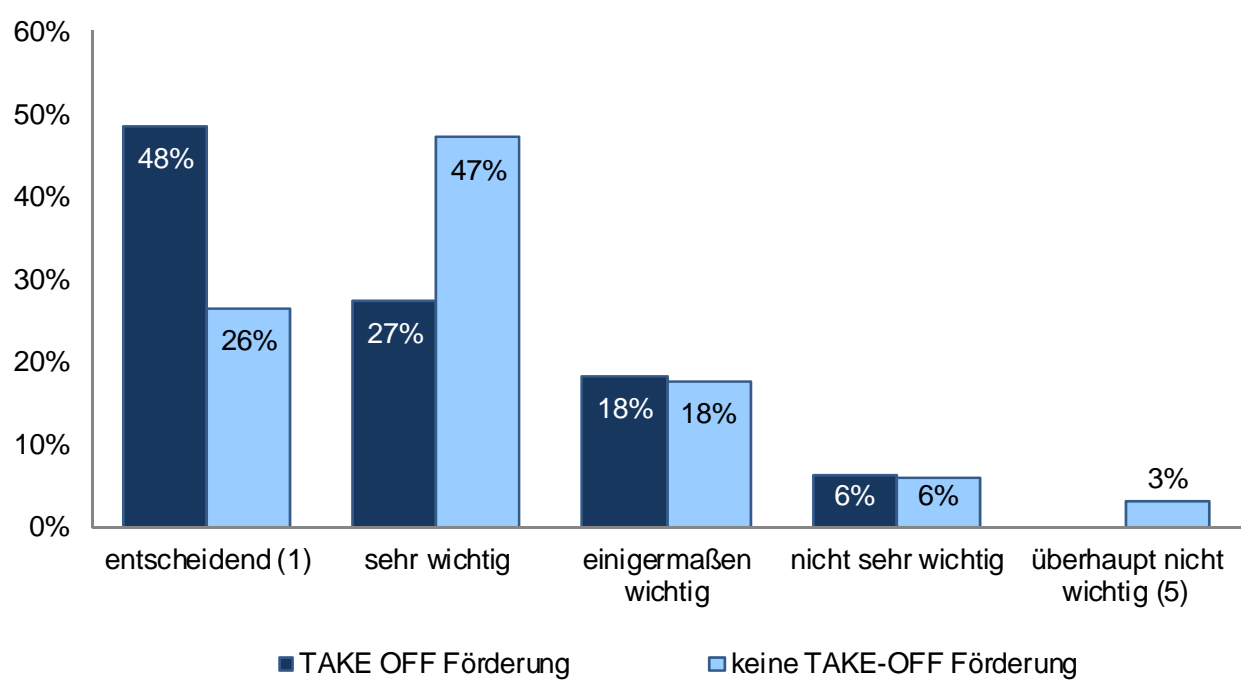

Quelle: Basiert auf den von Brimatech bereitgestellten Analyseergebnissen von anonymisierten, kumulierten Daten, die im Rahmen des Projektes Ö-LINKupdate 2014 bzw. Ö-LINK erhoben wurden; $\mathrm{n}=33$ Unternehmen

Die Unternehmen mit Take Off Förderungen, die auch an der Ö-LINK-Studie 2014 teilnahmen, konnten ihren Umsatz von 2008 auf 2013 um rd. 43 \% steigern (Spezialauswertung aufgrund von Brimatech 2015). Damit liegt das Umsatzwachstum leicht über dem Umsatzwachstum der übrigen Unternehmen in der Luftfahrtbranche, dieses liegt bei rd. $40 \%$.

\section{Wirtschaftliche Verwertung der Ergebnisse}

Über die Hälfte der Unternehmen geben an, dass die Projektergebnisse wirtschaftlich verwertet werden können. Hingegen geben lediglich $6 \%$ an, dass die Ergebnisse gar nicht im Unternehmen verwertet werden können. 


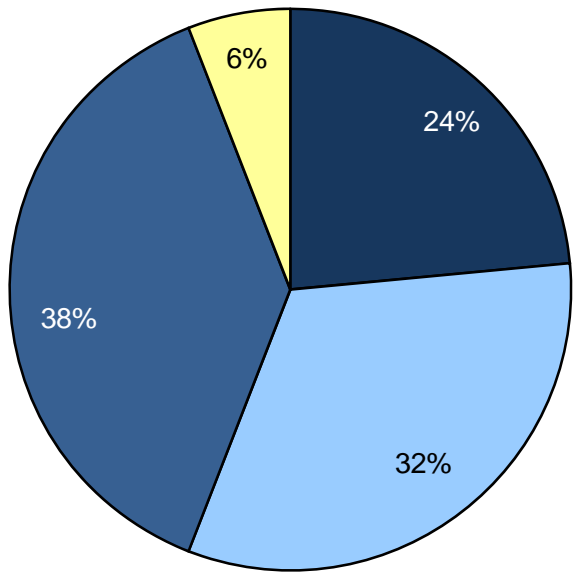

$\square$ werden in unserem Unternehmen bereits wirtschaftlich verwertet

$\square$ werden in unserem Unternehmen wirtschaftlich verwertet werden können

$\square$ werden in unserem Unternehmen nicht wirtschaftlich verwertet werden können, sind jedoch im Sinne einer Erkenntniserweiter sinnvoll

口können in unserem Unternehmen nicht verwertet werden

Quelle: Befragung von Unternehmen, $n=34$

Zum Vergleich, im FFG Wirkungsmonitoring werden regelmäßig knapp $60 \%$ der Projektergebnisse vier Jahre nach Projektende bereits wirtschaftlich verwertet, weitere rd. $10 \%$ werden in Zukunft wirtschaftlich verwertet werden können. Dies veranschaulicht den bekannten Sachverhalt, dass die Verwertungsperspektiven in der Luftfahrt um einiges langfristiger sind.

Die bereits eingetretenen wirtschaftlichen Effekte sind dabei äußerst beachtlich. Lediglich fünf Unternehmen geben an, dass sie bereits ökonomische Effekte (Zusatzumsätze, Umsatzsicherung, Kosteneinsparung, Lizenzeinnahmen, etc.) realisieren konnten. Dass dieser Wert niedrig ausfält entspricht der Grundannahme, dass nicht zuletzt aufgrund der langfristigen Entwicklungszyklen in der Luftfahrt ein hohes wirtschaftliches Risiko für die Unternehmen mit diesen Projekten verbunden ist. Diese fünf Unternehmen konnten jedoch mit 82,8 Mio. Euro in Summe einen 8,5fach höheren ökonomischen Effekt für sich realisieren, als an Förderung an alle antwortenden Unternehmen ausgeschüttet wurde. Sie prognostizieren zudem weitere positive Effekte in Höhe von 47,9 Mio. Euro bis 2020. Diese Werte sind insofern plausibel, da es sich u.a. um ein Projekt handelt, das bereits 2004 abgeschlossen wurde. 
Tabelle 7 Bereits eingetretene ökonomische Effekte (in Euro) aufgrund der Projektergebnisse

\begin{tabular}{|l|r|r|r|r|c|}
\hline & N & Minimum & Maximum & Summe & $\begin{array}{c}\text { Verhältnis zur Förder- } \\
\text { summe aller Antwor- } \\
\text { tenden }\end{array}$ \\
\hline bis 2013 & 5 & 10.000 & 80.000 .000 & 82.760 .000 & 8,5 \\
\hline 2013 bis 2020 & 5 & 100.000 & 40.000 .000 & 47.900 .000 & 4,9 \\
\hline
\end{tabular}

Quelle: Befragung von Unternehmen, $n=5$

Wesentlich vorsichtiger in ihren Prognosen sind jene Unternehmen, die noch keine ökonomischen Effekte realisiert haben. Sie gehen von einem positiven Effekt von in Summe $€ 7,2$ Mio. bis 2020 für ihre Unternehmen aus.

Tabelle 8 Voraussichtlich eintretende ökonomische Effekte (in Euro) aufgrund der Projektergebnisse

\begin{tabular}{|l|r|r|r|r|c|}
\hline & N & Minimum & Maximum & Summe & $\begin{array}{c}\text { Verhältnis zur Fördersum- } \\
\text { me aller Antwortenden }\end{array}$ \\
\hline Bis 2020 & 8 & 10.000 & 5.000 .000 & 7.260 .000 & 0,7 \\
\hline
\end{tabular}

Quelle: Befragung von Unternehmen, $\mathrm{n}=8$

Immerhin $50 \%$ der Forschungseinrichtungen streben eine wirtschaftliche Verwertung der Projektergebnisse an. Dieser Wert ist als relativ hoch zu bewerten, da die wirtschaftlich Verwertung von Projektergebnissen nicht primäres Ziel von Forschungseinrichtungen ist, passt aber mit den relativ hohen Zahl an Patentanmeldungen durch FE zusammen. 


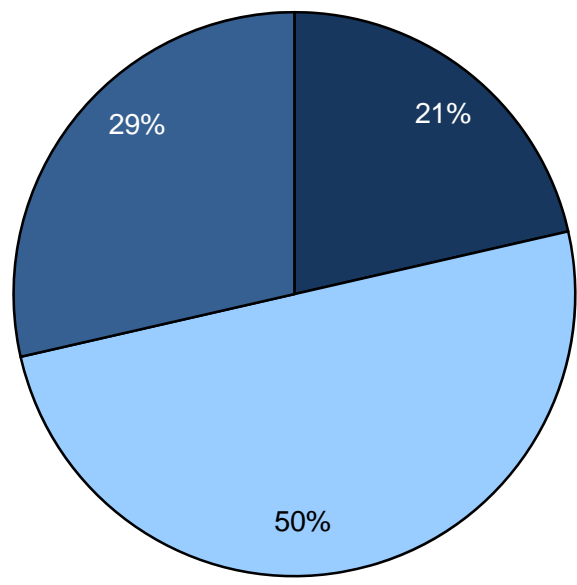

$\square$ werden in unserer Einrichtung wirtschaftlich verwertet z. B. im Sinne von zusätzlichen Drittmitteln, Lizenzeinnahmen

$\square$ werden in unserer Einrichtung wirtschaftlich verwertet werden können

$\square$ werden in unserem Unternehmen nicht wirtschaftlich verwertet werden können, sind jedoch im Sinne einer Erkenntniserweiterung sinnvoll

Quelle: Befragung von Forschungseinheiten, n=14

Drei Forschungseinrichtungen berichten von bereits eingetretenen ökonomischen Effekten. Hier handelt es sich um zusätzliche Drittmittel, Lizenzeinnahmen oder Ähnliches. Diese Werte sind erwartungsgemäß sehr viel niedriger als bei Unternehmen. Es ist eher positiv zu vermerken, dass überhaupt nennenswerte ökonomische Effekte realisiert werden können.

Tabelle 9 Bereits eingetretene ökonomische Effekte (in Euro) aufgrund der Projektergebnisse

\begin{tabular}{|l|r|r|r|r|c|}
\hline & N & \multicolumn{1}{|l|}{ Minimum } & Maximum & Summe & $\begin{array}{l}\text { Verhältnis zur Fördersumme } \\
\text { aller Antwortenden }\end{array}$ \\
\hline bis 2013 & 2 & 45.000 & 50.000 & 95.000 & 0,02 \\
\hline $\begin{array}{l}2013 \text { bis } \\
2020\end{array}$ & 3 & 100.000 & 700.000 & 950.000 & 0,2 \\
\hline
\end{tabular}

Quelle: Befragung von Unternehmen, $\mathrm{n}=5$

\section{Verbleib qualifizierter Fachkräfte in Österreich}

Es kann davon ausgegangen werden, dass die überwiegende Mehrheit der Personen, denen im Rahmen von Take Off-geförderten Projekten die Möglichkeit eingeräumt wurde, eine Dissertation oder Studienabschlussarbeit anzufertigen, nach wie vor in Österreich tätig ist. Auch wenn in der schriftlichen Befragung nicht explizit nach dem Verbleib in Österreich gefragt wurde, hat lediglich ein Antwortender im freien Antwortfeld explizit auf die Abwanderung der qualifizierten Personen nach Deutschland hingewiesen. 


\subsection{Fallbeispiele}

Die nachstehende Zusammenfassung zeigt im Überblick die wesentlichsten Resultate der geförderten Beispielprojekte, die in weiterer folge verkürzt beschrieben werden. Die detaillierten Fallstudien liegen dem BMVIT vor, können aber aufgrund des hohen Detailgehalts nicht veröffentlicht werden.

Die Ergebnisse lassen sich, durchaus in Übereinstimmung mit den Resultaten der online-Befragung von Unternehmen und Forschungseinrichtungen sowie der akademischen Literatur, wie folgt zusammenfassen:

Es ist eine höhere Inputadditionalität bei Projekten mit höheren Technology Readiness Levels (TRL) als Ausgangsbasis zu beobachten. Dies entspricht dem bekannten Phänomen, dass Unternehmen aufgrund des technologischen Risikos weniger gewillt sind eigene Mittel zu investieren, je näher ein Forschungsthema an der Grundlagenforschung liegt.

In allen Projekten ist (teilweise sehr) hohe Verhaltensadditionalität (Know-how Zuwachs aufgrund von thematischer Vertiefung, neuen Kooperationen, etc.) zu beobachten. Dies spiegelt das Faktum wider, dass die Luftfahrtindustrie und -forschung in Österreich noch relativ jung ist, und während des letzten Jahrzehnts in relevante Themengebiete neu eindrang.

Insbesondere bei den Projekten der Technologieplattform Offset ist ein hoher Vorbereitungsaufwand für einzelne Projekte zu beobachten. Damit sind jedoch teilweise völlig neue Akteurskonstallationen entstanden, die für die beteiligten Unternehmen und Forschungseinrichtungen über das einzelne Projekt hinaus hohen Nutzen für die Folgeforschung bedeuten.

In einem Branchenprogramm wie TAKE OFF, das zumindest für einzelne Projekte bewusst eine gewisse Durchlässigkeit hinsichtlich benachbarter Technologiefelder und Branchen zuließ, sind Technologietransfers in unterschiedliche Richtungen zwischen den Branchen und Technologiefeldern eher die Regel als die Ausnahme, und sind sehr zu begrüßen.

Die folgende Tabelle veranschaulicht die Input-, Verhaltens- und Outputadditionalität der untersuchten Projekte bzw. Projektketten im Überblick. 
Tabelle 10 Input-, Verhaltens- und Outputadditionalität der vier Fallstudien

\begin{tabular}{|l|c|c|c|c|c|}
\hline & Projekt A & \multicolumn{2}{|c|}{ Projekt B } & Projekt C & Projekt D \\
\hline Additionalität & Luftfahrt & Automobil & Luftfahrt & Luftfahrt & Luftfahrt \\
\hline Input & ++ & ++ & & & \\
\hline Verhalten & + & ++ & ++ & ++ & ++ \\
\hline Output & $\begin{array}{c}++ \\
\text { Zivile Lufffahrt }\end{array}$ & $\begin{array}{c}++ \\
\text { Werkstoffteile } \\
\text { und } \\
\text { Sensortechnik }\end{array}$ & & $\begin{array}{c}(+) \\
\text { Maschinenbau }\end{array}$ & $\begin{array}{c}(+) \\
\text { Bauwerküberwa } \\
\text { chung, } \\
\text { Bahnverkehr }\end{array}$ \\
\hline
\end{tabular}

Quelle: KMU Forschung Austria und Partner

\section{Projekt A}

Ziel des Projektes war die Entwicklung modularer Prüftechnologien für den zivilen Luftfahrtbereich. Durch Kombinierbarkeit wiederverwendbarer Serienkomponenten wurde die Substituierbarkeit kostenintensiver Sondermaschinen durch eine „individuelle Produktfamilie" angestrebt. Eingeordnet in die TAKE OFF Strategie trägt das Projekt zu einem sichereren und effizienten Luftransportsystem bei. Über eine Projektdauer von 2,5 Jahren arbeitete ein österreichisches Konsortium aus zwei wissenschaftlichen und unternehmerischen Partnern an der Umsetzung. Kooperationen der beteiligten Konsortialpartner sollten in weiterer Folge auch über die Projektdauer hinaus bestehen.

Die angestrebte Technologie baute auf dem Wissen bereits vorhandener Systeme der militärischen Luftfahrt auf und sollte dem projektführenden Unternehmen den technologiespezifischen Zugang zum zivilen Markt ermöglichen. Letzteres ist durch Erreichen der technischen und wirtschaftlichen Projektziele gelungen, wenngleich dafür Aufwendungen über die veranschlagten Projektkosten hinaus erforderlich waren. Das Erreichen Letzterer war ein wesentliches Projektkriterium, da das betreffende Marktsegment durch hohe Konkurrenz gekennzeichnet ist. Bereits während der Projektdurchführung waren potenzielle Kunden in die Entwicklungstätigkeiten eingebunden (da bereits Kunden des Unternehmens) wodurch es gelang, die Marktfähigkeit des angestrebten Systems während der Laufzeit zu validieren.

Es gelang mit Hilfe des modularen Prüfsystems nicht nur am zivilen Luftfahrmarkt konkurrenzfähig zu werden, sondern trugen die innovativen Komponenten auch zum Anstieg der Beschäftigten- und Umsatzzahlen bei. Eine Konkretisierung der Verwertungsergebnisse ist auf Grund der umfassenden Wachstumsphase des Unternehmens in diesem Zeitraum allerdings nicht möglich. Darüber hinaus konnte auch das eingebundene Partnerunternehmen, durch die Erfüllung der betrauten Entwicklungstätigkeiten, eine Erweiterung des Angebotsportfolios erzielen (im Bereich Messtech- 
nik). Weiters profitierten die wissenschaftlichen Partner durch Überführung der interdisziplinären FuE-Ergebnisse in die eigene Forschungstätigkeit (z.B. Kenntnisse im Bereich Konstruktion und Simulation) bzw. floss das gewonnene Know-how und die Methodenanwendung in die akademische Lehre ein.

\section{Projekt B}

Das Projekt wurde im Rahmen der der Technologieplattform Offset durchgeführt, die Projektdauer betrug drei Jahre. Unterstützt wurde das Projekt auch durch das FFG Basisprogramm. Das durchführende Konsortium bestand aus zehn Unternehmen die in Österreich und Deutschland ansässig sind.

Inhalt des Projekts war die Entwicklung und der Einsatz von Automatisierungstechnologien. Die Projektidee wurde von einem deutschen Projektteilnehmer angestoßen und resultierte aus der steigenden Nachfrage nach Kohlefaserverbundbauteilen in und abseits der Luftfahrtbranche (z.B. Automobil- und Medizintechnik). Im Rahmen von TAKE OFF nimmt das Projekt daher durchaus eine besondere Stellung ein, da es nicht nur zur nachhaltigen, internationalen Verknüpfung der beteiligten Unternehmen kam, sondern auch zu branchenübergreifenden Vernetzungen. Das eingebrachte Wissen und die Fertigkeiten der involvierten Unternehmen ermöglichte die vollständige Umsetzung des Vorhabens von der Idee bis zum Prototypen.

Der entwickelte Prototyp konnte drei Jahre nach Projektende in die Serienfertigung übergeführt werden und somit nicht nur die technischen sondern auch die wirtschaftlichen Projektziele umgesetzt werden. Neben dem Hauptprojektziel flossen erreichte Innovationen in FuE auf unternehmensindividueller Ebene ein (Materialentwicklung, Werkstoffprüfung, Verfahrenstechnologien) bzw. kam es für eine Reihe der hinzugezogenen Konsortialpartnern zur wirtschaftlichen Verwertung der entwickelten Projektbausteine (Fahrzeugbau, Sensortechnologie). Vorrangig erfolgte der Technologietransfer in Bereichen der Automobilindustrie, etwa als Grundbaustein aktueller Technologien. Auch erwiesen sich die Technologien als Türöffner in neuen Unternehmenssparten. Neben der Generierung von Zusatzumsätzen und Erschließung von Geschäftsfeldern kam es mitunter auch zur (tlw. direkt attribuierbaren) Ausweitung des Beschäftigtenstandes.

Hervorzuheben ist, dass das Netzwerk der Projektpartner so gut funktionierte, dass Folgeprojekte bald im Raum standen. Darüber hinaus wurden die erzielten Resultate mit Hilfe von Folgeprojekten explizit weiterverfolgt, um diese in der Luftfahrt zur Anwendung zu bringen.

\section{Projekt C}

Ziel des Projektes war den Stand der Technik hinsichtlich der Auslegung und Berechnung von Flugtriebwerken zu verbessern. Über eine Projektdauer von drei Jahren arbeitete ein österreichisch-deutsches Konsortium aus einem wissenschaftlichen und zwei unternehmerischen Partnern an der Umsetzung. 
Aus technischer Sicht lag das Projektziel vor allem in der Entwicklung empirischer Modelle zur Relaminiarisierung. Diese konnten im Rahmen eines EU-Projektes auch validiert werden. Daraus resultierte die Bereitstellung von „CFD Best Practice Richtlinien“ für Übergangskanäle für zukünftige Triebwerksauslegungen. Das neuentwickelte Modell für verzögerte Strömungsmodellierung konnte des Weiteren bereits in Projekten auf EU Ebene bzw. im Rahmen von LUFO mehrmals eingesetzt werden. Als direktes Nachfolgeprojekt wurden die Ergebnisse weiterentwickelt und auch auf stark beschleunigte Strömungen ausgeweitet. Abseits der Luftfahrtbranche fanden die Modellierungslösungen Eingang in das Strömungsverhalten von Wasserturbinen.

Wirkungen auf die Kooperationstätigkeiten der Konsortialpartner haben sich im begrenzten Maße ergeben, hinsichtlich der Outputadditionalität sind die Ergebnisse jedoch überschaubar, und sind derzeit noch eher im akademischen Bereich zu finden.

\section{Projekt D}

Das Projekt wurde im Rahmen der Technologieplattform Offset durchgeführt, die Projektdauer betrug sechs Jahre, wobei es aus zwei eigenständigen Projektteilen bestand. Die durchführenden Konsortien umfassten zwölf Unternehmen, die in Österreich und Deutschland ansässig sind.

Projektziel war die Entwicklung und Verifizierung eines Monitoringsystems, das die wirksame Überwachung von Faserverbundwerkstoffen ermöglicht. Die damit verknüpften FuE-Tätigkeiten erforderten umfassendes Wissen und Fertigkeiten im Bereichen der Sensorik und den zugehörigen Auswertungslogarithmen, Messmethoden sowie der Hard- und Softwareentwicklung. Auch war die umfassende Technologiekonzeptionierung bzw. Definition der Entwicklungsschritte zu Beginn des Projektes erforderlich. Weiters sollte der Verhaltensnachweis des angestrebten Systems sowohl unter Laborbedingungen als auch im realen Einsatz erfolgen.

Es konnte im Zuge des Forschungsprojektes die technische Entwicklung eines Labordemonstrators erreicht werden, der die interdisziplinäre Integration der einzelnen Projektkomponenten darstellt. Das Projekt hat die Entwicklung einer Basistechnologie im Bereich der Schadensdetektion im Luftfahrtbereich ermöglicht. Bei diesem Themenbereich handelt es sich um eine kritische Technologie mit hohem Potenzial, allerdings schreitete die ursprüngliche Akzeptanz der Technologie (und damit ein notwendiges Anforderungsbenchmarking) langsamer voran als erwartet. Zwar kann der Demonstrator in seiner Gesamtheit derzeit nicht weiterentwickelt bzw. unter realen Bedingungen getestet werden (zeitferner Einsatz der Technologie), allerdings können die unterschiedlichen Teilkomponenten von den Konsortialmitgliedern in anderen Bereichen bzw. Projekten weitergenutzt werden (z.B. nondestructive Inspection, Bauwerküberwachung). Direkte wirtschaftliche Effekte, die 
sich auf die Verwertung der entwickelten Technologie zurückführen lassen, sind auf Grund des unvollständigen Forschungsprozesses noch nicht feststellbar.

Direkte Projektwirkungen ergeben sich etwa aus dem umfassenden Wissensaufbau, den das Projekt in unterschiedlichen Disziplinen möglich gemacht hat. Laut Konsortialpartnern war die Sensibilisierung der veränderten Kundennachfrage hin zu Systemangeboten ebenfalls ein Effekt des Projektes. Einen wesentlichen Beitrag lieferte das Projekt darüber hinaus in der nachhaltigen Vernetzung der österreichischen FuE-Community (weiterführende Kooperationen), da prägende Teilnehmer der österreichischen und auch deutschen Luftfahrtbranche in den Prozess integriert wurden. Darüber hinaus wird die erfolgreiche Entwicklung des Demonstrators auch als Referenzgewinn für die beteiligten Unternehmen bewertet. 


\subsection{Gesamtwirtschaftliche Wirkung der Investitionen in For- schung und Entwicklung der Luftfahrtbranche}

\subsubsection{Die Input-Output-Methode als Analyseinstrument}

Die Input-Output-Analyse (Miller und Blair 2007) ist eine Methode der empirischen Wirtschaftsforschung, bei der die Lieferbeziehungen zwischen einzelnen Wirtschaftssektoren abgebildet und ausgewertet werden. Unter der Annahme, dass die Lieferbeziehungen zwischen den Wirtschaftssektoren in einem konstanten linearen Zusammenhang stehen, können diese im Rahmen der Input-Output-Tabelle abgebildet werden. Darin wird der Zusammenhang zwischen Wertschöpfung- und Endnachfragekomponenten in hoher sektoraler Auflösung dargestellt. Für Österreich werden die entsprechenden Daten durch Statistik Austria bereitgestellt. Die InputOutput-Tabellen werden aus den erhoben Aufkommens- und Verwendungstabellen geschätzt. Dabei werden die Verflechtungen zwischen 74 Sektoren insgesamt und auch getrennt nach heimischen und importierten Güter dargestellt. Die letzte veröffentliche Tabelle bezieht sich auf das Jahr 2010 (Statistik Austria 2014).

Die Input-Output-Analyse bietet ein aussagekräftiges Instrument für die Abschätzung gesamtwirtschaftlicher Auswirkungen von Änderungen der Endnachfrage in einem oder mehreren Wirtschaftszweigen. In der konventionellen Schreibweise beschreibt $x$ den Output-Vektor (Produktionswert) und $f$ den Vektor der Endnachfrage. Die Matrix $A$ gibt die Lieferbeziehungen zwischen den Sektoren wieder. Dabei entsprechen die Elemente der Matrix den Input-Koeffizienten $a_{i j}$. Diese beschreiben den Anteil der Zwischenprodukte aus Sektor $i$ am Produktionswert des Sektor $j$. Der Zusammenhang zwischen Produktionswert und Endnachfrage entspricht: $x=A x+f$. Eine Änderung der Endnachfrage löst direkt Veränderungen von Produktion in mindestens einem Sektor aus. Damit hier die Produktion ausgeweitet werden kann, müssen Vorprodukte aus anderen Sektoren bezogen werden, für die wiederum Vorprodukte notwendig sind. In der Summe ergeben sich die folgenden Veränderungen:

$$
x=\underbrace{f+A f}_{\text {direkt }}+\underbrace{A^{2} f+A^{3} f+A^{4} f+\ldots}_{\text {indirekt }}
$$

Dabei bezeichnet der direkte Effekt (unmittelbar bei den Beteiligungen anfallende Wertschöpfung), den Initialeffekt aus der Endnachfrageänderung ( $f$ ) und dem Erstrundeneffekt $(A f)$. Der Erstrundeneffekt beschreibt die zusätzliche Produktion in den Zulieferbetrieben der von der Endnachfrageänderung betroffenen Wirtschaftszweige. Oftmals wird dieser Effekt bereits zu den indirekten Effekten gezählt. Die indirekten Effekte werden entlang der gesamten Wertschöpfungskette beobachtet, 
da jeder Wirtschaftszweig eine gegebene Menge an Vorleistungen für die Produktion von anderen Wirtschaftszweigen benötigt.

Durch Auflösen der Gleichung $x=A x+f$ ergibt sich die Beziehung zwischen Produktionswerten und Endnachfrage als:

$$
x=(I-A)^{-1} f
$$

Dabei ergeben sich aus der Leontief-Inversen $(I-A)^{-1}$ die Multiplikatoren, die eine Abschätzung der durch eine Veränderung der Endnachfrage ausgelösten gesamtwirtschaftlichen Produktionseffekte ermöglichen. Daraus werden dann unter der Annahme von konstanten Relationen die Bruttowertschöpfungs- und Beschäftigungsänderungen berechnet.

Es wird dann weiter angenommen, dass ein Beschäftigungsanstieg mit einem Anstieg der Einkommen verbunden ist. Die Erhöhung der Einkommen induziert dann zusätzliche Konsumausgaben und somit eine weitere Erhöhung der Endnachfrage, die wieder zu Produktionssteigerungen führt. Somit wird eine weitere Wirkungskette ausgelöst, deren Gesamteffekt als induzierter Effekt bezeichnet wird.

\subsubsection{Gesamtwirtschaftliche Effekte der Forschungs- und Entwick- lungsausgaben}

Zur Abschätzung der gesamtwirtschaftlichen Effekte der Forschungsförderung im Luft- und Raumfahrzeugbau wird zunächst ermittelt, auf welche Komponenten der Endnachfrage sich die Forschungs- und Entwicklungsausgaben auswirken. Die von Unternehmen und Forschungseinrichtungen beantragten Fördermittel stellen eine Erhöhung der Ausgaben dar und lösen so Produktions- und Beschäftigungseffekte aus. Dabei wird zwischen verschiedenen Ausgabenkategorien unterschieden. Die von der FFG zur Verfügung gestellten Daten unterscheiden zwischen Einrichtungskosten, sonstigen Kosten und Personalkosten. Im Folgenden werden die Personalkosten und die sonstigen Kosten dem Sektor „Forschung und Entwicklung“ zugerechnet. Die zur Realisierung des Forschungsvorhabens gekauften Anlagegüter wie Maschinen und Geräte werden dem Sektor „sonstiger Fahrzeugbau“, der auch den Bau von Luftfahrzeugen enthält, zugerechnet.

Die Ausgaben für Forschung und Entwicklung belaufen sich für die gesamte Förderperiode (2002-2013) auf insgesamt 119,6 Mio. Euro (58,9 Mio. Euro Förderung + 60,7 Mio. Euro Eigenanteil der Unternehmen und Forschungseinrichtungen). Der mit 104,0 Mio. größte Teil dieser Ausgaben floss direkt in den Sektor Forschung und 
Entwicklung und etwa 7,3 Mio. Euro erhöhten die Endnachfrageerhöhung im Sektor „Sonstiger Fahrzeugbau“"

Über die Gesamtsumme der Projekte hinaus wurden in den Unternehmen und Forschungseinrichtungen zusätzliche finanzielle Mittel bereitgestellt. Diese könnten zum Beispiel für die Anschaffung zusätzlicher Geräte, Lizenzen oder auch die Einstellung zusätzlichen Personals verwendet werden. Die Summe dieser Mittel von den an der Befragung teilnehmenden Unternehmen und Forschungseinrichtungen beträgt 3,2 Mio. Euro. Die Summe der Gesamtkosten der an der Befragung teilnehmenden Unternehmen und Forschungseinrichtungen liegt bei 29,2 Mio. Euro. Somit wurde durch die Befragung knapp $24 \%$ der gesamten Projektkosten abgedeckt. Im Folgenden wird angenommen, dass die Relation zwischen den Zusatzinvestitionen und Fördergeldern bei den nicht an der Befragung teilnehmenden Unternehmen Forschungseinrichtungen und den teilnehmenden identisch ist. Unter dieser Annahme können die 3,2 Mio. Zusatzinvestitionen auf 13,1 Mio. für die Gesamtheit der Fördernehmer hochgerechnet werden (Tabelle 11).

\section{Tabelle 11 Investitionen im Luftfahrzeugbau}

\begin{tabular}{|l|c|}
\hline Investitionen & Ausgaben \\
\hline & Euro \\
\hline Förderung + Eigenanteil & 119.583 .100 \\
\hline Davon & \\
\hline Ausgaben für Anlagen & 7.293 .806 \\
\hline Ausgaben für FuE & 104.031 .764 \\
\hline zusätzliche Investitionen & 13.100 .000 \\
\hline Insgesamt & $\mathbf{1 3 2 . 6 9 5 . 0 3 8}$ \\
\hline
\end{tabular}

Quellen: Take Off Unternehmensbefragung; eigene Berechnungen

Für die Berechnungen der direkten, indirekten und induzierten Effekte wurde die Input-Output-Tabelle für Österreich aus dem Jahr 2010 zu Grunde gelegt (Statistik Austria 2014). Den Berechnungen liegt die Annahme zu Grunde, dass die InputKoeffizienten über den Beobachtungszeitraum konstant sind. Dies ermöglicht, die Investitionsausgaben, die sich über mehrere Jahre erstrecken, auf Grundlage der Input-Output-Tabelle für das Basisjahr zu betrachten. Die im Folgenden angegebenen Werte beziehen sich auf die Effekte über die gesamte Förderperiode zwischen 2002 und 2013.

7 Der Luftfahrzeugbau ist Teil des Sektors „Sonstiger Fahrzeugbau“. Für die Berechnungen wird daher angenommen, dass die strukturellen Zusammenhänge des Luftfahrzeugbaus nicht signifikant von denen des "Sonstigen Fahrzeugbaus“ abweichen. 
Tabelle 12 zeigt die Abschätzung der durch die Forschungsförderung ausgelösten gesamtwirtschaftlichen Effekte. In Folge der Forschungsförderung steigt die Endnachfrage nach FuE im Inland um 117 Mio. Euro. Diese FuE-Ausgaben werden im Wesentlichen für Personal verwendet und erhöhen deshalb die Nachfrage im Inland. Außerdem steigt der Bedarf an Maschinen und Anlagen um 7,2 Mio. Aufgrund der für kleinere Länder üblichen hohen Importquote wird dieser Bedarf zu etwas mehr als $50 \%$ durch Importe gedeckt. Hier steigt die inländische Nachfrage nur um 3,6 Mio. Euro. Insgesamt steigt der Produktionswert ${ }^{8}$ in Österreich um 129 Mio. Euro. Damit steigt die Bruttowertschöpfung um 61,9 Mio. Euro und die Beschäftigung um 1.240 Personen. Der Multiplikator bezogen auf die Summe der staatlichen Förderung (ohne Eigenanteil und zusätzliche Investitionen der Unternehmen) liegt bei 5,1. Eine Forschungsförderung in Höhe von 1 Mio. Euro bewirkt somit eine zusätzliche Produktion von 5,1 Mio. Euro. Die Multiplikatoren von der direkten staatlichen Förderung zum Gesamteffekt liegen für die Bruttowertschöpfung bei 5,3 und für die Beschäftigung bei 4,2. Das bedeutet, die durch die staatliche Förderung ausgelöste zusätzliche Bruttowertschöpfung wird aufgrund der dadurch ausgelösten direkten, indirekten und induzierten Effekte entlang der Wertschöpfungskette mehr als verfünffacht.

Der Initialeffekt entspricht der durch das Förderprogramm ausgelösten Endnachfrageänderung von insgesamt 129 Mio. Euro überwiegend im Sektor „Forschung und Entwicklung" und zu einem geringen Anteil im Sektor "Sonstiger Fahrzeugbau“. Damit verbunden ist eine zusätzliche Nachfrage nach Arbeitskräften in Höhe von 1.240 Personen. Gesamtwirtschaftlich steigt die Produktion weiterhin durch die Effekte, die sich aus der gestiegenen Nachfrage nach Vorleistungsgütern und Dienstleistungen ergeben. In den Zulieferbetrieben des Sonstigen Fahrzeugbaus sowie der Forschung und Entwicklung kommt es zunächst zu einem Anstieg der Produktion um 56 Mio. Euro (Erstrundeneffekt). Dadurch werden weitere indirekte Effekte ausgelöst. Der Effekt über die gesamte Wertschöpfungskette liegt bei 214 Mio. Euro. Die zusätzliche Produktion schafft noch einmal rund 240 zusätzliche Arbeitsplätze. Dies wirkt sich darüber hinaus auf die Einkommen in Österreich aus. Die Einkommenssteigerungen induzieren weitere Wertschöpfungs- und Beschäftigungseffekte in Höhe von 37 Mio. Euro und etwa 500 Beschäftigten.

${ }^{8}$ Der Produktionswert gibt die Summe der in einer Volkswirtschaft produzierten Güter und Dienstleistungen an. Im Gegensatz zum Umsatz werden beim Produktionswert sonstige betriebliche Erträge und Bestandsänderungen berücksichtigt. Die Bruttowertschöpfung ergibt sich durch Abzug der Vorleistungen vom Produktionswert. Sie beschreibt damit den im Produktionsprozess geschaffenen Mehrwert. 
Tabelle 12 Direkte, indirekte und induzierte Effekte der FuE-Ausgaben

\begin{tabular}{|c|c|c|c|c|}
\hline & $\begin{array}{c}\text { Aufkommen } \\
\text { nach Gütern } \\
\text { (\& DL) }\end{array}$ & $\begin{array}{l}\text { Produk- } \\
\text { tionswert }\end{array}$ & $\begin{array}{l}\text { Bruttowert- } \\
\text { schöpfung }\end{array}$ & Beschäftigung \\
\hline & Mio. Euro & Mio. Euro & Mio. Euro & Anzahl \\
\hline direkt insgesamt & 208,0 & 185,4 & 85,7 & 1.581 \\
\hline \multicolumn{5}{|l|}{ davon } \\
\hline Initialeffekt insgesamt & 132,7 & 129,0 & 61,9 & 1.240 \\
\hline \multicolumn{5}{|l|}{ davon ausgelöst durch } \\
\hline staatliche Förderung & 58,9 & 57,2 & 27,5 & 550 \\
\hline Eigenanteil Unternehmen & 60,7 & 59,0 & 28,3 & 567 \\
\hline zusätzliche Investitionen & 13,1 & 12,7 & 6,1 & 123 \\
\hline Erstrundeneffekt insgesamt & 75,3 & 56,4 & 23,8 & 341 \\
\hline \multicolumn{5}{|l|}{ davon ausgelöst durch } \\
\hline staatliche Förderung & 33,4 & 25,0 & 10,6 & 151 \\
\hline Eigenanteil Unternehmen & 34,4 & 25,8 & 10,9 & 156 \\
\hline zusätzliche Investitionen & 7,4 & 5,6 & 2,3 & 34 \\
\hline indirekt insgesamt & 59,4 & 29,4 & 22,6 & 240 \\
\hline \multicolumn{5}{|l|}{ davon ausgelöst durch } \\
\hline staatliche Förderung & 26,4 & 13,1 & 10,0 & 106 \\
\hline Eigenanteil Unternehmen & 27,2 & 13,5 & 10,3 & 110 \\
\hline zusätzliche Investitionen & 5,9 & 2,9 & 2,2 & 24 \\
\hline direkt und indirekt & 267,4 & 214,8 & 108,3 & 1.821 \\
\hline \multicolumn{5}{|l|}{ davon ausgelöst durch } \\
\hline staatliche Förderung & 118,6 & 95,3 & 48,1 & 808 \\
\hline Eigenanteil Unternehmen & 122,3 & 98,3 & 49,6 & 833 \\
\hline zusätzliche Investitionen & 26,4 & 21,2 & 10,7 & 180 \\
\hline induziert insgesamt & 97,9 & 78,3 & 37,0 & 503 \\
\hline \multicolumn{5}{|l|}{ davon ausgelöst durch } \\
\hline staatliche Förderung & 43,4 & 34,7 & 16,4 & 223 \\
\hline Eigenanteil Unternehmen & 44,8 & 35,8 & 16,9 & 230 \\
\hline zusätzliche Investitionen & 9,7 & 7,7 & 3,7 & 50 \\
\hline Summe insgesamt & 365,3 & 293,1 & 145,3 & 2.324 \\
\hline
\end{tabular}

Quelle: Eigene Berechnungen 


\subsubsection{Gesamtwirtschaftliche Effekte einer Produktneueinführung}

Ein Ziel der Forschungs- und Entwicklungsförderung ist es, marktfähige Produkte hervorzubringen. Im Folgenden wird daher der gesamtwirtschaftliche Effekt einer hypothetischen Produktneueinführung dargestellt. Es wird angenommen, dass ein neues Produkt eine zusätzliche Endnachfrage von $1 \mathrm{Mrd}$. Euro erzeugt. Da für die Produktion des Gutes zahlreiche Vorleistungen notwendig sind, werden auch die Branchen von der Produkteinführung profitieren, die direkt oder indirekt entlang der Wertschöpfungskette mit dem Luftfahrzeugbau verbunden sind.

Tabelle 13 zeigt die geschätzten direkten, indirekten und induzierten Wertschöpfungs- und Beschäftigungseffekte. Um die zusätzliche Endnachfrage von $1 \mathrm{Mrd}$. Euro zu bedienen, steigt der Produktionswert in Österreich insgesamt um 1,85 Mrd. Euro. Verbunden ist dies mit einem Anstieg der Bruttowertschöpfung um 686 Mio. Euro und einem zusätzlichen Arbeitskräftebedarf von rund 7.378 Personen. Besonders stark mit dem sonstigen Fahrzeugbau verbunden sind insbesondere andere Industriebranchen, wie die Energieerzeugung, die Chemiebranche und die Metall erzeugenden und weiterverarbeitenden Industrien. Aber auch die unternehmensnahen Dienstleistungen gehören neben der Luftfahrzeugbranche selbst zu den größten Profiteuren der Produktneueinführung.

Tabelle 13 Direkte, indirekte und induzierte Effekte einer Produktneueinführung

\begin{tabular}{|l|c|c|c|}
\hline Effekte & Produktionswert & Bruttowertschöpfung & Beschäftigte \\
\hline & Mio. Euro & Mio. Euro & Anzahl \\
\hline Direkt & & & \\
\hline Initialeffekt & $1.000,0$ & 309,9 & $2.955,0$ \\
\hline Erstrundeneffekt & 446,0 & 211,0 & $2.184,7$ \\
\hline Indirekt & 116,9 & 36,7 & 490,3 \\
\hline Summe & $\mathbf{1 . 5 6 2 , 9}$ & $\mathbf{5 5 7 , 5}$ & $\mathbf{5 . 6 3 0 , 0}$ \\
\hline Induziert & 272,2 & 128,7 & $1.748,0$ \\
\hline Summe insgesamt & $\mathbf{1 . 8 3 5 , 0}$ & $\mathbf{6 8 6 , 3}$ & $\mathbf{7 . 3 7 8 , 0}$ \\
\hline
\end{tabular}

Quelle: Eigene Berechnungen 


\subsubsection{Ein Vergleich zu den Effekten der deutschen Luftfahrtforschung}

Die Effekte der Produkteinführung können direkt mit denen verglichen werden, die für Deutschland bei der Evaluation der Luftfahrtforschung in den Förderprogrammen Lufo III, IV geschätzt wurden. Ein Vergleich zwischen den Tabelle 14 und Tabelle 15 zeigt, dass die Erstrundeneffekte beim Produktionswert in Österreich und Deutschland auf einem sehr ähnlichen Niveau liegen. Die indirekten und induzierten Effekte auf den Produktionswert sind in Österreich deutlich kleiner. Der Grund liegt in höheren Importquoten, die zu kleineren inländischen Multiplikatoren führen. Deshalb sind die Effekte auf Bruttowertschöpfung und Beschäftigung in Österreich etwas kleiner als in Deutschland.

Tabelle 14 Direkte, indirekte und induzierte Effekte einer Produktneueinführung in Deutschland

\begin{tabular}{|l|c|c|c|}
\hline Effekte & Produktionswert & Bruttowertschöpfung & Beschäftigung \\
\hline & Mio. Euro & Mio. Euro & Anzahl \\
\hline Direkt & & & \\
\hline Initialeffekt & $1.000,0$ & 268,1 & 3.675 \\
\hline Erstrundeneffekt & 462,4 & 189,4 & 2.419 \\
\hline Indirekt & 353,1 & 152,9 & 1.747 \\
\hline Summe & $\mathbf{1 . 8 1 5 , 5}$ & $\mathbf{6 1 0 , 4}$ & $\mathbf{7 . 8 4 1}$ \\
\hline Induziert & 465,5 & 185,4 & 2.339 \\
\hline Summe insgesamt & $\mathbf{2 . 2 8 1 , 0}$ & $\mathbf{7 9 5 , 8}$ & $\mathbf{1 0 . 1 8 0}$ \\
\hline
\end{tabular}

Quelle: Groß, Kerlen et al. (2013) 
Vergleicht man die Multiplikatoren, die sich aus den direkten FuE-Ausgaben ergeben, sind hier die direkten Effekte in Österreich etwas günstiger. Dies liegt zum einen daran, dass in Österreich mehr zusätzliche Investitionen ausgelöst werden: In Österreich wurden Zusatzinvestitionen in etwa $10 \%$ der Fördersumme ausgelöst, wohingegen dieser Anteil in Deutschland nur bei $5 \%$ lag. Zum anderen sind die direkten Effekte in Österreich etwas günstiger, da hier ein etwas höherer Anteil der Fördergelder in den Sektor „Forschung und Entwicklung“ floss und dort vollständig nachfragewirksam wurde. Die günstigeren Multiplikatoren im Initialeffekt gleichen die etwas schlechteren Multiplikatoren in den späteren Stufen aus, so dass sich insgesamt - trotz der höheren Importe in Österreich - Multiplikatoren in ähnlicher Größenordnung wie in Deutschland ergeben.

Tabelle 15 Multiplikatoren der FuE-Ausgaben in Österreich und in Deutschland

\begin{tabular}{|c|c|c|c|c|c|c|}
\hline & \multicolumn{3}{|c|}{ Österreich } & \multicolumn{3}{c|}{ Deutschland } \\
\hline & $\begin{array}{c}\text { Produk- } \\
\text { tionswert }\end{array}$ & $\begin{array}{c}\text { Brutto- } \\
\text { wert- } \\
\text { schöp- } \\
\text { fung }\end{array}$ & $\begin{array}{c}\text { Beschäf- } \\
\text { tigung }\end{array}$ & $\begin{array}{c}\text { Produk- } \\
\text { tionswert }\end{array}$ & $\begin{array}{c}\text { Brutto- } \\
\text { wert- } \\
\text { schöp- } \\
\text { fung }\end{array}$ & $\begin{array}{c}\text { Beschäf- } \\
\text { tigung }\end{array}$ \\
\hline Initialeffekt & 2,3 & 2,3 & 2,3 & 2,1 & 2,1 & 2,1 \\
\hline Direkt insgesamt & 3,2 & 3,1 & 2,9 & 3,0 & 3,4 & 3,1 \\
\hline Indirekt & 0,5 & 0,8 & 0,4 & 0,5 & 0,6 & 0,5 \\
\hline Direkte und indirekt & 3,8 & 3,9 & 3,3 & 3,5 & 4,1 & 3,5 \\
\hline Induziert & 1,4 & 1,3 & 0,9 & 1,2 & 1,6 & 1,3 \\
\hline Summe insgesamt & $\mathbf{5 , 1}$ & $\mathbf{5 , 3}$ & $\mathbf{4 , 2}$ & $\mathbf{4 , 8}$ & $\mathbf{5 , 7}$ & $\mathbf{4 , 9}$ \\
\hline
\end{tabular}

Quelle: Groß, Kerlen et al. (2013); eigene Berechnungen

Betrachtet man die beschriebenen Effekte dieser I/O-Analyse im Kontext der allgemeinen Umsatz- und Beschäftigtenentwicklung der österreichischen, luftfahrtorientierten Unternehmen, so spiegeln sich die obigen Ergebnisse auch in den betrieblichen Kennzahlen wider. Die von der Austrian Aeronautics Industries Group angeführten Wachstumsverläufe der Luftfahrtunternehmen und Zulieferer zeigen einen deutlichen Anstieg des Umsatzes und der Beschäftigung, die mitunter auf die erwähnten Effekte zurückzuführen sind. Gemäß dem ÖLinkUpdate 2015 scheint die positive Entwicklung in den letzten Jahren (2009-2013) primär auf ein Großunternehmen zurückzuführen zu sein. 


\section{Schlussfolgerungen und Empfehlungen}

Nach den in der Evaluationsliteratur geläufigen Kriterien für die Beurteilung von Förderprogrammen werden die Ergebnisse dieser Evaluierung an dieser Stelle zusammengefasst. Darauf aufbauend werden die Handlungsempfehlungen zur weiteren Erhöhung der Effektivität und Effizienz der Programmumsetzung formuliert. Die Beantwortung der Evaluierungsfragen gemäß den Terms of Reference erfolgt sowohl in der Zusammenfassung als auch in den Handlungsempfehlungen.

\section{Relevanz und Kohärenz}

Das Strategieprogramm Take Off wurde im Jahre 2002 ins Leben gerufen, da (1) sich mittelfristig einige technologische Umbrüche in der Luftfahrt und technologisch verwandten Branchen abzeichneten (Energie- und Ressourceneinsparung in der Luftfahrt sowie E-Mobilität in der Automobilindustrie), (2) sich in Österreich eine kritische Masse an Unternehmen gebildet hatte, die steigende Umsätze in der Luftfahrtbranche erwirtschafteten, sodass eine Unterstützung aus strukturpolitischen Gründen überlegt wurde, und (3) waren diese Unternehmen in Österreich noch kaum vernetzt, und liefen aufgrund dessen Gefahr ihr volles Potential in der auf Systemintegration / Konzentration ausgerichteten Luftfahrtindustrie, mit entsprechenden Konsequenzen für die Zulieferindustrie, nicht ausschöpfen zu können. Dieser wissensintensive Markt, gekennzeichnet durch Langfristigkeit, Dominanz von wenigen multinationalen Konzernen, sowie die Integration von unterschiedlichen, vertikalen sowie Querschnittstechnologien für die Unternehmen birgt mitunter auch relativ hohe Entwicklungsrisiken für die Unternehmen (Clement und Billovits 2007, Forstinger et al. 2014).

Dieser Argumentationsstrang begründete auch die Erarbeitung der FTILuftfahrtstrategie des Jahres 2008, die fortan den formalen Rahmen für Take Off darstellte. Abgesehen von den realwirtschaftlichen Gegebenheiten leitet sich die Relevanz des Programms somit von der FTI-Luftfahrtstrategie des bmvit ab, die wiederum in die Europäische Luftfahrtforschungsstrategie eingebettet ist.

Die Kohärenz der Programmziele zueinander ist grundsätzlich gegeben; sie könnten jedoch noch präzisiert werden. Damit ist angesprochen, dass „effizient, sicher, klimaschutz- und komfortorientiert" zwar insgesamt sinnvolle Teilziele darstellen, jedoch nicht notwendigerweise zusammengehören und deshalb auch nicht zusammen diskutiert, und damit möglicherweise abgewertet werden sollten. Hier ist auf die separate Behandlung der Themen auf der Ebene der Outcomes und Impacts zu achten. Ob das Thema Komfort, das nur wenige Unternehmen in Österreich vordringlich anspricht, eine separate Ausweisung als Zielgröße rechtfertigt, sei ebenso zur Diskussion gestellt. Weiters könnten alle Ziele durch eine weitere Konkretisierung gewinnen, d.h. qualitativ oder quantitativ präzisiert werden. Zum Beispiel sind als über- 
geordnete Vorgaben für Effizienz, die Klimaschutz- sowie Sicherheitsziele die Festlegungen von Flightpath 2050 und ACARE zu nennen.

Weiters ist die Zielsetzung der Ausbildung dahingehend etwas kritisch zu hinterfragen, als mit Take Off anfangs die Weiterbildung von ForscherInnen und TechnikerInnen betrieben wurde (später dann nicht mehr ausgeschrieben), da die hauptsächlichen Effekte bezüglich Kompetenzaufbau eher in den Forschungsprojekten selbst sowie bei der Ausbildung von Nachwuchskräften in Form der Unterstützung von projektbegleitenden Dissertationen etc. zu suchen ist. Während umfassende Maßnahmen zur Ausrichtung der Ausbildung auf die Bedarfe der Industrie sicherlich begrüßenswert ist (siehe auch Drechsler et al. 2011), stellt sich die Frage, ob dies ein Förderinstrument überfrachtet, und besser an geeigneter Stelle geregelt wird. Es lässt sich hier festhalten, dass die Finanzierung der Weiterbildung durchaus den Unternehmen selbst überlassen werden kann. Etablierte Wissenschaftlerlnnen und TechnikerInnen bilden sich tendenziell anhand der konkreten, durchgeführten Projekte weiter und ergänzen ihre weiteren spezifischen Defizite durch Rekrutierungen oder Kooperationen. Eine Ausnahme stellt hier ev. die Umstellung von Arbeitsprozessen im Sinne von ,Industrie 4.0` dar. Die projektbegleitende Ausbildung von Nachwuchskräften ist jedenfalls eine vielversprechende Strategie, da dies eine praxisorientierte Finalisierung eines Ausbildungsabschnittes sicherstellt, und relativ oft in eine direkte Rekrutierung in beteiligte Unternehmen und Forschungseinheiten resultiert.

\section{Instrumentenwahl und -design}

Die grundsätzliche Logik dieses Branchenprogramms sollte sein, in enger Abstimmung mit der Branche (inkl. den Forschungseinheiten und der Luftverkehrswirtschaft) Schwerpunkte entlang von technologischen Roadmaps zu unterstützen, und damit durch eine längerfristige strategische Partnerschaft eine Risikoteilung zwischen der Industrie und dem Staat Österreich vorzunehmen, um mit Forschung und Entwicklung im Bereich der industriellen Forschung und experimentellen Entwicklung (TRL 2 bis 6) in diese Branche hineinzuwachsen bzw. seine Position zu festigen, deren Wertschöpfungsketten auf längerfristigen Verträgen basiert. Das Engagement der österreichischen Unternehmen in diesem Wachstumsmarkt scheint auch ungebrochen: Immerhin beinahe $70 \%$ der im Rahmen der Ö-LINK Studie befragten Unternehmen wollen ihre Bemühungen in der Luftfahrt in den nächsten 3-5 Jahren noch steigern (Brimatech 2015).

Die Wahl eines separaten thematischen Programms, das nicht-rückzahlbare Zuschüsse vergibt, ist einerseits anhand der Positionierung innerhalb des FuEPortfolios zu diskutieren, und andererseits anhand der Form der Unterstützung:

Die Form der Unterstützung als nicht-rückzahlbarer Zuschuss ist für Forschungsprojekte in den niedrigen Technology Readiness Levels (TRL 1/2-4/5, fallweise auch bis 
6) aufgrund der damit verbundenen hohen Risiken sicherlich vernünftig. Bei den höheren TRLs 5 und 6 bestehen konzeptionelle Überlappungen mit dem Basisprogramm mit seinem Portfolio an Finanzierungsunterstützung (siehe Darstellung im Anhang). Dies scheint auch insofern gerechtfertigt, als das Bündel an unterschiedlichen Risiken sehr projektabhängig ist, und die Langfristorientierung in der Luftfahrt insbesondere KMU vor Herausforderungen stellen kann. Damit ist eine gewisse Flexibilität der Handhabung von Vorteil, auch wenn prinzipiell anzuraten ist, dass Projekte auf den TRL-Stufen 5 und insb. 6 tendenziell eher dem Basisprogramm weiterzuverweisen wären. Dies ist auch in dem Kontext zu sehen, dass nach den unerfahrenen ,Neuzugängern' der letzten zehn Jahre nun mit einer zunehmenden Professionalisierung der KMU in der Branche zu rechnen ist.

Die Position von Take Off innerhalb des FuE-Portfolios lässt sich idealerweise mit kooperativer FuE in niedrigen TRLs (in der Regel TRL 2 bis 5, maximal bis TRL 6) beschreiben, die nationale und internationale Kooperationen anstoßen. In der Luftfahrt aktive Unternehmen engagieren sich auch relativ stark im Basisprogramm der FFG; der Unterschied der Projekte ist einerseits der vorwettbewerbliche Charakter der Forschung (d.h. kooperative vs. einzelbetriebliche Projekte) und andererseits der adressierte TRL.

Die Evaluierung zeigte, dass die Weiterentwicklung von vorhandenen Sicherheitsund Fluginformationssystemen eine höhere Dringlichkeit bzw. Marktfähigkeit aufweist als die weiteren Themenbereiche von Take Off. Dies drückt sich unter anderem auch dadurch aus, dass alle ATM-Projekte der Unternehmen nach Projektende weitergeführt wurden. Damit hat sich die mit der Verabschiedung der Österreichischen Luftfahrtstrategie einhergehende stärkere Öffnung des Programms für die Luftverkehrswirtschaft als sinnvoll erwiesen.

Ein Branchenprogramm wie Take Off bedarf eines aktiven Portfoliomanagements vor dem Hintergrund des breiten Angebots des Bundes und der Länder, das von der FFG im Bereich ihrer Möglichkeiten bereits bisher gut umgesetzt wurde. Ein Mehrwert für Unternehmen wird nicht nur durch die finanzielle Unterstützung geschaffen, zusätzlicher Nutzen wird auch durch inhaltliche Querverweise etc. gestiftet. Die Voraussetzung dafür - die bei TAKE OFF derzeit gegeben ist - ist ein Programmmanagement mit Erfahrung bzw. einem guten Überblick.

\section{Vernetzung bzw. Verbundenheit}

Die Ausrichtung von Take Off auf kooperative Projekte, begleitet von unterstützenden (letztens auch verpflichtend zu besuchenden) Vernetzungsaktivitäten, kann als gelungen angesehen werden. Sowohl Unternehmen als auch Forschungseinheiten reihen bei einer Frage nach der Unterstützung der Programmziele die „stärkere Vernetzung der österreichischen FuE-Community" als ein primär unterstütztes Ziel, das nicht zuletzt auf die recht zahlreichen Begleitmaßnahmen zurückzuführen sein dürf- 
ten. Entsprechend dem Instrumentendesign fühlen sich Unternehmen wie auch Forschungseinheiten als Folge der Partizipation an Take Off primär auf nationaler und europäischer Ebene stärker in FuE- und Innovationsnetzwerke integriert; speziell einige wissenschaftliche Akteure konnten sich mit den Take Off Projekten international positionieren. Neue Konsortien kamen nicht nur bei Vernetzungsveranstaltungen und als Folge von konkreten Ausschreibungen zustande, sondern wurden teilweise durch aktive Moderation von Prozessen geschaffen, wie zum Beispiel im Rahmen der Technologieplattform Offset.

Damit einher gehen auch Spillover-Effekte innerhalb der Luftfahrtzulieferindustrie und in benachbarte Branchen wie die Fahrzeugtechnologie. Aber es existieren auch weniger offensichtliche Querverbindungen mit z.B. dem Maschinenbau, Werkstoffteile und Sensortechnik, Bauwerküberwachung und Bahnverkehr. Der Technologietransfer zwischen den Branchen findet zu einem relativ hohen Ausmaß statt: In über der Hälfte der Fälle wird eine Technologie in anderen Unternehmen angewendet bzw. in rund einem Drittel der Fälle auch im Rahmen von „branchenfremden“ Verwendungen. Dies ist insbesondere als positiv zu beurteilen, als die großteils hochtechnologischen Lösungen der Luftfahrtindustrie mitunter aufgrund von hohen Kosten eine Barriere für die weitere Verbreitung darstellt.

Von den rund 240 österreichischen, im Bereich Luftfahrt aktiven Unternehmen im Jahr 2009 (Brimatech 2010) und rd, 215 Unternehmen im Jahr 2013 (Brimatech 2015) stehen rd. 55 Forschungseinheiten (inkl. einzelner Uni-Institute) gegenüber, die Teilaspekte der in der Luftfahrt nötigen technologischen Lösungen bearbeiten. Davon haben bislang 132 Unternehmen und praktisch alle Forschungseinheiten in Take Off Projekte durchgeführt. Damit liegt die „Marktdurchdringung“ auf Unternehmensseite bei rd. $60 \%$. Der Anteil von Erstfördernehmern hat sich von einem Niveau von rd. der Hälfte pro Ausschreibung auf rd. 25-30 \% (10-15 Unternehmen) in den Jahren 2012/13 verringert. Dies ist jedoch im Kontext mit immer höheren Zahlen von Erstantragstellern zu sehen, die aufgrund der wieder gesunkenen Budgets nicht bedient werden konnten. Ein Vergleich mit beantragten sonstigen Förderungen mit Hilfe der Ö-LINK-Daten zeigt, dass Unternehmen ohne Take Off Förderung durchaus auch anderwärtig aktiv werden (bei Mehrfachnennungen: 38 \% im Basisprogramm, $19 \%$ bei der AWS, und $23 \%$ beanspruchten regionale Förderungen - $54 \%$ erhielten keine Förderung). Damit scheint es trotz der bisherigen Erfolge zur Vernetzung der Luftfahrtzulieferindustrie noch Spielraum für weitere Aktivitäten zu geben.

\section{Effektivität, Wirkung und Effizienz}

Ein Programm ist effektiv, wenn es die gesetzten Ziele erreicht. Dies ist aufgrund von nicht präzise formulierten politischen Zielen oft nicht leicht nachzuvollziehen. Damit wird das Werteurteil der EvaluatorInnen relevanter, da dies über eine reine Zielerreichungsanalyse hinausgeht. 
Die oben beschriebenen Ziele von Take Off umfassen eine erhöhte Wettbewerbsfähigkeit, die Vernetzung, die Ausbildung, sowie die Unterstützung eines effizienten, sicheren, klimaschutz- und komfortorientierten Lufttransportsystems. Abgesehen von der unter „Relevanz und Kohärenz" geführten Diskussion können wir festhalten, dass unter dem Postulat der Wettbewerbsfähigkeit durchaus Effekte darstellbar sind.

Es ist eine höhere Inputadditionalität bei Projekten mit höheren Technology Readiness Levels (TRL) als Ausgangsbasis zu beobachten. Dies entspricht dem bekannten Phänomen, dass Unternehmen aufgrund des technologischen Risikos weniger gewillt sind eigene Mittel zu investieren, je näher ein Forschungsthema an der Grundlagenforschung liegt. Deshalb ist Take Off mit der Unterstützungsform eines reinen Zuschusses durchaus passend, solange Entwicklungen auf niedrigen TRLs (in der Regel TRL 2 bis 5, maximal bis TRL 6) unterstützt werden. Eine höhere Inputadditionalität ist aber z.B. im Basisprogramm zu erwarten.

Vor allem bei Projekten mit neuen Akteurskonstellationen ist teilweise eine sehr hohe Verhaltensadditionalität (Know-how Zuwachs aufgrund von thematischer Vertiefung, neue, strategische Kooperationen, etc.) zu beobachten. Dies spiegelt das Faktum wider, dass die Luftfahrtindustrie und -forschung in Österreich noch relativ jung ist, und während des letzten Jahrzehnts in relevante Themengebiete neu eindrang.

Insbesondere bei den Forschungsprojekten, die im Rahmen der Offset Technologieplattform initiiert wurden, war ein hoher Vorbereitungsaufwand für einzelne Projekte zu beobachten. Damit sind jedoch teilweise völlig neue Akteurskonstallationen entstanden, die für die beteiligten Unternehmen und Forschungseinrichtungen über das einzelne Projekt hinaus hohen Nutzen für die Folgeforschung bedeuteten.

Hinsichtlich der Output-Additionalität von Take Off Projekten können wir festhalten, dass die wirtschaftliche Verwertung von Projekten fallweise beachtliche Ausmaße annimmt: Obwohl im Rahmen der Befragung nur fünf von 34 Unternehmen angaben, dass sie bereits ökonomische Effekte realisieren konnten (dieser niedrige Wert entspricht der Grundannahme, dass ein hohes Risiko für die Unternehmen mit diesen Projekten verbunden ist und die Entwicklungen auch längerfristigen Charakter aufweisen), konnten diese fünf Unternehmen jedoch mit 82,8 Mio. Euro in Summe einen 8,5fach höheren ökonomischen Effekt für sich realisieren, als an Förderung an alle antwortenden Unternehmen ausgeschüttet wurde. Sie prognostizieren zudem weitere Effekte in Höhe von 47,9 Mio. Euro bis 2020, die in dieser Branche aufgrund der langfristigen Verträge auch realisierbar sein dürften.

Die Schätzung der gesamtwirtschaftlichen Wirkung aufgrund der Input-Output Analyse resultierte in einen Multiplikator der Bruttowertschöpfung von rd. 1:5, der dem Ergebnis in Deutschland doch recht nahe kommt. Das ist für eine kleine Volkswirtschaft wie Österreich mit dem einhergehenden höheren Anteil an Importen ein beachtliches Ergebnis. 
Abgesehen von der gesamtwirtschaftlichen Wirkung ist auch eine geringe, jedoch steigende Zahl an Unternehmen inzwischen strategisch besser positioniert; diese sind inzwischen im EU Rahmenprogramm, in internationalen Initiativen wie SESAR, Clean Sky, und auch ERA-Net AirTN aktiv. Insbesondere mit dem deutschen Luftfahrtprogramm LuFo konnte bereits im Rahmen des ERA-Net gut zusammengearbeitet werden. Hier werden österreichische Projektpartner inzwischen als gleichwertig angesehen, d.h. sie haben inzwischen einen wirklichen Mehrwert anzubieten und dies wird auch so wahrgenommen.

In einem Branchenprogramm wie Take Off, das zumindest für einzelne Projekte bewusst eine gewisse Durchlässigkeit hinsichtlich benachbarter Technologiefelder und Branchen zuließ, sind Technologietransfers in unterschiedliche Richtungen zwischen den Branchen und Technologiefeldern eher die Regel als die Ausnahme, und sind sehr zu begrüßen.

Der Beitrag zu einem „effizienten, sicheren, klimaschutz- und komfortorientierten Lufttransportsystem" ist primär aufgrund der Befragungsergebnisse sowie teilweise der Monitoringdaten $\mathrm{zu}$ beurteilen. Hierbei stellen die quantifizierten Ziele der VISION 2020 (ACARE) sowie Flightpath 2050 (EC) die Benchmarks dar, denen auch die österreichische Strategie sowie Take Off zuarbeiten (siehe Tabelle 1). Die durchaus herausfordernden Ziele können nach Clement und Billovits (2007, S. 20) „ohne technologischen Durchbruch nicht erreicht werden“. Gleichzeitig konnten wir jedoch in dieser Evaluation feststellen, dass die „Unterstützung eines klimaschutzorientierten Luftransportsystems“ zusammen mit der „komfortorientierten“ Zielstellung im Vergleich zu den anderen Programmzielen sowohl von den Unternehmen als auch den Forschungseinheiten in ihren Projekten eher als nachrangig eingestuft wird („nur" rd. $20 \%$ leisten einen "hohen Beitrag“). Dies steht in Dissonanz gegenüber dieser gesellschaftspolitischen Herausforderung. Das Thema Sicherheit spiegelt sich etwas stärker in den Zielsetzungen der einzelnen Take Off Projekte wider.

Das Kriterium der Effizienz setzt den Aufwand mit den erreichten Outputs eines Programms in Beziehung. Auch wenn die kooperativen Projekte mit rd. $€ 430.000$ /Projekt im Vergleich zu LUFO als recht bescheiden erscheinen, ist hier die großteils klein- bis mittelbetriebliche Struktur der österreichischen Luftfahrtzulieferindustrie mit zu bewerten. Das Gros der Fördermittel wurde dabei in den Jahren nach der Erstellung der FTI-Luftfahrtstrategie zur Verfügung gestellt. Nahezu drei Viertel der Förderbarwerte sind in der zweiten Hälfte des Beobachtungszeitraumes in Anspruch genommen worden. Hier ist zu betonen, dass eine gewisse Budgetsicherheit auf Jahre generell für Förderprogramme wichtig, und insbesondere als eine Voraussetzung für eine hohe KMU Beteiligung zu werten ist. 
Finanzielle und ökologische Nachhaltigkeit der Innovations- und FuEAktivitäten

Sowohl die Additionalität der Förderung als auch das teilweise hohe Risiko der Projekte kann dadurch festgemacht werden, als $55 \%$ der Projekte nach Förderende innerbetrieblich weiterverfolgt, und in höhere TRL Stufen bzw. der Vermarktung zugeführt wurden. Andererseits wurden $36 \%$ der Projekte (in technisch aufwändigen Bereichen) bislang nicht weiterverfolgt. Hierfür werden sehr unterschiedliche Gründe angeführt: Diese reichen von meist technologischem Entwicklungsrisiko bis hin zu fallweisen Interessensverschiebungen bei Unternehmen. Diese Rate ist gegeben den Programmzielen als durchaus entsprechend anzusehen.

Einerseits bedingt Umwelt und Nachhaltigkeit als gesellschaftspolitische Maxime eine Decarbonisierung des Luftverkehrs. Andererseits wünscht sich die Industrie aufgrund langfristig steigender Rohstoff- und Energiepreise Fluggeräte mit geringem Treibstoffverbrauch. Wie bereits oben argumentiert, sollte im Rahmen von Take Off verstärkt im Bereich des Luftfahrzeugbaus an Initiativen gearbeitet werden, welche den Treibstoffverbrauch weiter senken und die Lärm- und Umweltbelastung reduzieren. Dies wird wahrscheinlich ein etwas höheres Entwicklungsrisiko (auf niedrigen TRLs) nach sich ziehen, es wird jedoch den nationalen und internationalen FTILuftfahrtstrategien noch besser entsprochen.

\section{Handlungsempfehlungen}

HE 1: Die Luftfahrtindustrie ist durch weitere Konzentrationstendenzen gekennzeichnet. Die Systemhersteller haben die Integratorenrolle wahrzunehmen, daraus resultieren Konsolidierungsprozesse, und aufgrund eines Kostendrucks manchmal auch Produktionsverlagerungen. Da die OEM's Lieferanten reduzieren, wird die große Herausforderung der Zukunft darin bestehen die kritische Masse zu erreichen, damit die österreichischen Unternehmen nicht in die Grenzproduzentenrolle abgedrängt werden, die eher austauschbar sind.

Wir konnten feststellen, dass die Intention des Programmeigners, nämlich die spezifische Unterstützung der noch jungen Branche Luftfahrtzulieferindustrie mit einem eigenständigen Programm durchaus einen Beitrag liefern kann. Diese Branche hat sich seit der Etablierung von Take Off wesentlich weiterentwickelt, und scheint tw. auf einem guten Weg, um sich den anstehenden Herausforderungen stellen zu können.

Gegeben dem Durchdringungsstand der österreichischen Luftfahrzulieferindustrie mit Take Off (60\% der Unternehmen) und dem technologischen Entwicklungsstand so mancher Unternehmen, die sich noch nicht lange in der Luftfahrtindustrie engagieren, sollte das Programm für eine weitere Programmperiode verlängert werden. Nach dieser Periode sollte erneut überprüft werden, ob sich die Unternehmen 
bis dahin ausreichend in die Lieferketten integriert haben, und aufgrund dessen nach dieser nun folgenden Programmperiode aus den herkömmlichen FFG und EU Portfolios bedient werden sollten.

HE 2: Bei der Neuformulierung des Zielsystems für Take Off könnten die unterschiedlichen Ebenen, auf denen eine Wirkung erzielt werden soll, stärker berücksichtigt und voneinander getrennt werden. So kann bereits im Zielsystem unterschieden werden, welche sozio-ökonomischen Wirkungen in der österreichischen Luftfahrtbranche angestrebt werden (Impact), und welche Änderungen bei der Zielgruppe des Programms gewünscht werden (Outcome). Dies hätte zur Folge, dass die Gestaltung der Instrumente noch stärker als bereits jetzt auf das Zielsystem ausgerichtet erfolgen kann. Ebenso kann ein entsprechend formuliertes Zielsystem als Referenzrahmen für die Auswahl der Projekte dienen, so dass die Leistungen der Projekte in direktem Zusammenhang mit dem Zielsystem stehen. Weiters könnten alle Ziele durch eine weitere Konkretisierung gewinnen, d.h. qualitativ oder quantitativ präzisiert werden. Zum Beispiel sind als übergeordnete Vorgaben für Effizienz, die Klimaschutz- sowie Sicherheitsziele die Festlegungen in VISION 2020 und Flightpath 2050 zu nennen.

HE 3: Ein gesellschaftlich relevantes übergeordnetes Ziel von Take Off ist es, einen Beitrag zum Klimaschutz zu leisten. Da gerade der Luftverkehr zum einen besonders hohe Beiträge zu $\mathrm{CO}_{2}$ und $\mathrm{NO}_{x}$-Emissionen leistet, und zum anderen besonders lange Entwicklungszeiten hat, ist bei weiterhin steigendem Luftverkehrsaufkommen dieses Ziel in einen Forschungs- und Technologieprogramm von besonderer Bedeutung. Dieser Bedeutung werden die geförderten Projekte jedoch nur zum Teil gerecht (ca. 20 \% leisten einen sehr hohen Beitrag zur Reduktion von Emissionen). Auch in ihrer eigenen Wahrnehmung wird von den Projektwerbern kein hoher Beitrag zum Klimaschutz geleistet, jedenfalls weniger hoch als die Beiträge zum Erreichen der anderen Programmziele. Bei der Neugestaltung eines Folgeprogramms, sollten daher geeignete Wege gefunden werden, dem Klimaschutz einen höheren Stellenwert einzuräumen. Dies könnte beispielsweise durch eine höhere Gewichtung dieses Aspekts bei der Auswahl von Projekten geschehen. Auch durch die Etablierung eines speziellen Förderschwerpunkts „Umwelt- und Klimaschutz" könnte deutlich gemacht werden, dass hier ein besonderer Förderbedarf gesehen wird.

HE 4: Eine Komfortorientierung des Luftverkehrs stellt selbst aus der Perspektive der Programmteilnehmerinnen und -teilnehmer kein für sich genommen zu rechtfertigendes Ziel öffentlicher Förderung dar. Diese Ergänzung im Zielsystem könnte daher in Zukunft entfallen.

HE 5: Aus ordnungspolitischen Erwägungen sind geförderte Projekte sinnvoll, die einem hohem Risiko unterliegen und daher von Unternehmen ohne öffentliche Förderung nicht durchgeführt würden. Damit FuE einen volkswirtschaftlichen Nutzen stiften kann (Aufrechterhalten der Innovationskraft einer Branche, Technologietrans- 
fer in andere Bereiche), ist es sinnvoll, in solchen Fällen öffentliche FuE-Programme durchzuführen. Das Risiko für das Einzelunternehmen ist in der Regel umso höher, je niedriger der Technologiereifegrad ist, da zu frühen Zeitpunkten der Technologieentwicklung weniger gut abschätzbar ist, ob sich marktfähige Produkte oder Dienstleistungen daraus entwickeln lassen. Öffentliche Förderung sollte daher in der Regel auf niedrige TRL als Startpunkt abzielen. Auch wenn im Einzelfall Projekte sinnvoll sein mögen, die auf eine Weiterentwicklung einer Technologie bis hin zur Marktreife zielt, weil z.B. in der Luftfahrt besonders hohe Kosten mit der erfolgreichen Demonstration einer neuen Technologie verbunden sein können, sollte die Förderung höherer TRL innerhalb von Take Off eher die begründete Ausnahme bleiben. Take Off sollte tendenziell an der Grundregel festhalten, Vorhaben von einem TRL unter der Stufe 6 zu fördern, und darüber hinaus tendenziell an das Basisprogramm (mit niedrigeren Barwerten und einem Finanzierungsmix aus Zuschuss/Darlehen/Haftung) weiterverweisen.

HE 6: Schwerpunkt der Unterstützung der projektbegleitenden Ausbildung von Nachwuchskräften, und Weiterbildung von etablierten Mitarbeitern nur hinsichtlich der Weiterentwicklung von Arbeitsprozessen zur, Industrie 4.0‘. In Take Off wurde anfänglich die Weiterbildung von ForscherInnen und TechnikerInnen (mit abnehmender Tendenz) betrieben. Es lässt sich hier festhalten, dass die Finanzierung der Weiterbildung tendenziell den Unternehmen selbst überlassen werden kann. Die Weiterbildung von etablierten Mitarbeitern erfolgt hauptsächlich anhand konkreter FuE Projekte und weitere Defizite werden oft durch Kooperationen auszugleichen versucht. Allenfalls sind vereinzelt Ausbildungsformate unter dem Schlagwort Industrie 4.0 auf breiterer Basis gerechtfertigt. Die projektbegleitende Ausbildung von Nachwuchskräften ist hingegen eine vielversprechende Strategie, da dies eine praxisorientierte Finalisierung eines Ausbildungsabschnittes sicherstellt, und erfahrungsgemäß relativ oft in eine direkte Rekrutierung in beteiligte Unternehmen resultiert. Dies scheint für die spezifischen technologischen Anforderungen der Luftfahrtindustrie besonders geeignet und könnte in Zukunft ausgeweitet werden. Hier sind Formate auf unterschiedlichen formalen Qualifikationsniveaus denkbar, und wären am besten nach Konsultation mit den Unternehmen festzulegen.

HE 7: Die Veröffentlichung der Projektergebnisse in den Fachmedien intensivieren. Von einer höheren Anzahl von Veröffentlichungen profitiert in erster Linie die FuE-Community der österreichischen Luftfahrtindustrie und -forschung. Aber auch über die Luftfahrtbranche hinaus wird ein Technologie-Spillover nur erleichtert, wenn die Ergebnisse der Forschungs- und Technologieprojekte zugänglich sind. Bei einzelnen Projekten mit höheren TRL kann es aufgrund des hohen Wettbewerbsdrucks für die Unternehmen wichtig sein, das geistige Eigentum schützen zu können. Ausnahmen in einzelnen, begründeten Fällen sollten daher zulässig sein.

HE 8: Wir konnten feststellen, dass die Intention des Programmeigners, nämlich die spezifische Unterstützung der noch jungen „Branche“ Luftfahrtzulieferindustrie mit 
einem eigenständigen Programm durchaus ein aktives Portfoliomanagement benötigt, da Interaktionen und Überlappungsbereiche mit anderen Programmen gegeben sind. In diesem Zusammenhang stellt sich auch die Frage, ob Take Off als eigenständiges Programm weiter bestehen, oder im inhaltlich naheliegenden bmvit Schwerpunkt ,Mobilität der Zukunft' untergeordnet werden soll. Hier sind die Für und Wider abzuwägen. Einerseits ist Take Off inzwischen eine etablierte Marke. Da eine zielgruppenspezifische Ansprache von Unternehmen bei der Vielzahl an Programmen heutzutage wichtiger ist als je zuvor, sollte diese Marke bei einer Weiterführung des Programms nicht gefährdet werden. Andererseits wurde Take Off bislang alleine als ein Branchenförderinstrument implementiert, und gesellschaftspolitischen Belangen wie Umweltschutz, Multimodalität, etc. wurde tendenziell eine geringere Rolle zugeschrieben, die jedoch in Mobilität der Zukunft eine wichtige Rolle spielen. Der linearen Vision des „immer größer und besser" stehen z.B. auch andere Visionen gegenüber, in denen flexiblere bzw. kleinere Strukturen und Flugzeuge integrativ mit anderen Verkehrsträgern (bei gleichem Komfort) multimodale Formen der Fortbewegung erleichtern. Wir empfehlen eine interne Reflexion hinsichtlich dieses Themenbereichs. Die angesprochenen Schwerpunktsetzungen sowie die administrative Organisation innerhalb des Ministeriums wird eine Entscheidung pro oder contra einer Eigenständigkeit von Take Off bedingen.

HE 9: Die Vernetzung mit internationalen Foren und Programmen nimmt in einem derart langfrist-orientierten und strategischen Markt einen wesentlichen Teil des Programmmanagements ein, das de facto von bmvit und FFG zusammen verfolgt wird. Hierbei hat das Vorhandensein eines eigenen Programms die Teilnahme an ERA-Net erleichtert, das in Zukunft noch nach Zielmärkten ausgebaut werden kann. so wurden z.B. gemeinsame Ausschreibungen insb. mit Deutschland durchgeführt, und daraufhin die nationalen Teile mit den jeweiligen thematischen Programmen (hier: Take Off und LuFo) unterstützt. Dies spricht für eine Beibehaltung des Programms, da die spezifischen Ausschreibungen eine Koordination mit anderen Förderprogrammen erleichtert.

HE 10: Es sei an dieser Stelle noch einmal auf den bekannten Umstand hingewiesen, dass planbare Förderbudgets auf Jahre als die Voraussetzung für eine Verstetigung von Forschungsbemühungen, insbesondere in $\mathrm{KMU}$, verstanden werden kann. Im Vergleich zu den Anfangsjahren von Take off konnte die budgetäre Situation ab 2008 zwar tendenziell verbessert werden, es lässt sich dennoch keine mittelfristige Regelmäßigkeit, und damit Planbarkeit, erkennen. 


\section{Anhang}

\subsection{Abdeckung der Technologiereifegrade}

\section{Grafik 36 Förderungen entlang der Technologiereifegrade}

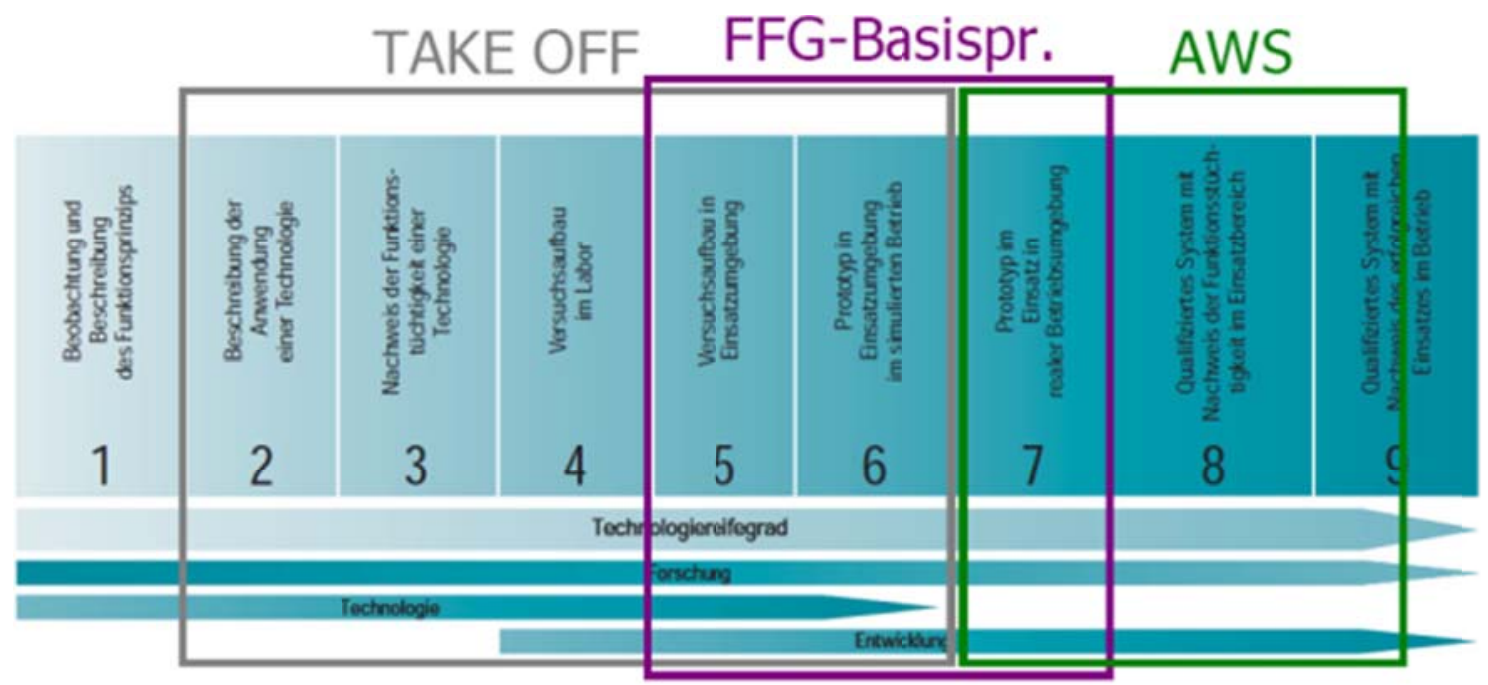

Quelle: bmvit Eigendarstellung

\subsection{Literatur}

ACARE (2002). Strategic Research Agenda. Executive Summary, October 2002. http://www.acare4europe.com/sites/acare4europe.org/files/document/executivesummary.pdf, Zugriff am 08.08.2014

ACARE (2004). Strategic Research Agenda. Executive Summary, October 2004. http://www.acare4europe.com/sites/acare4europe.org/files/document/ASDexec \%20sum-2nd-final-171104-out-asd.pdf, Zugriff am 08.08.2014 ACARE (2008). http://www.acare4europe.com/sites/acare4europe.org/files/document/ACARE 2008 Addendum.pdf, Zugriff am 08.08.2014

ACARE (2012). Realising Europe's vision for aviation. Strategic Research \& Innovation Agenda, Volume 1, http://www.acare4europe.org/sites/acare4europe.org/ files/attachment/SRIA \%20Volume \%201.pdf, Zugriff am 08.08.2014 
ACARE (2012). Realising Europe's vision for aviation. Strategic Research \& Innovation Agenda, Volume 2, http://www.acare4europe.org/sites/acare4europe.org/ files/attachment/SRIA \%20Volume \%202.pdf, Zugriff am 08.08.2014

BMVIT (2008). FTI-Luftfahrtstrategie Österreichische Forschungs-, Technologie- und Innovationsstrategie für die Luftfahrt. http://www.bmvit.gv.at/innovation/ downloads/luftfahrtstrategie.pdf, Zugriff am 08.08.2014

BMWi (2014). Die Luftfahrtstrategie der Bundesregierung, Stand März 2014. http://www.bmwi.de/BMWi/Redaktion/PDF/J-L/luftfahrtstrategie-derbundesregierung, property=pdf,bereich=bmwi2012,sprache=de,rwb=true.pdf, Zugriff am 08.08.2014

Bräuninger, M.; Döll, S.; Nolte, A.; Wohlers, E. (2010): Zukunftsperspektiven der Luftfahrtindustrie - Chancen und Risiken für das Luftfahrtcluster in der Metropolregion Hamburg, Studie im Auftrag der HSH Nordbank, Hamburg.

Brimatech (2010). Österreichische Luftfahrtindustrie: Datenbank der Marktteilnehmer (Ö-LINK). Studie finanziert durch das Bundesministerium für Verkehr, Innovation und Technologie, Wien

Brimatech (2015). Ö-LINKupdate - Erhebung zur Aktualisierung der Luftfahrtdatenbank Ö-LINK. Studie finanziert durch das Bundesministerium für Verkehr, Innovation und Technologie, Wien

Clement, W., Billovits, A. (2007). FTI-Aeronautik-Strategie für Österreich: Internationale Aspekte und ökonomische Rahmenbedingungen. Studie im Auftrag des Bundesministerium für Verkehr, Innovation und Technologie, Wien

Drechsler, K., Hainisch, J., Ladstätter, E. et al. (2011). Aus- und Weiterbildung in der österreichischen Luftfahrtindustrie: Ansatzpunkte zur Bedarfsdeckung. Eine Studie finanziert im Rahmen der 7. Ausschreibung des Forschungs- und Technologieprogramms für die Luftfahrt Take Off, Wien.

European Aeronautics, A Vision for 2020. Meeting society's needs and winning global leadership. Report of the Group of Personalities, January 2001. http://ec.europa.eu/research/growth/aeronautics2020/pdf/aeronautics2020 en.pdf, Zugriff am 08.08.2014

European Commission (2011). Flightpath 2050, Europe's Vision for Aviation. Report of the High-Level Group on Aviation Research.

http://ec.europa.eu/research/transport/pdf/flightpath2050 final.pdf, Zugriff am 08.08.2014

European Commission (2013). Increasing the sustainability of air transport, http://www.transport-research.info/Upload/Documents/201310/20131031 19495480523 PB06 WEB.pdf, Zugriff am 08.08.2014 
FFG (2007). 6. EU-Rahmenprogramm für Forschung, technologische Entwicklung und Demonstration. http://rp7.ffg.at/upload/medialibrary/Taetigkeitsbericht RP6.pdf, Zugriff: 14.08.2014

Forstinger M., Romeike F., Pöchhacker-Tröscher, G. (2014). Risikomanagement in der österreichischen Luftfahrtindustrie - eine internationale Benchmark-Studie. Im Auftrag des Bundesministerium für Verkehr, Innovation und Technologie, Wien Groß, W., Kerlen, C. et al. (2013). Das Luftfahrtforschungsprogramm des Bundesministeriums für Wirtschaft und Technologie - Ergebnisse der Evaluation 2012. Zusammenfassung und Gestaltungsempfehlungen

Knoerzer, D. (2012). Towards the Future of Aviation. Aeronautics and Air Transport Research of the European Union. http://www.wakenet.eu/fileadmin/user_upload/ 4th_major_WN3E-Workshop/presentations/WN3E_1_01b_Knoerzer \%204th \%20Workshop \%20WakeNet3 \%20Final \%20Langen \%2028-02-2012.pdf; Zugriff: 08.08.2014

Kumar, N. (2013). Strategic Research and Innovation Agenda (SRIA) - response to Flightpath 2050. http://www.airtn.eu/downloads/acare.pdf, Zugriff am 08.08.2014 Miller, R. E., Blair, P. D. (2009), Input-Output Analysis - Foundations and Extensions. Cambridge University Press, New York.

Oltmanns, E., Bolleyer, R., Schulz, I. (2009). Forschung und Entwicklung nach Konzepten der Volkswirtschaftlichen Gesamtrechnung. In: Wirtschaft und Statistik, Statistisches Bundesamt, 2/2009, S.125-135.

Pöchhacker-Töscher, G., Negeli-Ganz, B., Hainisch, J. (2010a). Position österreichischer Luftfahrt-Unternehmen in der Zulieferkette und Überlegungen zu clusterpolitischen Maßnahmen in Österreich, Studie im Auftrag des Bundesministerium für Verkehr, Innovation und Technologie, Wien

Pöchhacker-Töscher, G., Negeli-Ganz, B., Hainisch, J., Schmidt-Bischoffshausen, H., Gradinger, R. (2010b). Erhebung der Innovations- und Kooperationspotenziale in ausgewählten Segmenten der österreichischen Luftfahrtindustrie, Studie im Auftrag des Bundesministerium für Verkehr, Innovation und Technologie, Wien PROVISO Bericht Juni 2014. 7. EU-Rahmenprogramm für Forschung, technologische Entwicklung und Demonstration (2007-2013). Stand der Daten 12/2013, Wien Statistik Austria (2014). Input-Output-Tabelle 2010, inklusive Aufkommens und Verwendungstabelle. Wien 


\subsection{Interviews}

\section{InterviewpartnerInnen}

bmvit: Mag. Evelinde Grassegger, Mag. Ingolf Schädler, Mag. Michael Wiesmüller, DI Ingrid Kernstock, Mag. Elisabeth Osberger; FFG: DI Vera Eichberger; Nationale und internationale Expert/innen: Gerben Klein Lebbink (Coordinator Aeronautics International - AgentschapNL), Friedrich König (Leitung von LuFo bei DLR), Mag. Johanna Egger-Berndorfer (Brimatech), Wolfram Anderle (Leitung des aws Geschäftsfelds Technologie)

\section{Interviewleitfaden}

1. Skizzieren Sie bitte die Entstehungsgeschichte und Entwicklung bis dato. Mission, Ziele, organisatorische Strukturen (bmvit u. FFG).

a. Neuausrichtung 2007: (anhand der 3 Folien durchgehen) Maßnahmen (bspw: Leitprojekte).

b. Wichtigste Einflussfaktoren für die Entwicklung? Politische Einflussfaktoren?

c. Haupterfolge? Probleme? (Nischenführerschaft / Stärkung der Internationalität)

2. Wie wurde der Schritt von thematischen Schwerpunkten zu Marktsegmenten gemacht? Passt noch?

3. Rolle der Technologieplattform Offset

4. Abgrenzung von Take Off (Bauteile) zu nationalen Programmen: BP/PdZ (Verfahren), BRIDGE, Headquarters, COMET u.a. im Programmdokument 2013 beschrieben.

a. Rolle von Schnittstellen: innerhalb der FFG, zu AWS. Wo gibt es Adaptionsbedarf?

5. Funktion von Begleitmaßnahmen vs. technologischen Förderprojekten.

a. Begleitmaßnahmen (17/Jahr 2019 und 11/Jahr 2010!): Def.: Generierung von Know-how; Transfer; Transfer von anderen Forschungssträngen hin zur Luftfahrt - und vice versa.

b. Förderungen im Bereich Humanressourcen

c. Anteil an vorwettbewerblicher Forschung. - Umgang damit?

6. Prozess der Ausschreibung(svorbereitung), Projektauswahl, -begleitung, und Diffusion:

a. Wie ist der Prozess, um zu neuen Ausschreibungsschwerpunkten zu gelangen? 
b. Wie werden Querschnittsthemen wie Umwelt und Gender berücksichtigt?

c. Inkludierung von ausländischen Partnern; Unterstützung bei Konsortialverträgen

\section{International:}

7. Einbettung/Abgrenzung von Take Off zu internationalen Programmen:

a. Einfluss der Strategie durch Luftfahrttechnologieplattform ACARE sowie des Weißbuchs der EC

b. Wie ist die Teilnahme an den Leitprojekten SESAR und JTI Clean Sky bzW. anderen Demonstratorprojekten bislang verlaufen? In welche Richtung wird sich das entwickeln?

c. Wie in FP7 (60\% Rücklaufquote angestrebt) -> Wo stehen wir heute?

d. Wie in ERA-net AirTN / welche national (Wertschöpfungsketten, Themen)

e. Teilnahme am ERA-NET AirTN inkl. gemeinsamer Ausschreibungen. Rolle von Schnittstellen: Adaptionsbedarf? Koordination mit benachbarten Ländern auf bilateraler Ebene noch nötig? EUREKA?

8. Take off Sub-Ziel laut Eval-Ausschreibung: Impulsmaßnahmen zum Aufbau von Kooperationsmöglichkeiten mit Amerika und Asien?

\section{Strategie:}

9. In welchen Aspekten ist die Luftfahrtindustrie und -forschung derzeit noch fragmentiert?

10. Vision 2020: Organisatorische Maßnahmen, Weiterentwicklung Förderung \& Finanzierung; Ausbildung und Qualifikationsmaßnahmen, Begleitmaßnahmen (Schaubild: Luftfahrtstrategie und Maßnahmen)

11. Was spricht dafür/dagegen Take Off als separates Programm beizubehalten, was spricht dafür/dagegen es in MdZ zu integrieren? Was hat Take Off geschafft, was es als Teil von MdZ nicht geschafft hätte?

12. Ausschreibung Herbst 2014 - wieso hat sich die Neuauflage des Programms hinausgezögert?

13. Direkte Wirkungen. Indirekte Wirkungen. Wo sehen Sie die Hebelwirkung des Programms?

14. Themenschwerpunkte in Zukunft: Soll es eine Fokussierung geben oder generelle Strategie wie gehabt? Abhängigkeit von zu erwartenden Budgets. 
KMU Forschung Austria

Austrian Institute for SME Research 